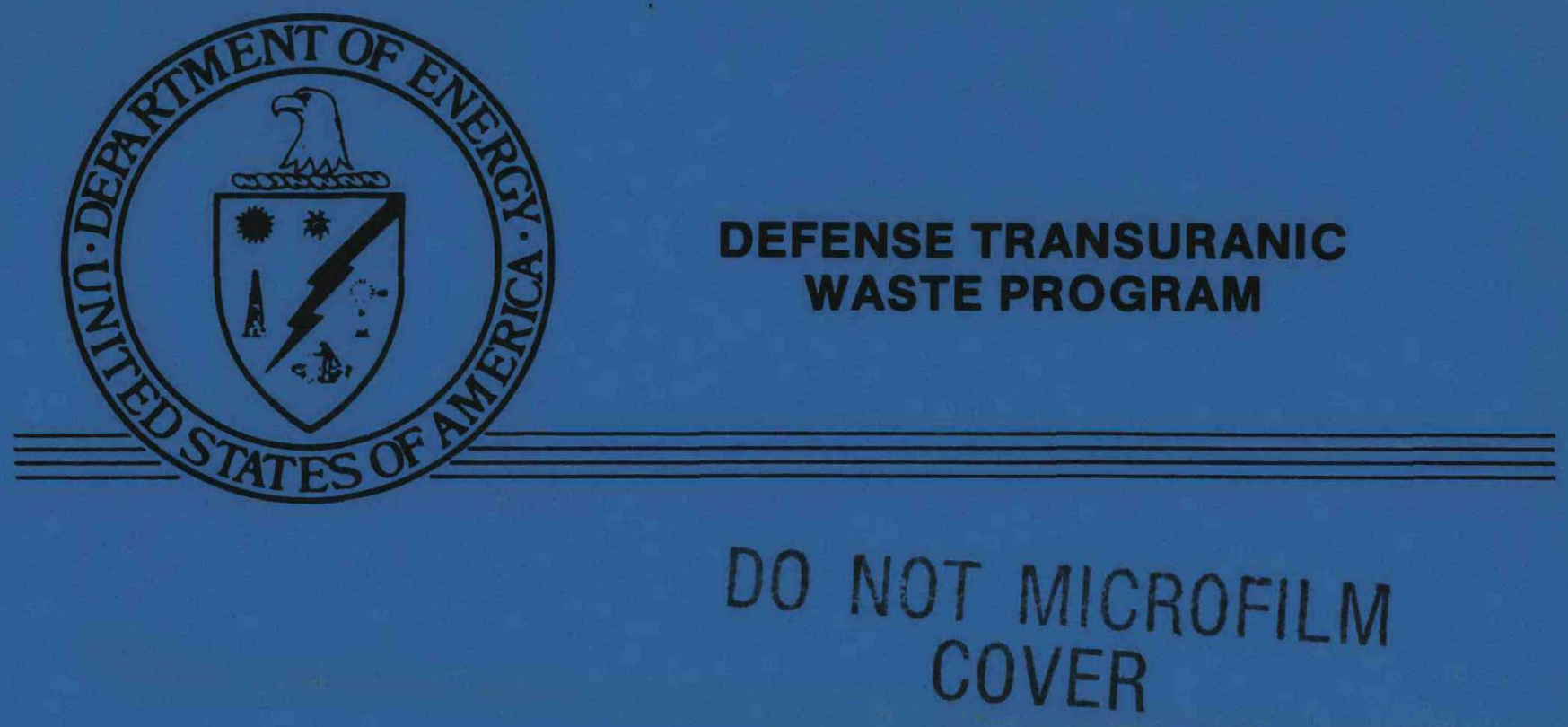

\title{
CHARACTERISTICS OF TRANSURANIC WASTE AT DEPARTMENT OF ENERGY SITES
}

May 1983 


\section{DISCLAIMER}

This report was prepared as an account of work sponsored by an agency of the United States Government. Neither the United States Government nor any agency Thereof, nor any of their employees, makes any warranty, express or implied, or assumes any legal liability or responsibility for the accuracy, completeness, or usefulness of any information, apparatus, product, or process disclosed, or represents that its use would not infringe privately owned rights. Reference herein to any specific commercial product, process, or service by trade name, trademark, manufacturer, or otherwise does not necessarily constitute or imply its endorsement, recommendation, or favoring by the United States Government or any agency thereof. The views and opinions of authors expressed herein do not necessarily state or reflect those of the United States Government or any agency thereof. 


\section{DISCLAIMER}

Portions of this document may be illegible in electronic image products. Images are produced from the best available original document. 


\section{DISCLAIMER}

This report was prepared as an account of work sponsored by an agency of the United States Government. Neither the United States Government nor any agency thereof, nor any of their employees, makes any warranty, express or implied, or assumes any legal liability or responsibility for the accuracy, completeness, or usefulness of any information, apparatus, product, or process disclosed, or represents that its use would not infringe privately owned rights. Reference herein to any specific commercial product, process, or service by trade name, trademark, manufacturer, or otherwise, does not necessarily constitute or imply its endorsement, recommendation, or favoring by the United States Government or any agency thereof. The views and opinions of authors expressed herein do not necessarily state or reflect those of the United States Government or any agency thereof.

\section{DO NOT MICROFILM COVER}




\title{
CHARACTERISTICS OF TRANSURANIC WASTE AT DEPARTMENT OF ENERGY SITES
}

\author{
R. T. Jensen \\ F. J. Wilkinson III \\ Rockwe 11 International \\ Energy Systems Group \\ Rocky Flats Plant \\ Golden, Colorado 80401
}

May 1983

NOTICE

PORTIONS OF THIS REPORT ARE ILLEGIBLE.

It has been reproduced from the best available copy to permit the broadest possible availability.

\author{
Prepared for \\ U. S. Department of Energy \\ Under Contract DE-ACO4-76DP03533
}

\section{DISCLAIMER}

\begin{abstract}
This report was prepared as an account of work sponsored by an agency of the United States Government. Neither the United States Government nor any agency thereof, nor any of their employees, makes any warranty, express or implied, or assumes any legal liability or responsibility for the accuracy, completeness, or usefulness of any information, apparatus, product, or process disclosed, or represents that its use would not infringe privately owned rights. Reference herein to any specific commercial product, process, or service by trade name, trademark, manufacturer, or otherwise does not necessarily constitute or imply its endorsement, recommendation, or favoring by the United States Government or any agency thereof. The views and opinions of authors expressed herein do not necessarily state or reflect those of the United States Government or any agency thereof.
\end{abstract}

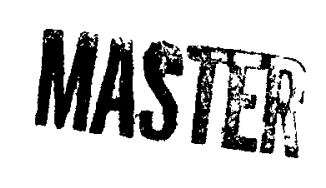




\section{ACKNOWLEDGMENT}

The authors acknowledge the Department of Energy (DOE) and the Rockwell International Transuranic Waste Systems Offices (TWSO) for providing the funding and guidance for this work. L. J. Smith of TWSO has been instrumental in providing help on the format and references. S. S. Martin of the Rocky flats Plant (RFP) did a large part of the data acquisition. J. G. Warren of the LoS Alamos National Laboratory (LANL), H. M. Batchelder of Idaho National Engineering Laboratory (INEL), J. D. Anderson and B. M. Poremba of Rockwell Hanford Operations (RHO), L. M. Cheevers of Argonne National Laboratory-East, D. Gillas of Reynolds Electrical \& Engineering Company, R. K. Blauvelt of Monsanto Research Corporation, E. M. King of Oak Ridge National Laboratory (ORNL), and C. Stevens of Savannah River Plant (SRP) provided a large part of the data used in this report. Their time and cooperation in assembling the data was invaluable. We also thank G. A. Riordan for the technical editing of the manuscript, his efforts are appreciated. 


\section{FOREWORD}

Transuranic (TRU) waste as defined by the DOE is material that, without regard to source or form at the end of institutional control periods, is contaminated with alpha-emitting radionuclides of atomic number greater than 92 and has a half-life greater than 20 years and a concentration greater than 100 nanocuries per gram. However, the information in this report has been collected under the old definition that states that the waste need only be of long half-life and concentration greater than 10 nanocuries per gram alpha activity. The older definition also includes all TRU nuclides except ${ }^{238} \mathrm{Pu}$ and ${ }^{241} \mathrm{Pu}$, and in addition, includes the ${ }^{233} \mathrm{U}$ isotope and its daughter products.

Before 1970, all TRU waste was buried in pits and trenches. In March of 1970, the U. S. Atomic Energy Commission (AEC), a predecessor of the DOE, instituted a policy requiring that waste known or suspected of containing significant quantities of TRU radionuclides be segregated from other types of radioactive waste. Such waste was to be packaged and stored so as to be retrievable, in containers free of external contamination, for a period of at least 20 years, pending the development of a suitable federal repository. ${ }^{2}$ In 1973, 10 nanocuries per gram $(\mathrm{nC} i / g)$ of alpha activity was established as a threshold at which waste would be defined as TRU waste. In this report, waste that was packaged and set aside is referred to as "stored" waste. Waste that was buried prior to this period of segregation is called "buried" waste. Finally, waste that is currently being produced at DOE sites is called "newly generated" waste. This report is organized using those three categories.

To implement a long-term strategy for handling TRU waste, the DOE established a lead office for the Defense Transuranic Waste Program. It consists of the Albuquerque Operations office (ALO) of the DOE which 
manages the program, and Rockwell International - Rocky Flats Plant (RI-RFP), as the lead support contractor. The TRU waste inventories and projections in this study were compiled to provide data for planning and analysis activities of the lead office. Most of the data were obtained from waste management offices or data management centers at DDE sites. 
ACKNOWLEDGMENT $\ldots \ldots \ldots \ldots \ldots \ldots \ldots \ldots \ldots \ldots \ldots \ldots \ldots \ldots \ldots \ldots \ldots \ldots$

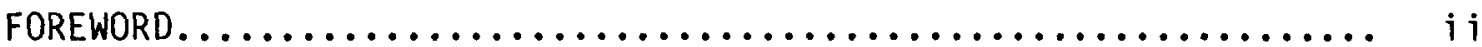

CONTENTS...................................... iv

TABLES $\ldots \ldots \ldots \ldots \ldots \ldots \ldots \ldots \ldots \ldots \ldots \ldots \ldots \ldots \ldots \ldots \ldots \ldots \ldots \ldots \ldots \ldots$

FIGURES $\ldots \ldots \ldots \ldots \ldots \ldots \ldots \ldots \ldots \ldots \ldots \ldots \ldots \ldots \ldots \ldots \ldots \ldots \ldots \ldots \ldots \ldots \ldots$

QUICK LOCATOR GUIDE............................. xi

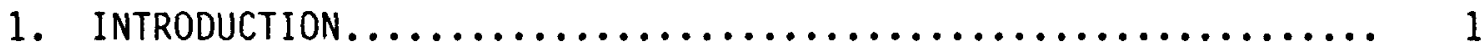

2. CHARACTERISTICS OF NEWLY GENERATED TRU WASTE............ 7

2.1 Contact-Handled - Summary.................... 7

2.1.1 Locations, Quantities, and Waste Forms......... 7

2.1.2 Activities that Generate TRU Waste........... 11

2.1.3 TRU Content........................... 11

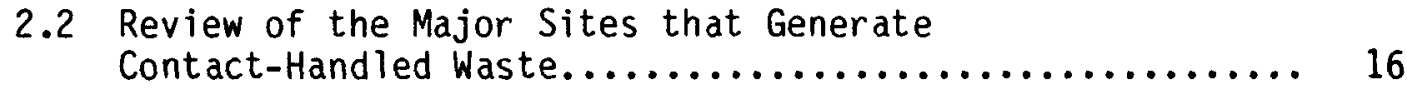

2.3 Remote-Handled - Summary..................... 28

2.3.1 Locations, Quantities, and Waste Forms......... 28

2.4 Review of the Major Sites that Generate

Remote-Handled Waste........................ 32

3. CHARACTERISTICS OF STORED TRU WASTE................ 38

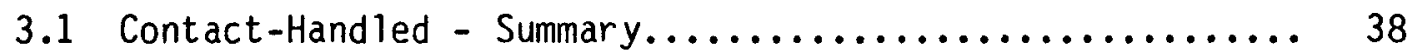

3.1.1 Locations, Quantities, and Waste Forms......... 38

3.1.2 Transuranic Content and Surface Dose Rate....... 42

3.2 Review of $\mathrm{CH}$ Waste Packages at Storage Sites.......... 48

3.3 Remote-Handled - Summary...................... 91

3.4 Review of the RH Waste Categories at Storage Sites...... 91 
4. CHARACTERISTICS OF BURIED TRU WASTE................ 104

5. ADDITIONAL SOURCES OF TRU WASTE INFORMATION............ 110

5.1 Further Reports on Subjects Not Covered

In This Document............................ 110

5.2 Information Available at Individual Sites........... 111

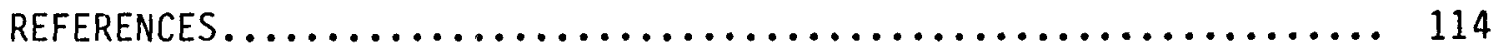

APPENDIX A.- Glossary of Abbreviations and Acronyms............ 118

APPENDIX B.- Examples of Tables Compiled from Computer.......... 119 Data at INEL, Los Al amos, Hanford, and Savannah River

APPENDIX C.- Storage Site Environmental Impact Reviews.......... 144 
TABLES

Page

1.2 Major Classifications of TRU Waste................ 3

2.1 Projection of Contact Handled TRU Waste - Volumes and Waste Forms............................. 10

2.4 Activities That Generate TRU Waste.................. 12 (Cubic Meters Per Year 1981-1985)

2.5 Forecast of Major TRU Isotopes Contained in Newly Generated Waste (Average Per Year)................... 14

2.6 Typical Isotopic Composition of Weapon Plutonium/ Typical Isotopic Composition of Heat Source Plutonium.......

2.7 Forecast of Transuranic Waste Generation at Argonne National Laboratory - East (Contact-Handled).

2.8 Forecast of Transuranic Waste Generation at Battelle-PNL (Contact handled)

2.9 Forecast of Transuranic Waste Generation at Los Alamos

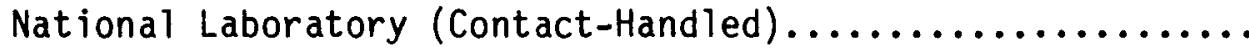

2.10 Forecast of Transuranic Waste Generation at Lawrence Livermore National Laboratory (Contact-Handled).............

2.11 Forecast of Transuranic Waste Generation at Mound Facility (Contact-Handled).

2.12 Forecast of Transuranic Waste Generation at Oak Ridge

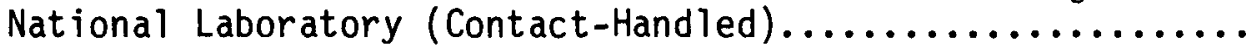

2.13 Forecast of Transuranic Waste Generation at Rocky

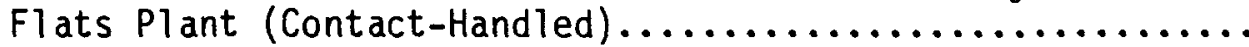

2.14 Forecast of Transuranic Waste Generation at Rockwell

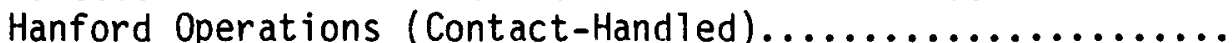

2.15 Forecast of Transuranic Waste Generation at Savannah River Plant and Laboratory (Contact-Handled).

2.16 Forecast of Transuranic Waste Generation at Westinghouse Hanford Company (Contact-Handled). 
2.17 Forecast of Transuranic Waste Generation at Sites Generating Less Than $25 \mathrm{~m}^{3} / \mathrm{yr}$ (Contact-Handled)...........

2.18 Projection of Remote Handled TRU Waste Volumes and waste Forms................................. 29

2.19 Forecast of Transuranic Waste Generation at Argonne National Laboratory - East (Remote-Handled).

2.20 Forecast of Transuranic Waste Generation at Idaho National Engineering Laboratory (Remote-Handled)......... 34

2.21 Forecast of Transuranic Waste Generation at Los Alamos National Laboratory (Remote-Handled).......... 35

2.22 Forecast of Transuranic Waste Generation at Oak Ridge National Laboratory (Remote-Handled).......... 36

2.23 Forecast of Transuranic Waste Generation at Westinghouse Hanford Company (Remote-Handled)........... 37

3.1 Contact Handled Waste Forms at TRU Storage Sites (Vo1.\%).... 39

3.4 Effect of Container Volume on Waste Form Volume.......... 43

3.5 Major TRU Radionuclide Content in Stored Wastes (To December 31, 1980).

3.6 Estimated TRU Isotopes in Buried and Stored Waste........ 46

3.10 Contact-Handled Categories at TRU Waste Storage Sites (To December 31, 1980).

3.11 Detailed Data on Contact-Handled TRU Waste Storage

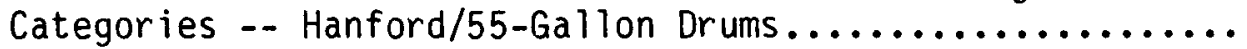

3.12 Detailed Data on Contact-Handled TRU Waste Storage

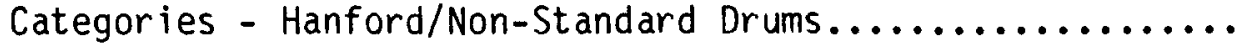

3.13 Detailed Data on Contact-Handled TRU Waste Storage

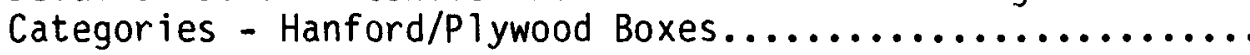

3.14 Detailed Data on Contact-Handled TRU Waste Storage

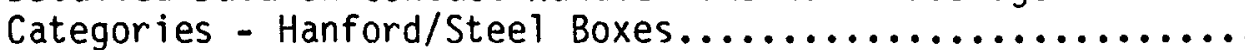

3.15 Detailed Data on Contact-Handled TRU Waste Storage Categories - INEL/55-Gallon Drums.................. 58

3.16 Content Data for $55-G a l l o n$ Drums at INEL.............. 59 
3.18 Detailed Data on Contact-Handled TRU Waste Storage Categories - INEL/30-Gallon Drums.................... 61

3.18 Detailed Data on Contact-Handled TRU Waste Storage Categories - INEL/83-Gallon Drums..................... 62

3.19 Detailed Data on Contact-Handled TRU Waste Storage Categories - INEL/Drums in Cargo Containers and Bins........

3.20 Detailed Data on Contact-Handled TRU Waste Storage Categories - INEL/Non-St andard Drums.

3.21 Detailed Data on Contact-Handled TRU Waste Storage Categories - INEL/P1ywood Boxes (Standard).............. 65

3.22 Content Data for INEL Standard Plywood Boxes............. 66

3.23 Detailed Data on Contact-Handled TRU Waste Storage

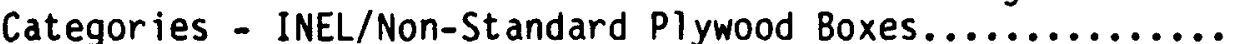

3.24 Detailed Data on Contact-Handled TRU Waste Storage Categories - INEL/Metal Bin....................... 68

3.25 Content of INEL Metal Bins....................... 69

3.26 Detailed Data on Contact-Handled TRU Waste Storage Categories - LANL/30-Gallon Drums...................... 70

3.27 Content of LANL $30-$ Galion Drums.................... 71

3.28 Detailed Data on Contact-Handled TRU Waste Storage Categories - LANL/55-Gallon Drums.................... 72

3.29 Content of LANL $55-$ Gallon Drums...................... 73

3.30 Detalled Data on Contact-Handled TRU Waste Storage Categories - LANL/Corrugated Metal Pipe................. 74

3.31 Detailed Data on Contact-Handled TRU Waste Storage Categories - LANL/83-Gallon Drums.................... 75

3.32 Detalled Data on Contact-Handled TRU Waste Storage Categories - LANL/Non-St andard Drums.................. 76

3.33 Detalled Data on Contact-Handled TRU Waste Storage Categories - LANL/Plywood Boxes...................... 77

3.34 Content of LANL FRP Boxes....................... 78 
3.35 Detailed Data on Contact-Handled TRU Waste Storage Categories - LANL/Concrete Cask........................ 79

3.36 Content of LANL Concrete Casks........................ 80

3.37 Detailed Data on Contact-Handled TRU Waste Storage Categories - NTS/55-Gallon Drums..................... 81

3.38 Detailed Data on Contact-Handled TRU Waste Storage Categories - NTS/Steel Boxes............................ 82

3.39 Detailed Data on Contact-Handled TRU Waste Storage Categories - ORNL/30-Galion Drums.................... 83

3.40 Detailed Data on Contact-Handled TRU Waste Storage Categories - ORNL/55-Gallon Drums..................... 84

3.41 Detailed Data on Contact-Handled TRU Waste Storage Categories - ORNL/Non-Standard Drums

3.42 Detailed Data on Contact-Handled TRU Waste Storage

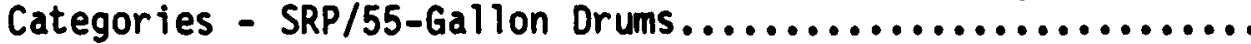

3.43 Detailed Data on Contact-Handled TRU Waste Storage

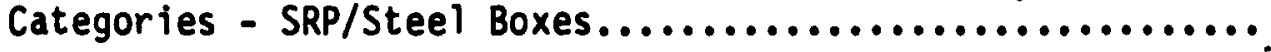

3.44 Detailed Data on Contact-Handled TRU Waste Storage

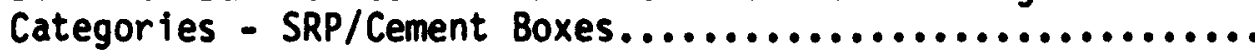

3.45 Detailed Data on Contact-Handled TRU Waste Storage. Categories - SRP/Non-St andard Concrete Boxes..............

3.46 Detailed Data on Contact-Handled TRU Waste Storage Categories - SRP/Concrete Culverts.....................

3.47 Reported Inventories of Remote-Handled Transuranic Waste to Dec. 31, 1980.

3.48 Major TRU Radionuclide Content in Remote-Handled

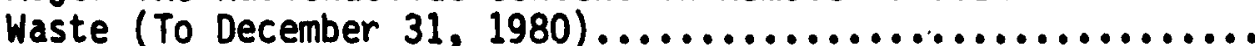

3.49 Remote-Handled Waste Categories at TRU Waste Storage Sites (To December 31, 1980)...

3.50 Detalled Data on Remote TRU Waste Storage Categories - Hanford/Paint 
3.51 Detailed Data on Remote TRU Waste Storage Categories - Hanford/Railroad Flat Cars................ 97

3.52 Detailed Data on Remote TRU Waste Storage Categories - Hanford/Non-Standard Casks................. 98

3.53 Detailed Data on Remote TRU Waste Storage Categories - INEL/30-Gallon Drums (Remote-Handled)......... 99

3.54 Detailed Data on Remote TRU Waste Storage Categories - INEL/Stee 1 Inserts...................... 100

3.55 Detailed Data on Remote TRU Waste Storage Categories - LANL/Concrete Cylinders.................. 101

3.56 Detailed Data on Remote TRU Waste Storage Categories - ORNL/Concrete Casks..................... 102

3.57 Detailed Data on Remote TRU Waste Storage Categories - ORNL/Stainless Steel Capsules............... 103

4.1 Estimated Amounts of Buried TRU Waste.................. 106

4.2 Buried Waste Categories at DOE Storage Sites............. 107

4.3 Summary of Containers of Solid Wastes Shipped From Rocky Flats Plant to the INEL Storage Site, 1954 to 1970)... 108

4.4 Significant Dates and Events Affecting Buried Wastes....... 109

5.1 Description of Site Automated Data Systems.............. 112 


\section{FIGURES}

$\underline{\text { Page }}$

1.1 Locations of DOE Facilities That Generate and/or

Store Defense TRU Wastes.......................... 2

1.3 Buried and Stored TRU Waste at Department of Energy

Sites (To December $31 ; 1980$ )....................... 4

2.2 Forecast of TRU Generation Rate at Department of Energy Sites From 1982-1990 (Contact Handled)............ g

2.3 Forecast of Contact Handled Waste Forms and Containers...... 10

2.19 Remote-Handled Transuranic Waste Projections

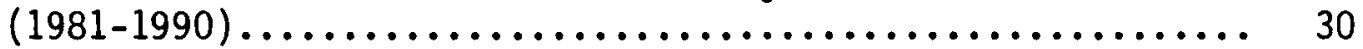

2.20 Forecast of Remote-Handled Waste Forms and Waste

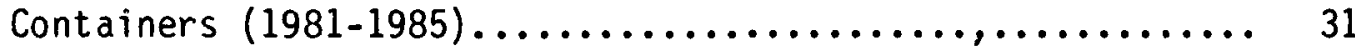

3.2 Contact Handled Waste Forms and Waste Containers

in Storage to December $31,1980 . \ldots \ldots \ldots \ldots \ldots \ldots \ldots \ldots \ldots \ldots .40$

3.3 Current and Projeted Inventories of

Stored TRU Waste at DOE Sites.

3.7 Histogram of $\mathrm{Pu}$ Content in Drums Stored at Idaho

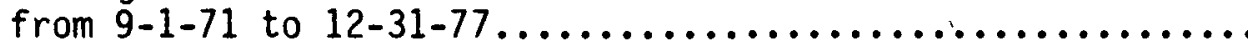

3.8 Histogram of $\mathrm{Pu}$ Content in Boxes Stored at Idaho

from $9-1-71$ to $12-31-77 . \ldots \ldots \ldots \ldots \ldots \ldots \ldots \ldots \ldots \ldots \ldots . . . \ldots \ldots$

3.9 Histogram of Weapon and Heat Source Plutonium Content

in 55-Gallon Drums Produced by Savannah River from

$7-1-74$ to $6-31-77$.

For ease in reading this report, the numbering scheme for tables and figures has been combined. 


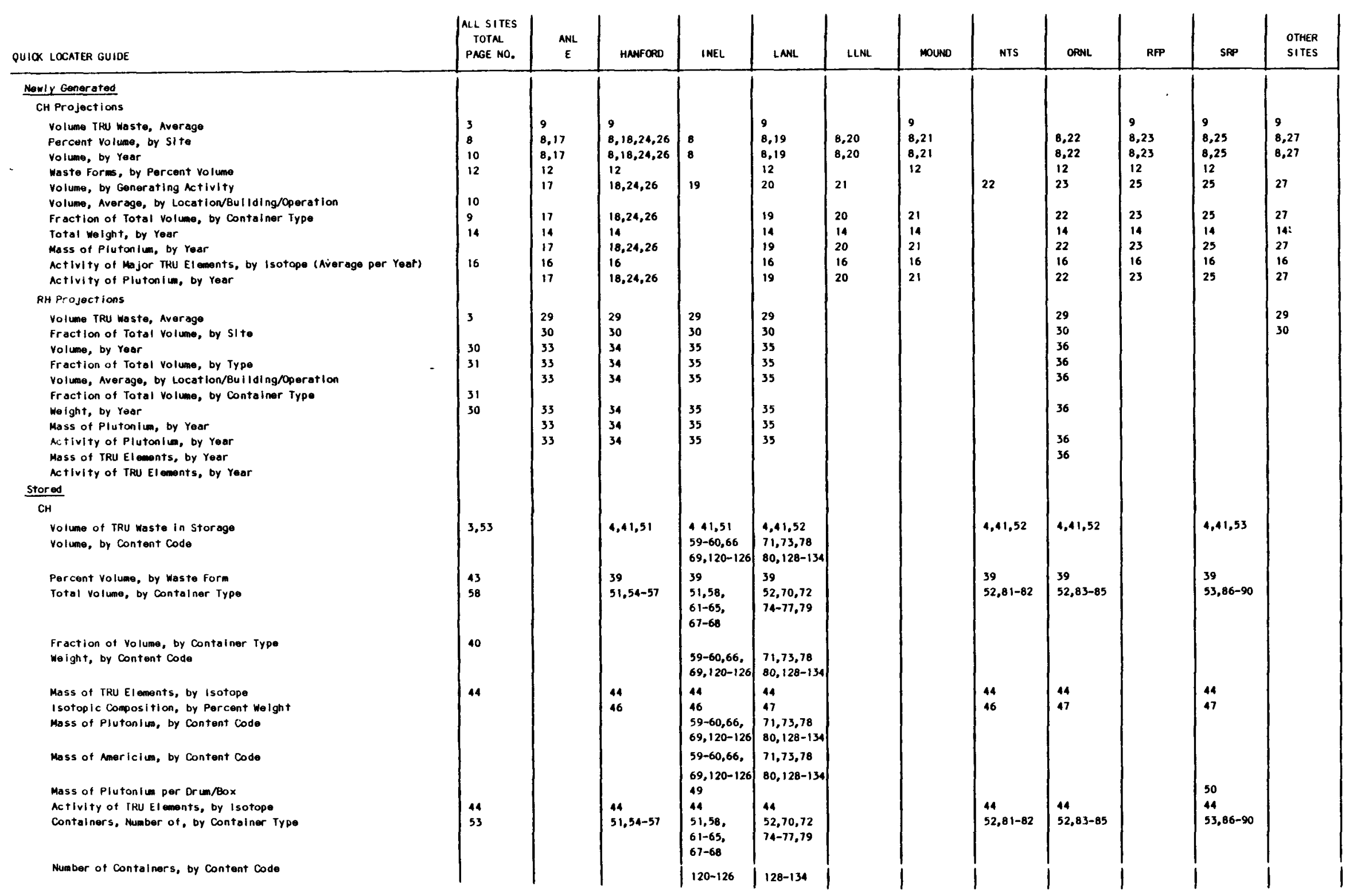




\begin{tabular}{|c|c|c|c|c|c|c|c|c|c|c|c|c|}
\hline QUIOK LOCATFR GUIDE & $\begin{array}{c}\text { ALL SITES } \\
\text { TOIAL } \\
\text { PAGE NO. }\end{array}$ & $\stackrel{A N L}{E}$ & HANFORD & INEL & LANL & LLNL & MOUNE & Nis & ORNL ${ }^{\circ}$ & RFP & sep & $\begin{array}{l}\text { OTHER } \\
\text { SITES }\end{array}$ \\
\hline \multicolumn{13}{|l|}{ Stored } \\
\hline \multicolumn{13}{|l|}{$\mathrm{CH}$} \\
\hline Number of Containers, by Content Code & & & & $120-126$ & $128-134$ & & & & & & & \\
\hline Volume of One Container, by Container Type & & & 51 & 51 & & & & 52 & 52 & & 53 & \\
\hline Activlty per Container (Averago), by Container Type & & & 51 & & & & & 52 & 52 & & 53 & \\
\hline Activity per Container (Range), by Contalner Type & & & 51 & 51 & 52 & & & 52 & 52 & & 53 & \\
\hline $\begin{array}{l}\text { Contalner Dose (Renge), by Container Code } \\
\text { Content Descriptlon, by Contoiner Type }\end{array}$ & & & $54-57$ & $\begin{array}{l}58,61-65 \\
67-68\end{array}$ & $\begin{array}{l}128-134 \\
70,72 \\
74-77,79\end{array}$ & & & $81-82$ & 83-85 & & $86-90$ & \\
\hline Contalner Description, by Container Type & & & $54-57$ & $58,61-65$ & 70,72, & & & $81-82$ & $83-85$ & & $86-90$ & \\
\hline & & & & $67-68$ & $74-77,79$ & & & & & & & \\
\hline Contalner Placement in Storage, by Contalner Type & & & $54-57$ & $\begin{array}{l}58,61-65 \\
67-68\end{array}$ & $\begin{array}{l}70,72 \\
74-77,79\end{array}$ & & & $81-82$ & $83-85$ & & 86-90 & \\
\hline Content Coda Description, by Content Code & & & & $59-6066$, & $71,73,78$ & & & & & & & \\
\hline & & & & 69 & 80 & & & & & & & \\
\hline Radiation Level, by Container Typo & & & $54-57$ & $58,61-65$ & 70,72 & & & $81-82$ & $83-85$ & & $86-90$ & \\
\hline \multirow{2}{*}{\multicolumn{13}{|c|}{ 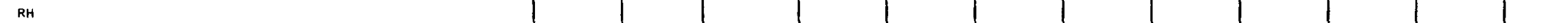 }} \\
\hline volume TRU Waste in Storage & & & $4,92,94$ & & & & & & & & & \\
\hline $\begin{array}{l}\text { Volume TRU Waste in Storage } \\
\text { volume by Contalner Type }\end{array}$ & 13,92 & & $94,96-98$ & $94,99-100$ & $\begin{array}{l}4,92,94 \\
94,101\end{array}$ & & & & $\begin{array}{l}4,92,95 \\
95,\end{array}$ & & & \\
\hline & & & & & & & & & $102-103$ & & & \\
\hline Mass of TRU El emants & 92 & & 92 & 92 & 92 & & & & 92 & & & \\
\hline Mass of TRU Elements, by Isotope & & & 93 & 93 & 93 & & & & 93 & & & \\
\hline isotop ic Composition, by Percent Weight & & & 46 & 46 & 47 & & & & 47 & & & \\
\hline Activity of TRU El ements, by Isotope & & & 93 & 93 & 93 & & & & 93 & & & \\
\hline Volume of One container, by Contalner Type & & & & & & & & & & & & \\
\hline Containers, Number of, by Contalner Type & & & $94,96-98$ & $94,99-100$ & 94,101 & & & & 95, & & & \\
\hline & & & & & & & & & $102-103$ & & & \\
\hline TRU Activity per Contoiner (Average), by Contalner Type & & & 94 & 94 & 94 & & & & 95 & & & \\
\hline Tru Activity per Container (Range), by Container Type & & & 94 & & 94 & & & & & & & \\
\hline Content Description, by Container Type & & & $96-98$ & $99-100$ & 101 & & & & $102-103$ & & & \\
\hline Container Description, by Container Type & & & 96-98 & $99-100$ & 101 & & & & $102-103$ & & & \\
\hline Contalner Placement in Storage, by Contalner Type & & & $96-98$ & $99-100$ & 101 & & & & $102-103$ & & & \\
\hline Radiation Level, by Container Type & & & $96-98$ & $99-100$ & 101 & & & & $102-103$ & & & \\
\hline \multicolumn{13}{|l|}{ Buried } \\
\hline volume of Solid waste Buried & & & & & & & & & & & 4,106 & 106 \\
\hline Vuiume of Contaminated Soll from Solld Waste Burlal & 3,106 & & 106 & 106 & 106 & & & 106 & 106 & & & 106 \\
\hline volume of Conteminated soll from Liquild Waste disposal or Spills & & & 106 & & 106 & & & 106 & 106 & & & \\
\hline Yolume, by Container Type & 107 & & 107 & 107 & 107 & & & 107 & 107 & & 107 & \\
\hline Mass of TRU Elements & 106 & & 106 & 106 & 106 & & & 106 & 106 & & 106 & 106 \\
\hline Mass of TRU Elements from Liquid Waste Di sposal & & & & & 106 & & & & 106 & & & \\
\hline Isotoplc Composition, Percent woight & & & & 46 & 47 & & & & 47 & & & \\
\hline Mass of TRU Elements trom Solld waste Burlal & & & 106 & 106 & 106 & & & 106 & 106 & & 106 & 106 \\
\hline Activity from Solld Waste Burlal & 106 & & 106 & 106 & 106 & & & 106 & 106 & & 106 & \\
\hline Activity of TRU El ements, by Container Type & 107 & & 107 & 107 & 107 & & & 107 & 107 & & 107 & \\
\hline Containers, Number of, by rear and by Container Type & & & & & & & & & & 108 & & \\
\hline
\end{tabular}




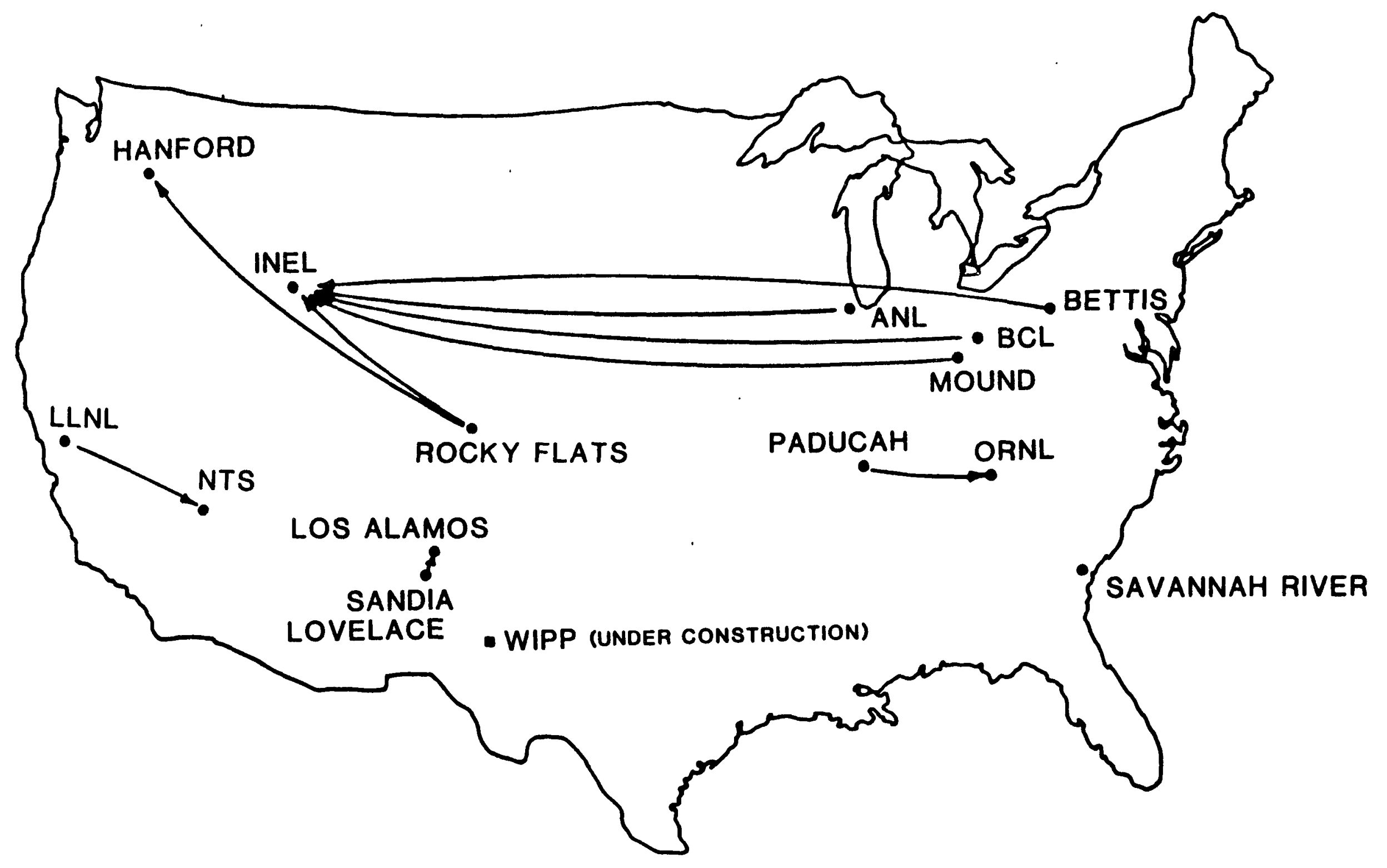

Figure 1.1. Locations of DOE Facilities That fienerate and/or Store Defense TRU Wastes 
Table 1.2. Major Classifications of TRU Waste
Category
Amount $\left(\mathrm{m}^{3}\right)$
Comment

Newly-Generated TRU Waste

Contact-Handled

Remote-Handled

TOTAL
Average Generation per year (1981-90) 4858 87 4945
Newly generated refers to TRU waste that is in process, or that is currently being generated. Contact handled generally refers to waste that is less than $200 \mathrm{mR} / \mathrm{hr}$ at the surface. Remote handled refers to TRU waste that must be handled remotely due to the levels of penetrating radiation given off.
Total in Storage to

Dec. 31,1980

Stored TRU Waste

$\omega$

$\begin{array}{lr}\text { Contact-Handled } & 60,064 \\ \text { Remote-Handled } & 1,819 \\ \text { TOTAL } & 61,883\end{array}$

Total Buried to Dec. 31,1980

Buried TRU Waste

\section{Buried Waste}

298,933

Contaminated Soil

TOTAL
131,500 to $2,595,000$

430,433 to $2,893,933$
Stored waste refers to TRU waste that has been segregated from other radioactive waste when it was suspected or known to contain significant quantities of TRU radionuclides.
Buried waste is TRU waste that has been buried in pits and trenches. It can also refer to underground material that has become contaminated with TRU radionuclides. May not be segregated from wastes which are less than $10 \mathrm{nCi} / \mathrm{g}$. 


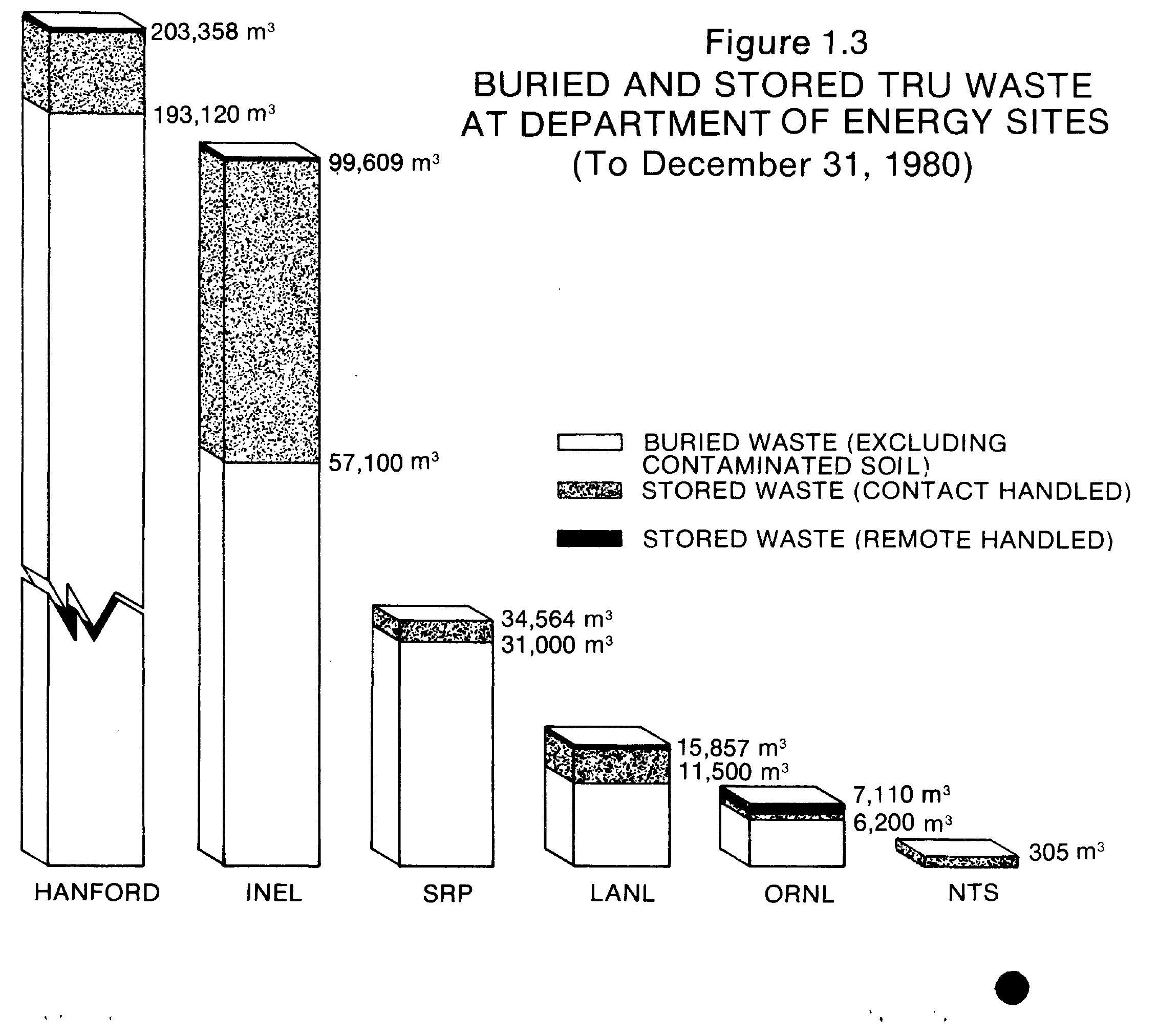


the waste. These tables include the three most common TRU isotopes and present typical compositions of the two common types of plutonium.

After the summary tables, a series of charts detail similar information for ten individual sites. The contact-handled waste is covered first, followed by a summary and details on remote-handled waste. Five sites are included on remote-handled waste. These tables show where TRU waste is generated at each site, and the activity that generates it.

Section 3 contains data on Stored Waste. As in Section 2, summary tables are given first. The tables show the locations, quantities, and waste forms for six storage sites. Figures are included which indicate total percentages of waste forms and waste containers. A discussion of the contribution of the actual contents versus the volume of the containers is included. Total isotopes at the six sites, as well as the estimated isotopes in particular types of waste, are given.

A detailed review of each site follows the summary tables. The review is separated by contact- and remote-handled waste. Each part of this review begins with a summary table showing the number and kinds of containers used to store the waste. Following the summary table, each type of container is detailed for all six sites. A description of the container is followed by data on the placement, inside configuration, and all available data on the contents of each container. The TRIJ activity present and the radiation level are given for some. Data on the radionuclides and radiation level are often available in more detail from the site.

Section 4 presents all of the available data on Buried Wastes. Very few records have been kept on Buried Waste. The data consist primarily of estimates of the total waste and the resulting contaminated soil. A limited amount of data were obtained on the types of containers and contents. 
ORNL and SRP both have a record of the number of concrete monoliths that were buried. These are presented along with the total number of cubic meters of boxes, drums, and other miscellaneous containers at the other sites. A table showing the amount and types of containers that Rocky Flats shipped to Idaho from 1954 to 1970 is also presented. This gives an indication of the types of burial that were practiced throughout all AEC sites. Finally, a chronological table of events affecting Buried Waste is included.

The final section, Section 5, was included to provide readers with a sample of other kinds of data that are available. Information is given on data at the various sites, as well as other studies which have been conducted.

Throughout this report two kinds of notation were used when data could not be found for a particular table. Each notation implies a separate reason for the lack of data. The symbol N/A means "not available," and implies that a record was not kept and further research would probably not result in more data. The symbol N/R is used to mean "not reported." This implies that the data are possibly available, but due to lack of time to research the data, they were not included. 


\section{CHARACTERISTICS OF NEWLY GENERATED TRU WASTE}

\subsection{Contact-Handled - Summary}

\subsubsection{Locations, Quantities, and Waste Forms}

The DOE sites where contact-handled TRU waste will be generated for the next 10 years are 1 isted in Table 2.1 and shown in Figure 2.2. These data were compiled from the tables found in Section 1.2 of this report. As in any forecast, the volumes listed cannot be completely accurate. Generally, the forecasts are based upon recent trends with allowances for new projects or the phasing out of old ones. In the next 5 years, the RFP, and the LANL, expect to reduce some kinds of waste generation. Increases in other waste streams, however, will result in a slight increase in the overall amount of TRU waste. Increases are also expected at the Hanford site. These increases will result from the Fast Flux Test Facility (FFTF) activites, additional D\&D projects, and the startup of the reprocessing facility on the Hanford site. The Mound Facility generation rate will increase and then decrease as D\&D projects are completed. Figure 2.2 shows the relative contributions of the major sites, and the total volume and weight of the expected generation. The differences are within the expected deviations, and overal1, the rate will be about $4,900 \mathrm{~m}^{3}$ per year. A slight decrease is seen for the years 1986-1990. However, this is probably due to uncertainty in forecasting, rather than a real decrease.

Table 2.1 also shows the expected waste forms. These percentages are based upon the most recent year-of-operation data available. As seen, the data are somewhat incomplete because records are not readily available at some sites. Many records distinguish only between combustible and noncombustible. By going to the production facilities, or old burial records, some data were obtained. These percentages are not expected to remain constant. Many activities are currently underway which will reduce the amount of combustibles. Also, the amount of 
Table 2.1 Projection of Contact Handled TRU Waste - Volumes and Waste Forms

Volume, $\mathrm{m}^{3}$

Volume

\begin{tabular}{|c|c|c|c|c|c|c|c|c|c|c|c|c|c|c|}
\hline Sites & 1981 & 1982 & 1983 & 1984 & 1985 & $\begin{array}{l}\text { Avg/yr } \\
1986-90 \\
\end{array}$ & Combustibles & $\begin{array}{c}\text { Glass } \\
\text { and } \\
\text { Motal }\end{array}$ & $\begin{array}{l}\text { Filter } \\
\text { and } \\
\text { Filter } \\
\text { Media }\end{array}$ & $\begin{array}{l}\text { Absorbed } \\
\text { Li quids, } \\
\text { Dried } \\
\text { Salts } \\
\text { (Sludge) }\end{array}$ & $\begin{array}{c}\text { Dirt } \\
\text { Ground } \\
\text { or } \\
\text { Asphalt } \\
\end{array}$ & $\begin{array}{c}\text { Concrete } \\
\text { and } \\
\text { Cemented } \\
\text { Waste }\end{array}$ & Other & Ref \\
\hline Argonne-East & 270 & 180 & 60 & 60 & 60 & 60 & $42 \%$ & $34 \%$ & a & $3 \%$ & $<1 \%$ & $0 \%$ & $21 \%$ & 4,5 \\
\hline Argonne-West & .6 & .6 & .8 & .8 & .8 & .8 & $50 \%$ & * & * & * & $0 \%$ & $0 \%$ & $50 \%$ & 5 \\
\hline Battel le-Col unbus & 50 & 204 & 0 & 0 & 0 & 0 & $20 \%$ & * & * & * & * & * & $80 \%$ & 5 \\
\hline Battelle-Paclfic NW Lab & 955 & 37 & 28 & 34 & 31 & 34 & $38 \%$ & $35 \%$ & * & $<1 \%$ & $0 \%$ & $0 \%$ & $27 \%$ & 6,8 \\
\hline Bett is & 25.6 & 27 & 11 & 15.1 & 15.1 & 10 & $38 \%$ & * & * & * & * & * & $62 \%$ & 5 \\
\hline Hanford-(Offsite) & 232 & 580 & 297 & 245 & 269 & 269 & * & * & * & * & * & * & * & 7 \\
\hline IOPP & 44 & 2 & 2 & 2 & 2 & 2 & $50 \%$ & $50 \%$ & $0 \%$ & $0 \%$ & $0 \%$ & $0 \%$ & $0 \%$ & 5 \\
\hline INEL Test Reactor Area & .4 & .4 & .4 & .4 & .4 & .4 & * & * & * & * & * & * & * & 5 \\
\hline LANL & 850 & 730 & 540 & 560 & 580 & 598 & $33 \%$ & $25 \%$ & $2 \%$ & $7 \%$ & $3 \%$ & $10 \%$ & $20 \%$ & 9,10 \\
\hline LLNL & 24.8 & 25 & 25 & 25 & 25 & 25 & $27 \%$ & $65 \%$ & * & * & $0 \%$ & $8 \%$ & $0 \%$ & 1 \\
\hline Lovelace & 2 & 2 & 2 & 2 & 2 & 2 & * & * & * & * & * & * & * & 1 \\
\hline Mound Facllity & 530 & 885 & 780 & 780 & 780 & 420 & $7 \%$ & $65 \%$ & $<1 \%$ & $8 \%$ & $0 \%$ & $20 \%$ & $<1 \%$ & 11 \\
\hline ORNL & 29.4 & 30 & 30 & 30 & 30 & 30 & $60 \%$ & $15 \%$ & $b$ & b & $0 \%$ & $0 \%$ & $25 \%$ & 12 \\
\hline RHO & 808 & 220 & 376 & 410 & 651 & 560 & $59 \%$ & $27 \%$ & * & $<1 \%$ & * & * & $14 \%$ & 6,8 \\
\hline$R \mid$ I RFP & 1650 & 1650 & 1790 & 2050 & 1890 & 1820 & $39 \%$ & $25 \%$ & $11 \%$ & $9 \%$ & $3 \%$ & $7 \%$ & $6 \%$ & 3 \\
\hline Sandia & 1 & 1 & 1 & 1 & 1 & 1 & & * & * & * & * & * & * & 1 \\
\hline SRP & 370 & 445 & 468 & 515 & 552 & 580 & $82 \%$ & $17 \%$ & a & $0 \%$ & $0 \%$ & $0 \%$ & $1 \%$ & 13,14 \\
\hline Un ited Nuclear Company & 0 & 6 & 6 & 6 & 6 & 6 & $0 \%$ & $0 \%$ & $100 \%$ & $0 \%$ & $0 \%$ & $0 \%$ & $0 \%$ & 6,8 \\
\hline Westinghouse Hanford Co. & 38 & 506 & 331 & 76 & 142 & 141 & 438 & $23 \%$ & a & $18 \%$ & $0 \%$ & $0 \%$ & $13 \%$ & 6,8 \\
\hline
\end{tabular}

Total

$\begin{array}{llllll}5881 & 5531 & 4748 & 4812 & 5037 & 4559\end{array}$

* Not available

a Included in combustibles.

b Included in "other" category. 
Figure 2.2

FORECAST OF TRU GENERATION RATE AT

DEPARTMENT OF ENERGY SITES FROM 1982-1990

(Contact Handled)
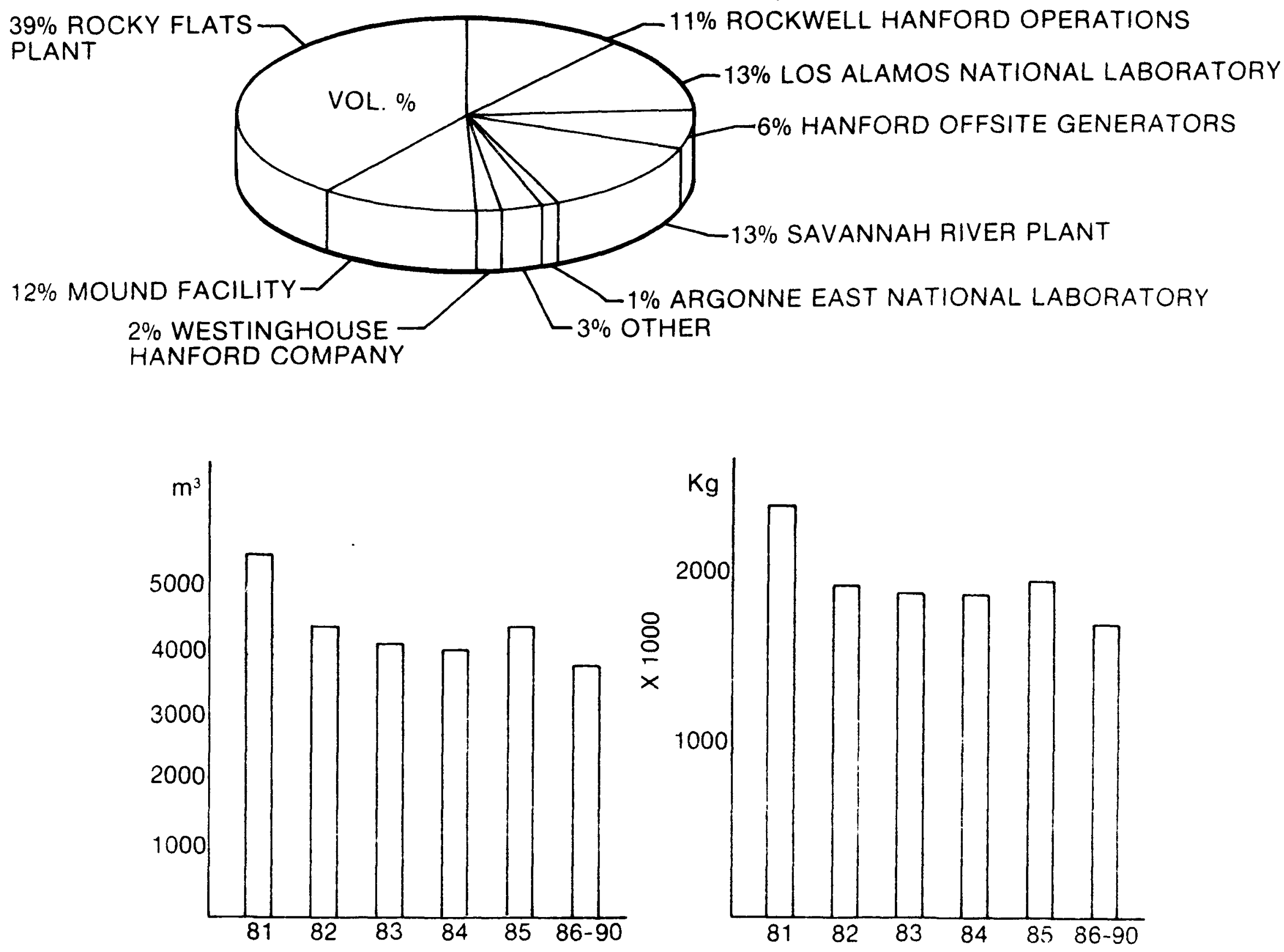


\section{Figure 2.3 \\ FORECAST OF CONTACT HANDLED WASTE FORMS AND CONTAINERS (1981-1985)}

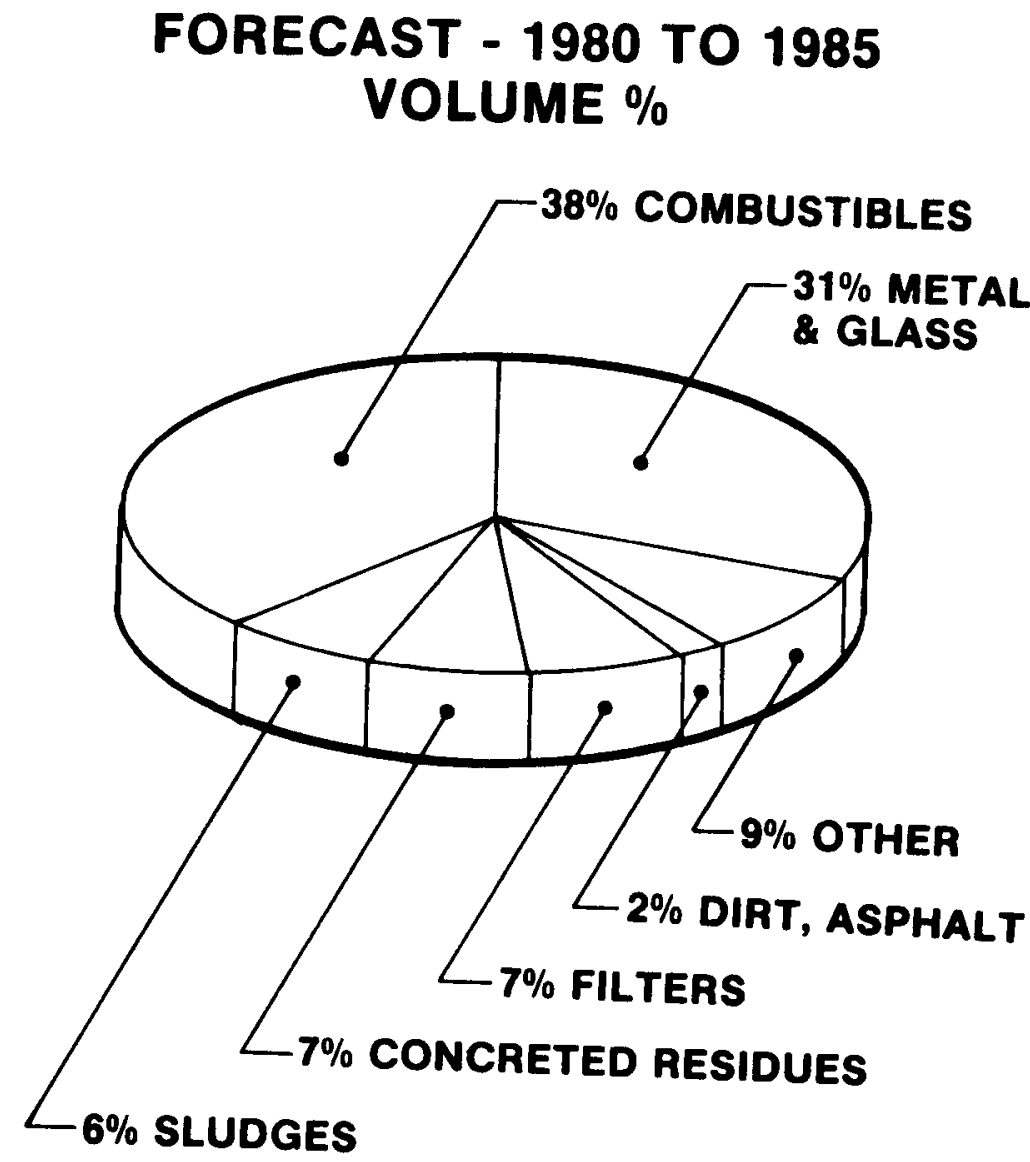

FORECAST - 1980 TO 1990

VOLUME \%

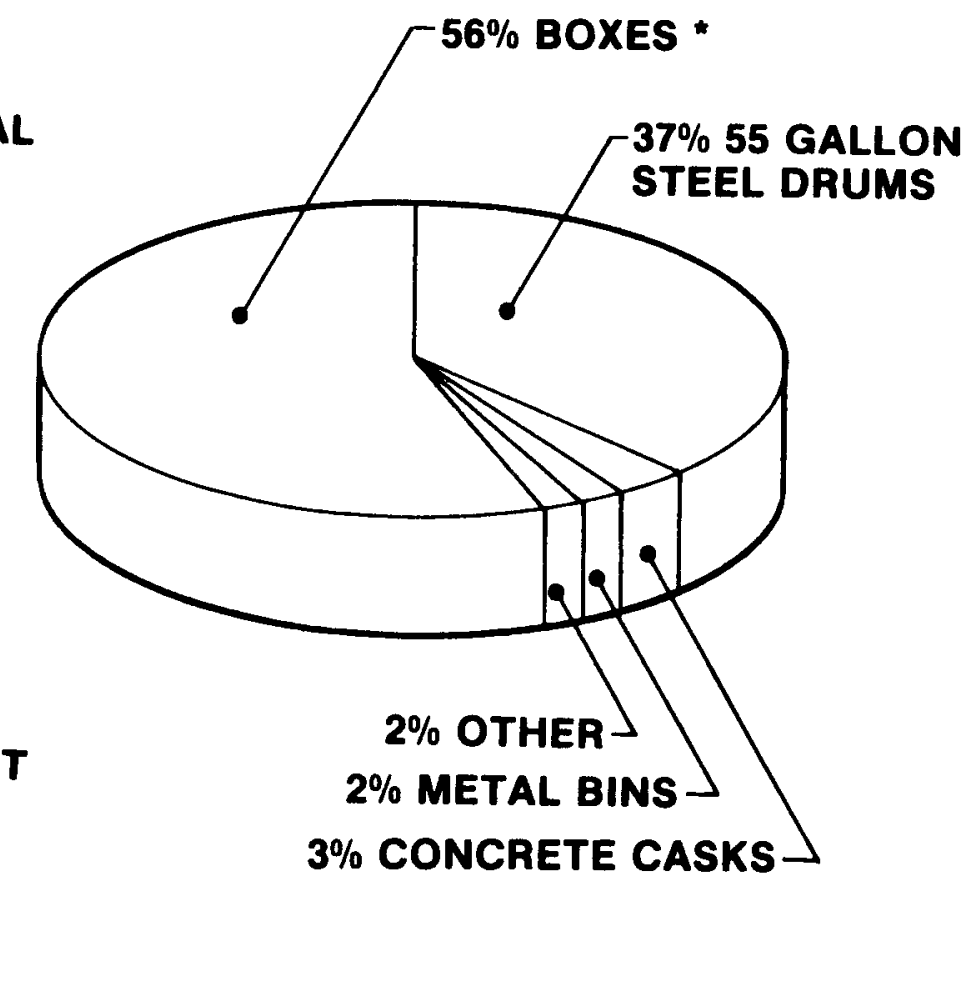

* PLYWOOD UNTIL 1983 - METAL THEREAFTER 
concrete or cemented waste is expected to increase. This will result from process changes being used to stabilize process wastes for storage in the WIPP. More D\&D projects will add to the percentage of metal. Figure 2.3 shows the estimated percentage of each waste form for the total DOE complex. It also shows the present percentages of containers used to store the waste. The type of containers had changed somewhat during the past 10 years, but have become standard in recent years. Some additional changes will now be made for shipping to the Waste Isolation Pilot Plant (WIPP).

\subsubsection{Activities That Generate TRU Waste}

Table 2.4 shows the activities that generate TRU waste. TRU waste comes from three major processes. The largest source is production lines at RFP, the LANL, and RHO. Nearly one-half of all TRU waste comes from weapons components manufacture at Rocky Flats and subsequent plutonium recovery at the three sites. This does not include D\&D waste that results from those activities. ${ }^{3}$ The second largest source of TRU waste comes from D\&D projects. They account for one-fourth of all TRU waste. As the Mound Facility phases out their recovery and encapsulating work, they will generate 40 percent of all D\&D wastes. At most sites the amount of D\&D wastes will continue to increase. As the percentage of D\&D waste increases, and reductions are made in the amount of production waste, the D\&D waste will become the largest source of TRU waste. Finally, about 20 percent of TRU wastes will come from laboratory activities. ${ }^{3}$ These wastes are the most difficult to characterize. Nearly all of the exotic TRU isotopes arise from these activities. The category also includes all wastes that must be shielded due to penetrating radiation [greater than $200 \mathrm{millirem}$ per hour $(\mathrm{mr} / \mathrm{hr}$ ) at the surface of the container].

\subsubsection{TRU Content}

TRU content is determined by the process which generates the waste. Generally, waste from a given process will not be mixed with 
Table 2.4 Activities That Generate TRU Waste ${ }^{3}$

(Cubic Meters Per Year 1981-1985)

\begin{tabular}{|c|c|c|c|c|c|c|c|c|}
\hline \multirow[b]{2}{*}{ Major Sites } & \multicolumn{4}{|c|}{ Production } & \multicolumn{2}{|c|}{ Laboratory } & \multirow[t]{2}{*}{$D \& D$} & \multirow[b]{2}{*}{ Total } \\
\hline & $\begin{array}{r}\text { Foundry \& } \\
\text { Fabrication } \\
\end{array}$ & $\begin{array}{c}239 \mathrm{Pu} \\
\text { Recovery } \\
\end{array}$ & $\begin{array}{c}\mathrm{Pu} \\
\text { Reprocessing } \\
\end{array}$ & $\begin{array}{l}\text { Heat } \\
\text { Source } \\
\text { Work } \\
\end{array}$ & $\begin{array}{c}\text { Analytical } \\
\text { Labs } \\
\end{array}$ & $\begin{array}{l}\text { R\&D } \\
\text { Labs }\end{array}$ & & \\
\hline Argonne - East & 0 & 0 & 0 & 0 & $<1$ & 70 & 20 & 90 \\
\hline Hanford & 0 & $310^{*}$ & $30^{*}$ & 0 & $N / A$ & $150^{\star}$ & 540 & 1030 \\
\hline LANL & 160 & 320 & 0 & 40 & $N / A$ & $80^{\star}$ & 60 & 660 \\
\hline Mound & 0 & 0 & 0 & 0 & 170 & 70 & 520 & 760 \\
\hline Rocky Flats & 580 & 870 & 0 & 0 & 130 & 120 & 140 & 1840 \\
\hline ORNL & 0 & 0 & 0 & 0 & $N / A$ & $60^{\star}$ & 0 & 60 \\
\hline Savannah River & 0 & 0 & 130 & 80 & 50 & 40 & 60 & 360 \\
\hline TOTAL & 740 & 1500 & 160 & 120 & 350 & 590 & 1340 & 4800 \\
\hline
\end{tabular}

*Includes Analytical Lab Waste.

N/A - Not Available. 
waste from another process. At the present time, two major processes account for most of the TRU content in the wastes.

First, the fabrication of nuclear weapons generates waste which has large amounts of ${ }^{239} \mathrm{Pu}$. Second, the generation of heat source plutonium results in waste containing ${ }^{238} \mathrm{Pu}$. Other processes generate TRU isotopes such as ${ }^{244} \mathrm{Cm},{ }^{252} \mathrm{Cf}$, and ${ }^{233} \mathrm{U}$, but they are small when compared to the quantities of ${ }^{239} \mathrm{Pu}$ and ${ }^{238} \mathrm{Pu}$. A significant amount of ${ }^{241} \mathrm{Am}$ also results from the recovery and purification of ${ }^{238} \mathrm{pu}$ in the weapons program at RFP. It should be noted that these statements pertain to the weight percent of the nuclides. If the activity (in curies) is measured, ${ }^{241} \mathrm{Pu}$ becomes the largest fraction in the waste stream. This is shown when the isotopic compositions are presented.

Table 2.5 shows a forecast of the three major TRU isotopes found in the wastes. Table 2.6 shows the typical composition of the weapons grade and heat source plutonium. ${ }^{15}$ Small differences may exist at individual sites. The forecast in Table 2.5 was tabulated from the data on individual sites in Section 2.2. It does not include isotopes from remote-handled waste. Slight variations can occur in comparing similar data, depending on the specific activity and isotopic compositions and in calculating the total activity present.

Further data are presented on the isotopic composition at individual storage sites in a later section. Storage data also include specific quantities of many of the TRU nuclides found in the waste. Because many of these isotopes are generated sporadically, however, it was not feasible to make projections on each isotope. 
Table 2.5 Forecast of Major TRU Isotopes Contained in Newly Generated Waste (Average Per Year)

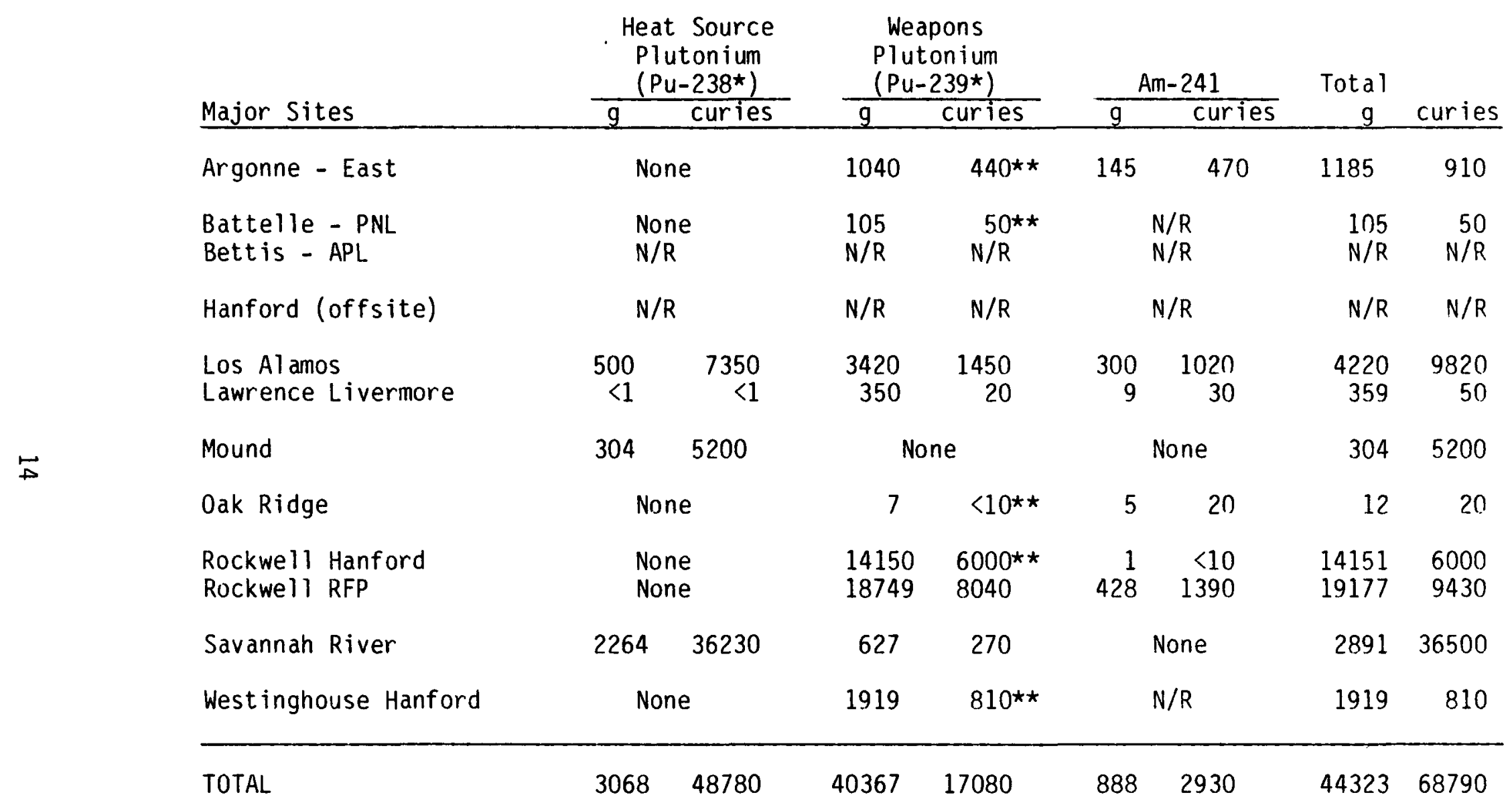

All activities reported here are rounded.

* Includes other plutonium isotopes (see Table 2.6).

$\star \star$ Calculated, assuming weapons grade Pu-239, specific activity $=0.424 \mathrm{Ci} / \mathrm{g} .{ }^{2}$ Gram amounts are as reported by the site.

N/A Not Available

N/R Not Reported 


\section{ERRATA}

Page 14 In Table 2.5 Los Alamos Weapons Plutonium ( $\mathrm{Pu}-239 *$ ) should read 34,200 grams and 14,500 curies. Consequently, the site total should be changed to 35,000 grams and 22,870 curies. All sites total should read 75,103 grams and 81,840 curies. 
TABLE 2.6

Typical Isotopic Composition of Weapon Plutonium

\begin{tabular}{l} 
Isotope \\
\hline $238 \mathrm{Pu}$ \\
$239 \mathrm{Pu}$ \\
$240 \mathrm{Pu}$ \\
$241 \mathrm{Pu}$ \\
$242 \mathrm{Pu}$ \\
$241 \mathrm{Am}$
\end{tabular}

Weight Fraction

$\underline{\mathrm{Ci} / \mathrm{g} \mathrm{Pu}}$

0.0003

0.005

0.939

0.057

0.058

0.003

0.014

0.0003

0.347

0.0002

0.0006

Total 0.424

Typical Isotopic Composition of Heat Source Plutonium

I sotope

$$
\begin{aligned}
& 238 \mathrm{Pu} \\
& 239 \mathrm{Pu} \\
& 240 \mathrm{Pu} \\
& 241 \mathrm{Pu} \\
& 242 \mathrm{Pu}
\end{aligned}
$$

Weight Fraction

$$
\begin{aligned}
& 0.802 \\
& 0.159 \\
& 0.0302 \\
& 0.0064 \\
& 0.0013
\end{aligned}
$$

$\underline{\mathrm{Ci} / \mathrm{gPu}}$

13.95

0.01

0.007

0.72

0

Total 14.7 


\subsection{Review of the Major Sites that Generate Contact-Handled Waste}

Tables 2.7 through 2.16 present the exact locations and sources of TRU waste generation. Sites that currently generate $25 \mathrm{~m}^{3}$ or more per year are included. A summary table (Table 2.17) shows sites that generate less than $25 \mathrm{~m}^{3}$. Each table shows the forecast volume, weight, and plutonium content of the waste. Also included are the facilities that generate the waste, and what percentage each operation contributes to the site total. Finally, a pie chart in the right-hand corner shows the expected volume percent of the various waste forms. These data are expected to remain consistent for at least the next 5 years. 
Table 2.7 Forecast of Transuranic Waste Generation at Argonne National Laboratory - East ${ }^{4}, 5$ (Contact-Handled)

\begin{tabular}{|c|c|c|c|c|c|c|}
\hline & 1981 & 1982 & 1983 & 1984 & 1985 & 1986-90 (Aver age) \\
\hline Volume, $\mathrm{m}^{3}$ & 270 & 180 & 60 & 60 & 60 & 60 \\
\hline Weight*, $\mathrm{kg} \times 10^{3}$ & 81 & 54 & 18 & 18 & 18 & 18 \\
\hline \multicolumn{7}{|l|}{ Pu content } \\
\hline$g$ & 1,040 & 500 & 500 & 500 & 500 & 500 \\
\hline curies** & 441 & 212 & 212 & 212 & 212 & 212 \\
\hline
\end{tabular}

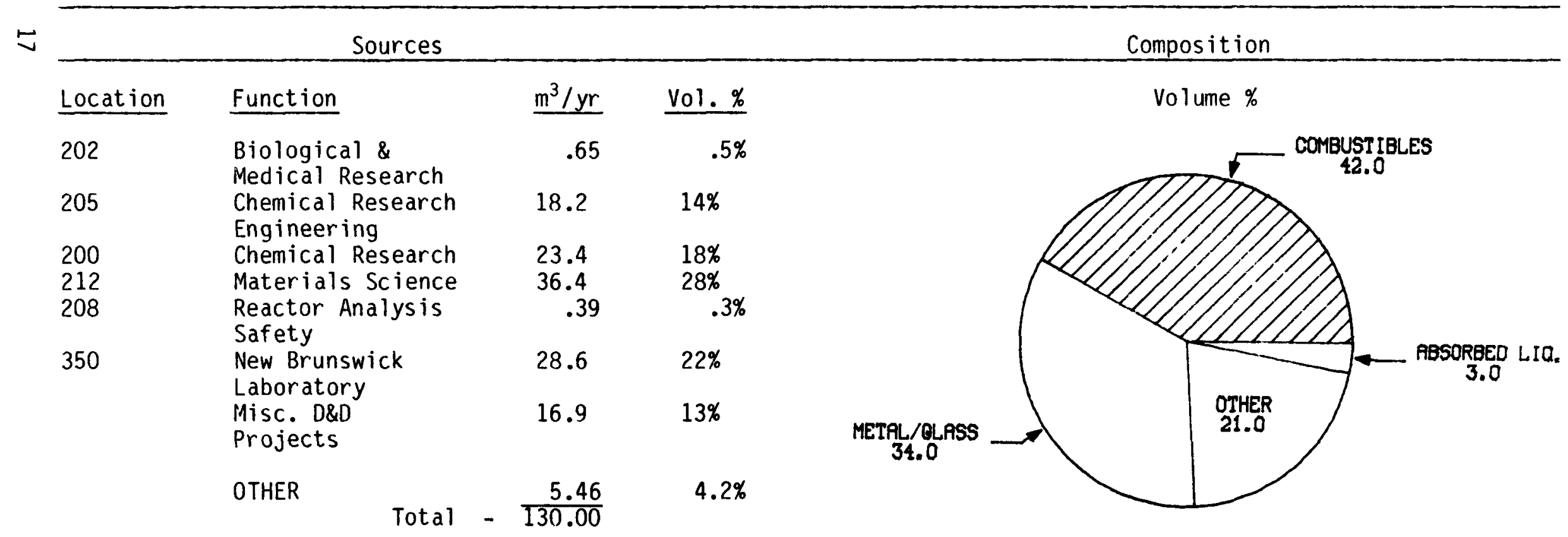


Table 2.8 Forecast of Transuranic Waste Generation at Battelle - PNL ${ }^{6} 8$

(Contact-Handled)

\begin{tabular}{lcccccc} 
& 1981 & 1982 & 1983 & 1984 & 1985 & $1986-90$ (Aver age) \\
\cline { 2 - 7 } Volume, $\mathrm{m}^{3}$ & $955(25.4)$ & 37 & 28 & 34 & 31 & 34 \\
Weight, $\star \star$ \\
Pu content
\end{tabular}

* This actual volume figure includes waste held in temporary storage; i.e., some of the volume included here had been generated in previous years.

** Estimated density at $300 \mathrm{~kg} / \mathrm{m}^{3}$.

$\star \star \star$ Calculated using .424 Ci/g.

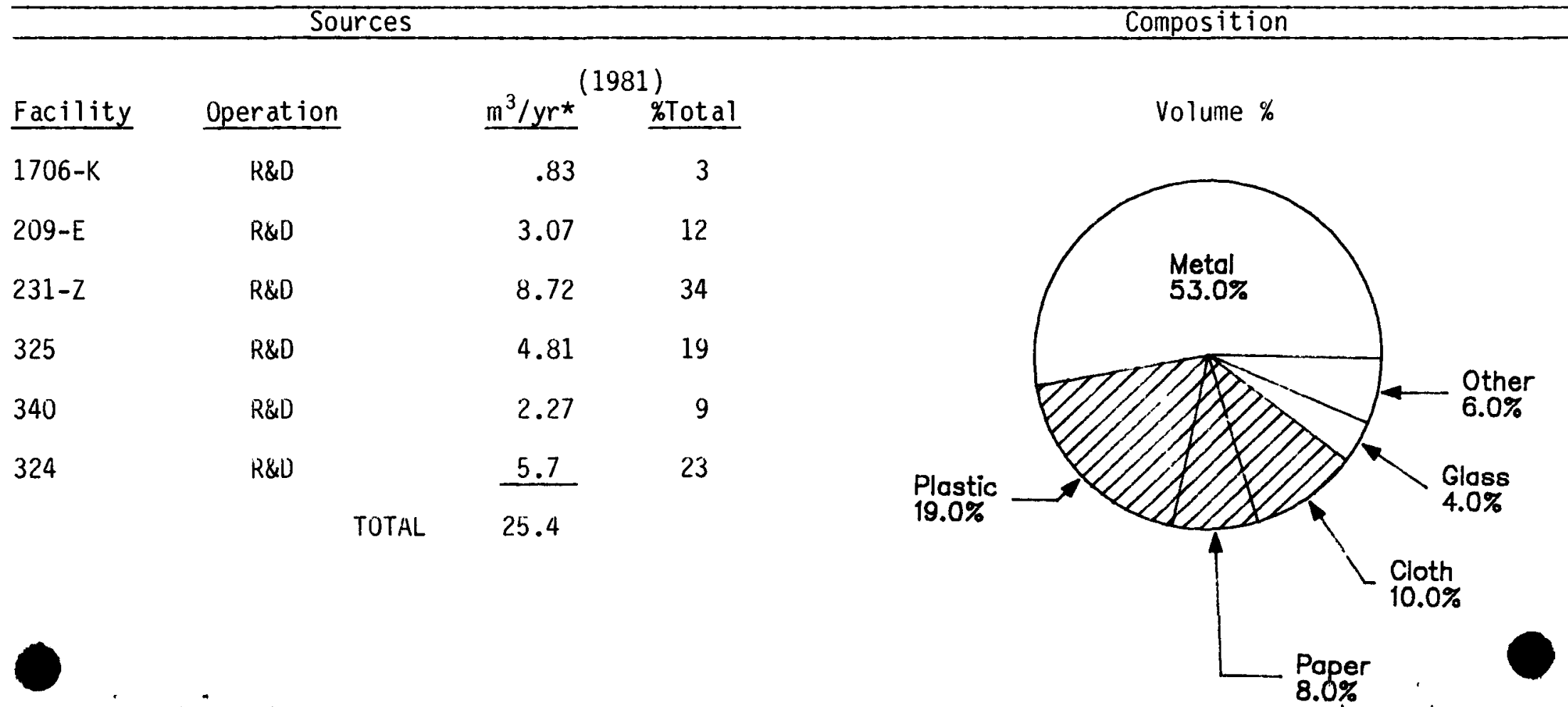


Table 2.9 Forecast of Transuranic Waste Generation at Los Alamos National Laboratory9110 (Contact-Hand led)

\begin{tabular}{|c|c|c|c|c|c|c|}
\hline & 1981 & 1982 & 1983 & 1984 & 1985 & 1986-90 (Average) \\
\hline Volume, $\mathrm{m}^{3}$ & 850 & 730 & 540 & 560 & 580 & 598 \\
\hline Weight, $\mathrm{kg} * \times 10^{3}$ & 382.5 & 328.5 & 243 & 252 & 261 & 269 \\
\hline Pu content & & & & & & \\
\hline$g$ & 34,700 & 34,700 & 34,700 & 34,700 & 34,700 & 34,700 \\
\hline curies & 21,850 & 21,850 & 21,850 & 21,850 & 21,850 & 21,850 \\
\hline
\end{tabular}

* Based on average density calculation for waste generated in $1980\left(450 \mathrm{~kg} / \mathrm{m}^{3}\right)$.

セ

$\begin{array}{ll}\text { Facility } & \text { Operation } \\ \text { TA-55 } & \begin{array}{l}\text { Pu Recovery and Fabricat } \\ \text { TA-3-29 }\end{array} \\ \begin{array}{cc}\text { Chemistry and Metallurgy } \\ \text { Research }\end{array} \\ \text { TA-50 } & \begin{array}{l}\text { Liquid Waste Treatment } \\ \text { Decontamination/ } \\ \text { Decommissioning }\end{array}\end{array}$

Total

\begin{tabular}{|c|c|}
\hline $\begin{array}{c}(1980-1981) \\
\mathrm{m}^{3} / \mathrm{yr}\end{array}$ & \%Total \\
\hline 420 & 70 \\
\hline 50 & 8 \\
\hline 70 & 12 \\
\hline 60 & 10 \\
\hline 600 & 100 \\
\hline
\end{tabular}

Volume $\%$

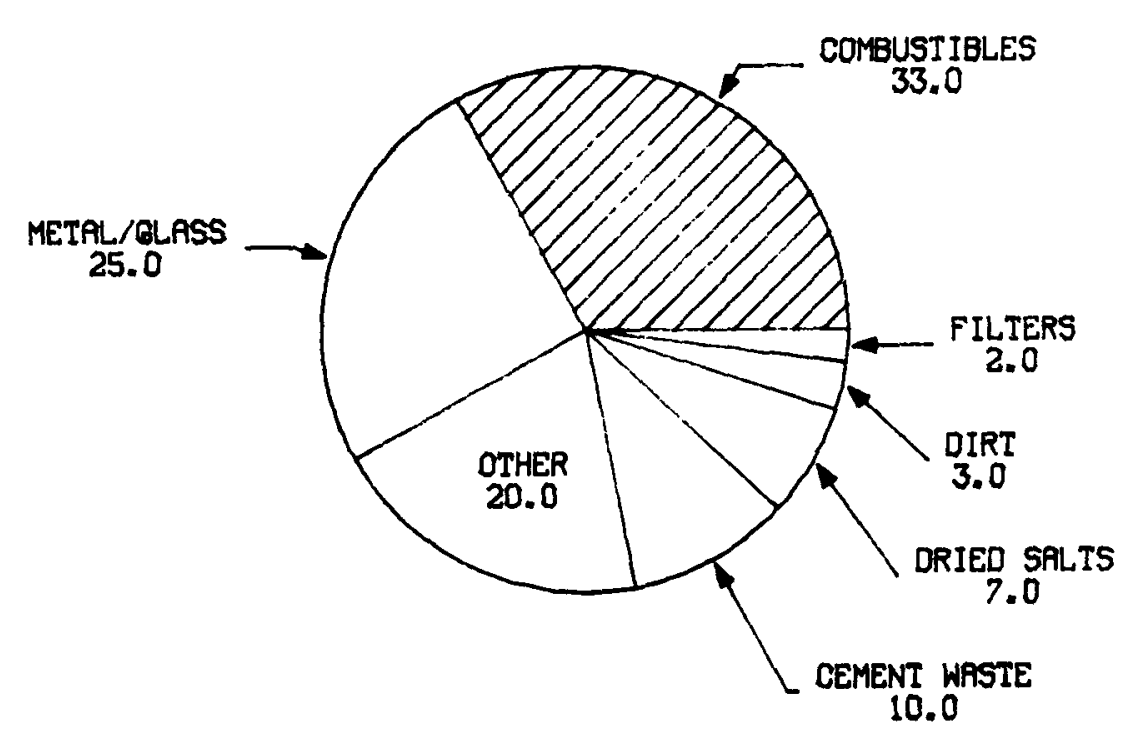


Table 2.10 Forecast of Transuranic Waste Generation at Lawrence Livermore National Laboratory ${ }^{1}$ (Contact-Hand led)

\begin{tabular}{lcccccc} 
& 1981 & 1982 & 1983 & 1984 & 1985 & $1986-90$ (Average) \\
\cline { 2 - 7 } & 24.8 & 25 & 25 & 25 & 25 & 25 \\
Volume, $\mathrm{m}^{3}$ & 7.4 & 7.5 & 7.5 & 7.5 & 7.5 & 7.5 \\
Weight*, $\mathrm{kg} \times 10^{3}$ & & & & & & \\
Pu content & 380 & 380 & 380 & 380 & 380 & 380 \\
$\quad \mathrm{~g}$ & 161 & 161 & 161 & 161 & 161 & 161
\end{tabular}

* Assumes average density at $300 \mathrm{~kg} / \mathrm{m}^{3}$.

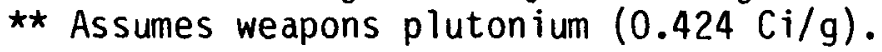

\begin{tabular}{|c|c|c|c|}
\hline Location & Operation & $\begin{array}{c}(1981-1980) \\
\mathrm{m}^{3} / \mathrm{yr}^{\star}\end{array}$ & \%Total \\
\hline $\begin{array}{l}\text { Building } \\
332\end{array}$ & $\begin{array}{l}\text { Plutonium Weapons } \\
\text { Research }\end{array}$ & 25 & $100 \%$ \\
\hline
\end{tabular}

* Approximately 1989-1990 LLNL will begin D\&D project in Building 251. This will double their rate of TRU generation for about 5 years. 
Table 2.11 Forecast of Transuranic Waste Generation at Mound Facility 11

(Contact-Hand led)

\begin{tabular}{|c|c|c|c|c|c|c|}
\hline & 1981 & 1982 & 1983 & 1984 & 1985 & 1986-90 (Average) \\
\hline Volume, $\mathrm{m}^{3}$ & 530 & 885 & 780 & 780 & 780 & 420 \\
\hline Weight*, $\mathrm{kg} \times 10^{3}$ & 419 & 699 & 616 & 616 & 616 & 332 \\
\hline \multicolumn{7}{|l|}{ Pu content** } \\
\hline$g$ & 1,100 & 600 & 500 & 500 & 400 & 200 \\
\hline curies & 16,000 & 9,000 & 8,000 & 8,000 & 6,000 & 3,000 \\
\hline
\end{tabular}

* Based on average density for waste shipped to INEL in $1980\left(790 \mathrm{~kg} / \mathrm{m}^{3}\right)$.

** Assumes all Mound TRU activity is due to Pu-238.

$\simeq$

Sources

Composition

Location Operation

(1981)

Bidg. 38 Decontamination and

Bldg. R Decommissioning at

Inactive all three sites*

Waste

Transfer

System

Analytical Laboratory

117

365

69

R\&D Laboratory

48

22

530

TOTAL

* Includes some production operations which are phasing out.

\%Total
Volume \%

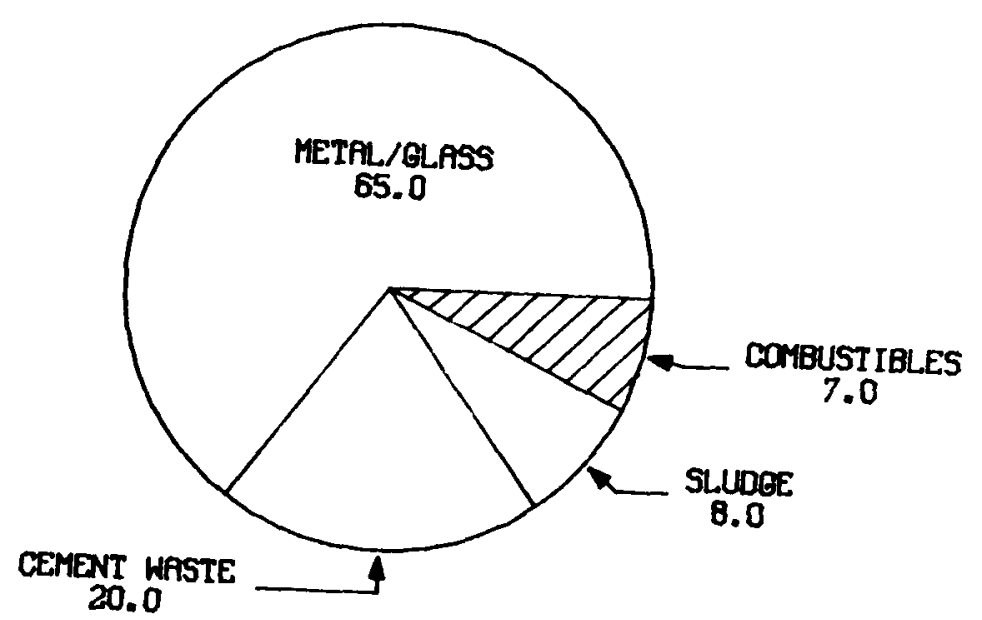


Table 2.12 Forecast of Transuranic Waste Generation at Oak Ridge National Laboratory ${ }^{12}$ (Contact-Handled)

\begin{tabular}{|c|c|c|c|c|c|c|}
\hline & 1981 & 1982 & 1983 & 1984 & 1985 & 1986-90 (Aver age) \\
\hline Volume, $\mathrm{m}^{3}$ & 30 & 30 & 30 & 30 & 30 & 30 \\
\hline Weight $*, \mathrm{~kg} \times 10^{3}$ & 9 & 9 & 9 & 9 & 9 & 9 \\
\hline \multicolumn{7}{|l|}{ Pu content } \\
\hline$g$ & 72 & 72 & 72 & 72 & 72 & 72 \\
\hline curies & 30 & 30 & 30 & 30 & 30 & 30 \\
\hline
\end{tabular}

Sources

Composition

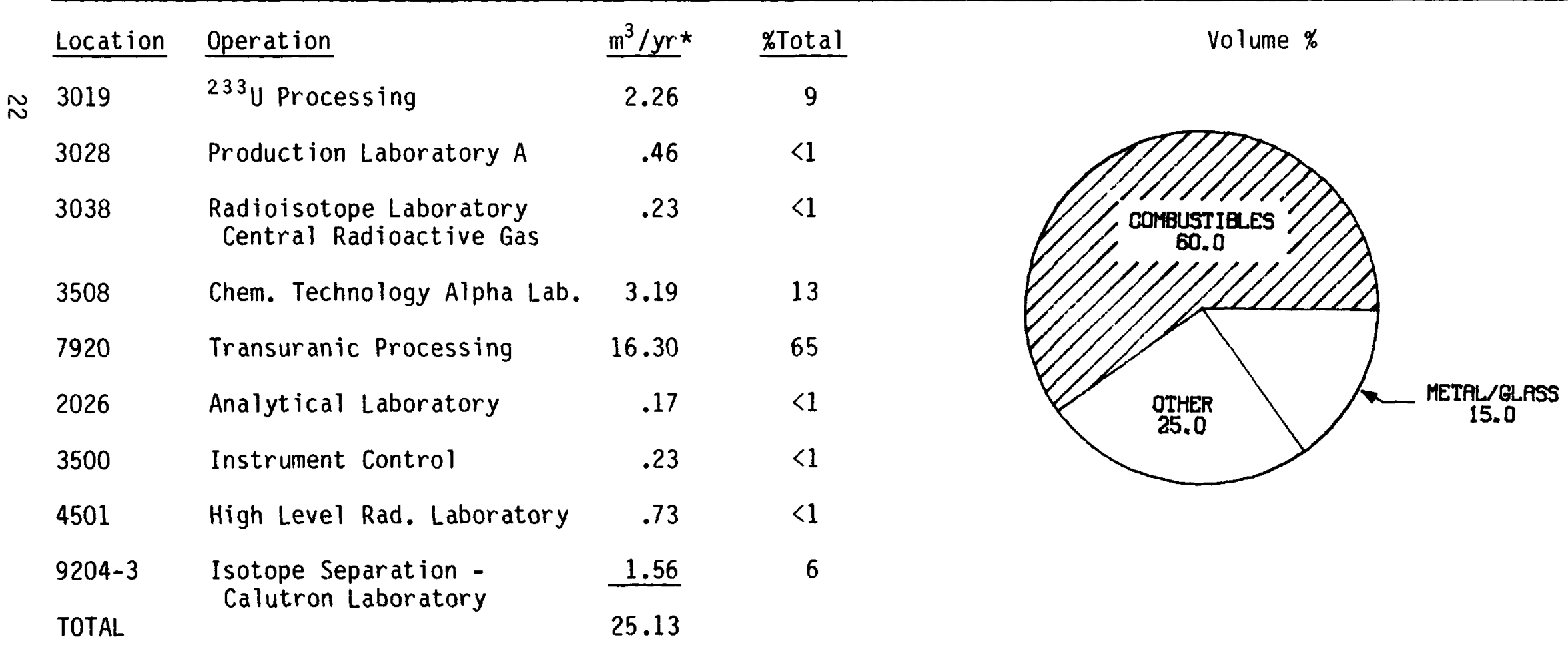

* Based on data from FY 1980 
Table 2.13 Forecast of Transuranic Waste Generation at Rocky Flats Plant ${ }^{3}$ (Contact-Handled)

\begin{tabular}{lcccccc} 
& 1981 & 1982 & 1983 & 1984 & 1985 & $1986-90$ (Average) \\
\cline { 2 - 6 } Volume, $\mathrm{m}^{3}$ & 1650 & 1650 & 1790 & 2050 & 1890 & 1810 \\
Weight, $\mathrm{kg} \times 10^{3}$ & 660 & 660 & 716 & 870 & 756 & 724 \\
Pu content & & & & & & \\
$\quad \mathrm{g}$ & 25,000 & 25,900 & 27,800 & 32,100 & 29,500 & 24,500 \\
$\quad$ curies & 10,600 & 11,000 & 11,800 & 13,600 & 12,500 & 10,400
\end{tabular}

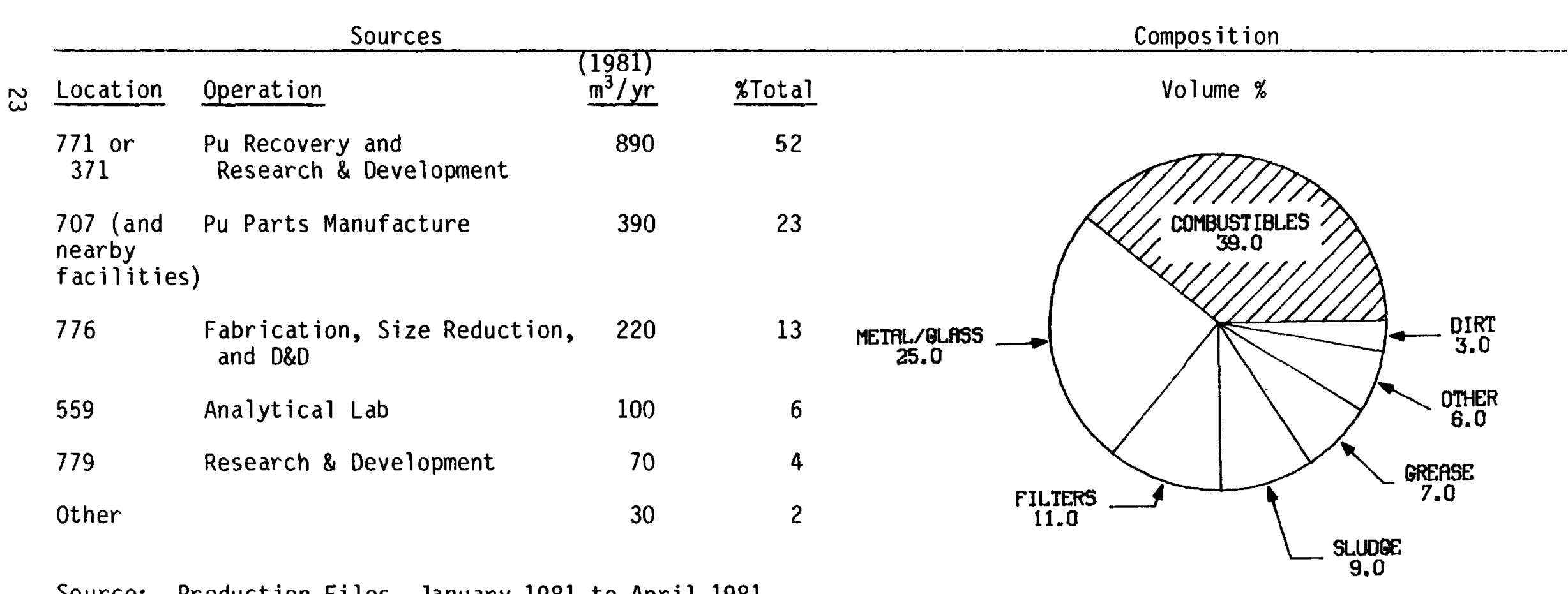


Table 2.14 Forecast of Transuranic Waste Generation at Rockwell Hanford Operations 6,8 (Contact-Handled)

$1981^{(1)}$ 1982 1983 1984 1985 $1986-90$ (Average)

Volume, $\mathrm{m}^{3}$ 808

220

376

410

651 560

Weight, * $\mathrm{kg} \times 10^{3}$

242

66

113

123

195

168

$\mathrm{Pu}$ content**

$\begin{array}{lrrrrrr}g & 18,068 & 5,500 & 9,400 & 10,250 & 16,275 & 14,000 \\ \text { curies*ᄎ* } & 7,660 & 2,332 & 3,986 & 4,346 & 6,901 & 5,936\end{array}$

$\star$ Assumes Avg density $=300 \mathrm{~kg} / \mathrm{m}^{3}$.

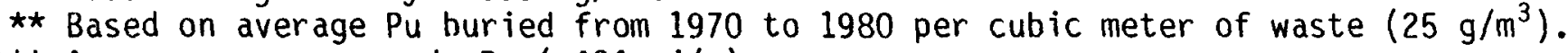

$\star \star \star$ Assumes weapons grade $\mathrm{Pu}(.424 \mathrm{ci} / \mathrm{g})$.

(1) $=1981$ is actual data.

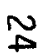

Sources

Composition

\begin{tabular}{|c|c|c|c|}
\hline Location & Current Operation & $\mathrm{m}^{3} / \mathrm{yr}{ }^{*}$ & \%Total \\
\hline $234-52$ & Pu Recovery, R\&D & 330 & $96 \%$ \\
\hline $\begin{array}{l}200 \text { West } \\
\text { Area }\end{array}$ & D\&D projects & 5.6 & $2 \%$ \\
\hline $\begin{array}{l}\text { Tank } \\
\text { Forms }\end{array}$ & $\begin{array}{l}\text { Waste Storage and } \\
\text { Surveillance }\end{array}$ & 7.8 & $2 \%$ \\
\hline TOTAL & & 343.4 & \\
\hline
\end{tabular}

From 1982-85 additional D\&D activities and the start-up of the Purex Recovery Plant will change the locations and volumes a great deal.

* Based on data from 1980.

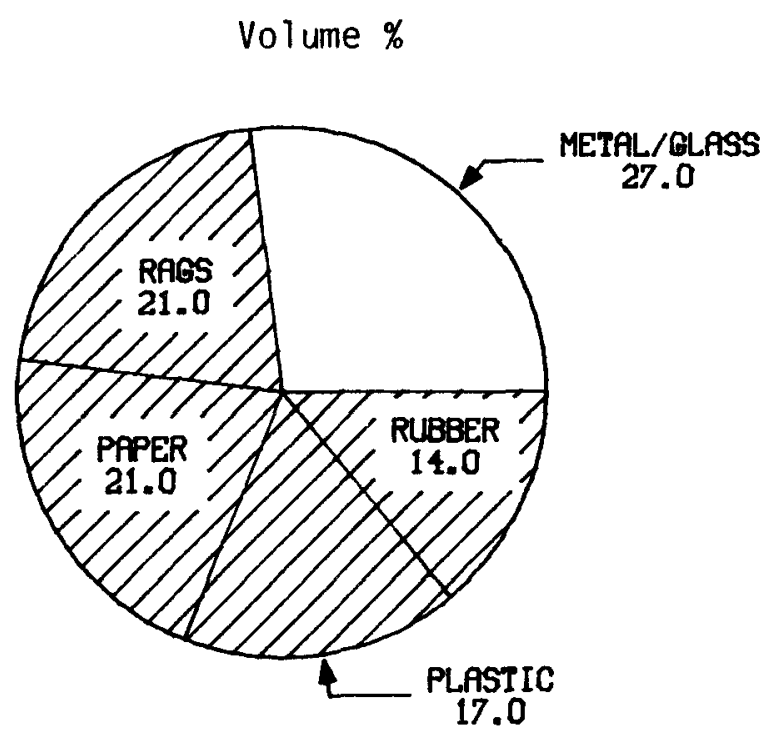


Table 2.15 Forecast of Transuranic Waste Generation at Savannah River Plant and Laboratory 13,14 (Cont act-Handled)

\begin{tabular}{|c|c|c|c|c|c|c|}
\hline & 1981 & 1982 & 1983 & 1984 & 1985 & 1986-90 (Aver age) \\
\hline Volume, $\mathrm{m}^{3}$ & 370 & 445 & 468 & 515 & 552 & 580 \\
\hline Weight*, $\mathrm{kg} \times 10^{3}$ & 111 & 111 & 111 & 108 & 108 & 108 \\
\hline \multicolumn{7}{|l|}{$\mathrm{Pu}$ content } \\
\hline g & 2,759 & 2,756 & 2,766 & 2,769 & 2,773 & 2,770 \\
\hline curies & 35,764 & 36,128 & 36,492 & 36,856 & 37,220 & 37,000 \\
\hline \multicolumn{7}{|c|}{ * Estimated density at $300 \mathrm{~kg} / \mathrm{m}^{3}$. } \\
\hline
\end{tabular}

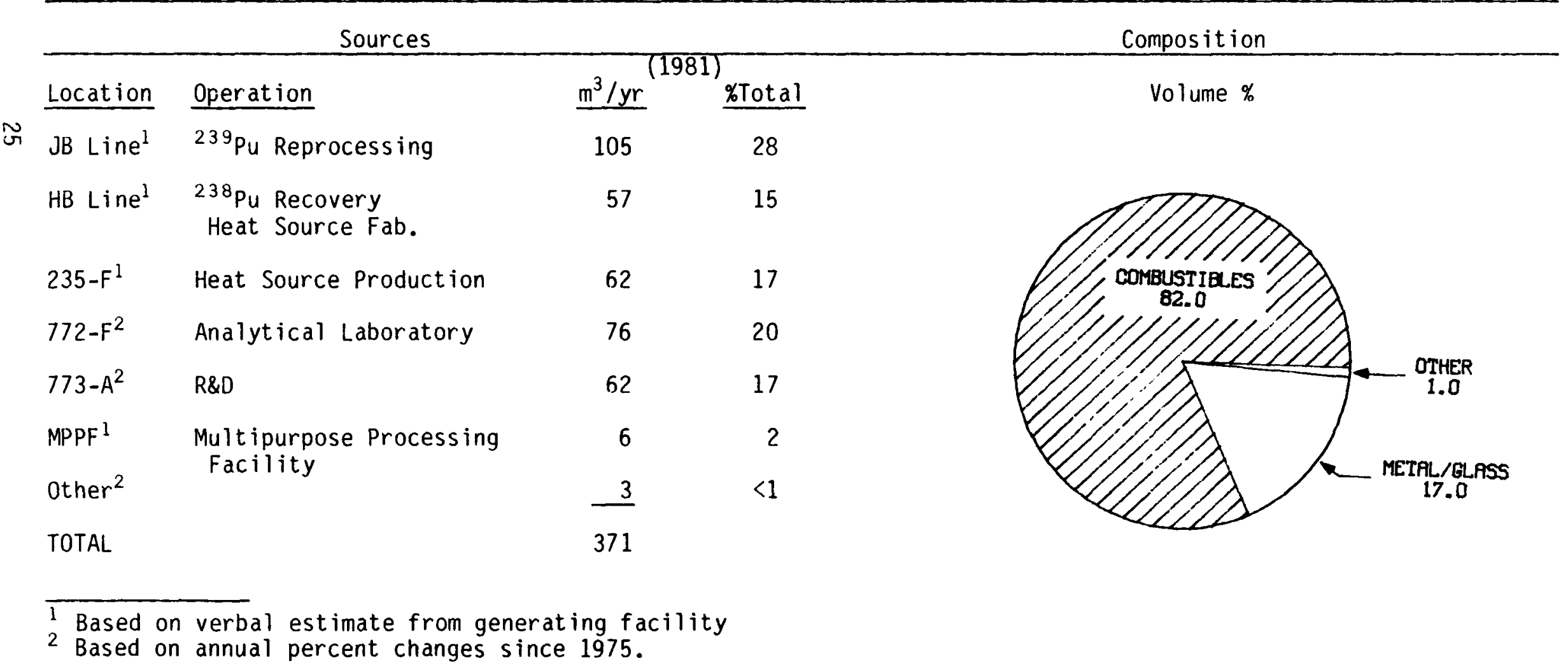


Table 2.16 Forecast of Transuranic Waste Generation at Westinghouse Hanford Company $y^{6,8}$ (Contact-Handled)

\begin{tabular}{|c|c|c|c|c|c|c|}
\hline & 1981 & 1982 & 1983 & 1984 & 1985 & 1986-90 (Average) \\
\hline Volume, $\mathrm{m}^{3}$ & 37 & 506 & 331 & 76 & 142 & 142 \\
\hline Weight, * $\mathrm{kg} \times 10^{3}$ & 14 & 152 & 99 & 23 & 43 & 43 \\
\hline \multicolumn{7}{|l|}{$\mathrm{Pu}$ content } \\
\hline $\mathrm{g}$ & 691 & 1919 & 1919 & 1919 & $N / R$ & $N / R$ \\
\hline curies & 124 & 814 & 814 & 814 & $N / R$ & $N / R$ \\
\hline
\end{tabular}

No

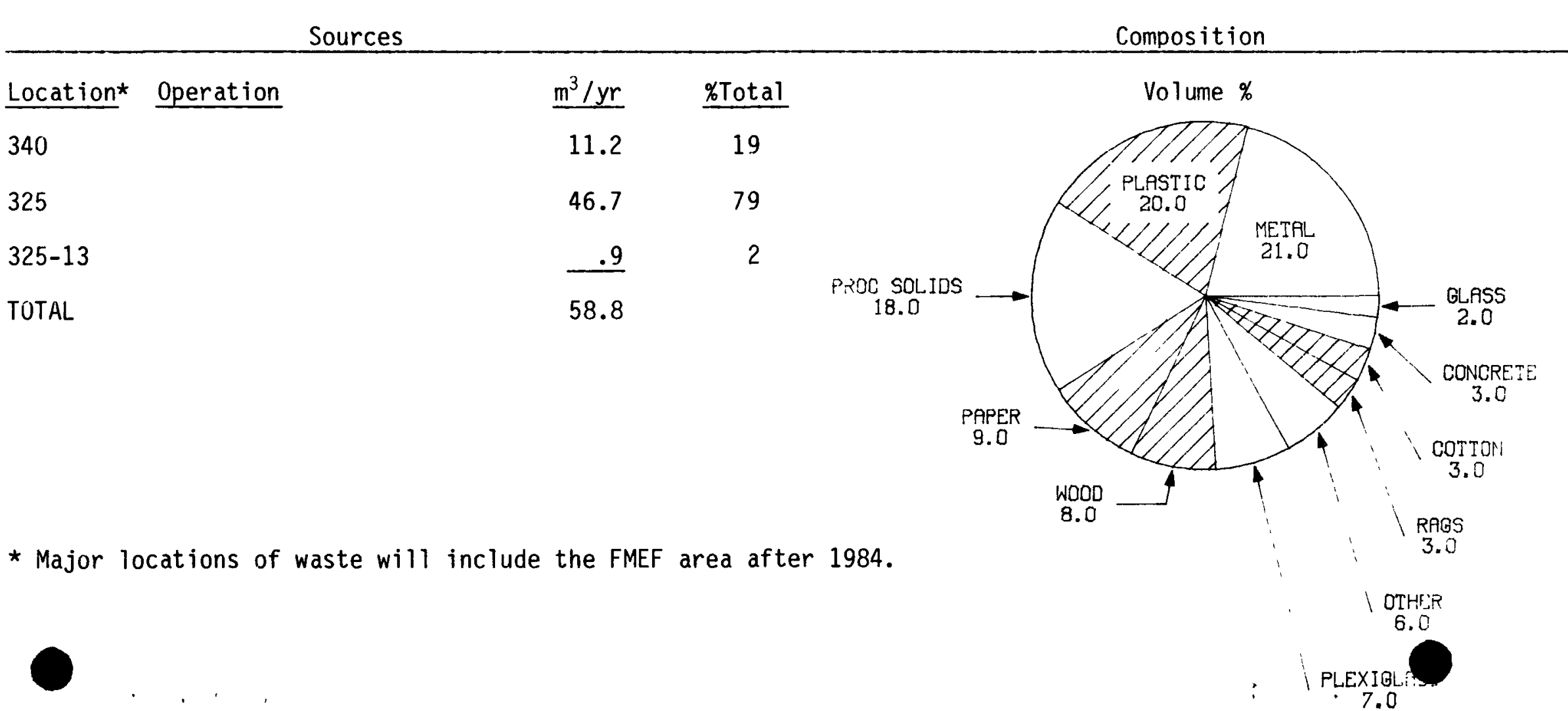


Table 2.17 Forecast of Transuranic Waste Generation at Sites Generating Less Than $25 \mathrm{~m}^{3} / \mathrm{yr}$ (Contact-Handled)

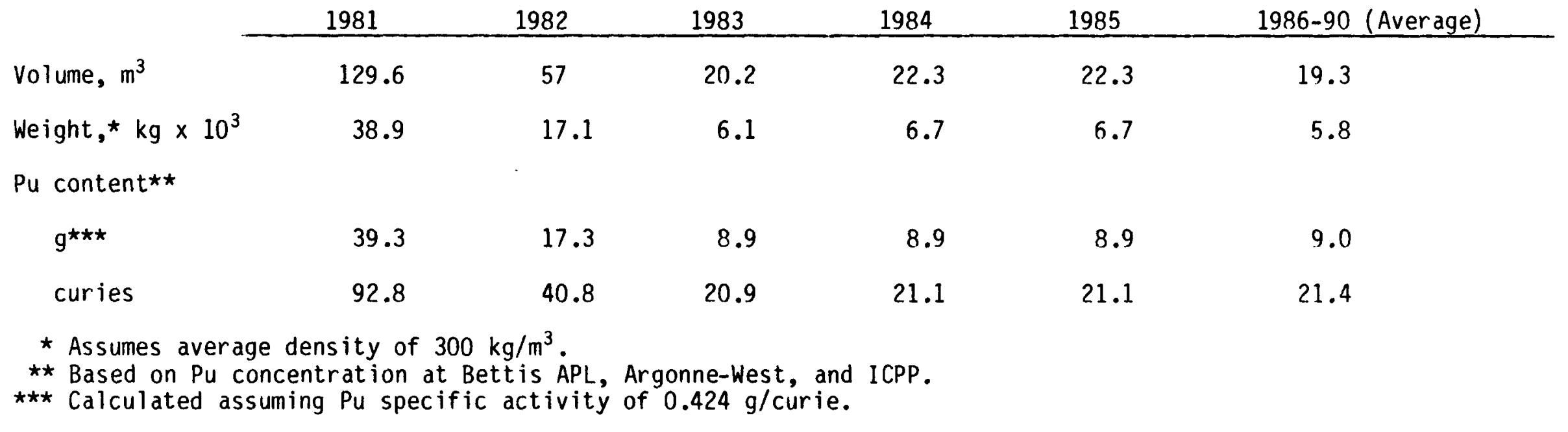

Location

Argonne-West Battelle Columbus* Bett is APL ICPP

INEL Test

Reactor

Lovelace

Sandia

United Nuclear Company

\section{Function}

R\&D; Sodium Research R\&D; Waste is from D\&D R\&D: Reactor Research Chemical Processing Plant Materials \& Fuel

Testing in Nuclear Reactor Medical R\&D

R\&D

Operate Nuclear Reactor

\section{$(10-y r$ ave) $\mathrm{m}^{3} / \mathrm{yr}$}

.$^{.8}$
$34^{\star}$
14.4
$2 \star \star$
.4
2
1
6

Vol \% Ref 3

54
54

7.5

$1.5 \quad 5$

$\begin{array}{ll}7.5 & 1\end{array}$

$\begin{array}{rl}7.5 & 1 \\ 3.7 & 1 \\ 22.6 & 8\end{array}$

Volume \%

Approximately $40 \%$ - combustible $60 \%$ - non-combustible

TOTAL

* Not included in total. Will phase out in 1982.

** Does not include 1981. 


\subsection{Remote-Handled - Summary}

\subsubsection{Locations, Quantities, and Waste Forms}

DOE sites that generate remote-handled TRU waste are listed in Table 2.18. Uncertainties in Research \& Development (R\&D) projects make forecasts of remote-handled waste especially difficult.

A breakdown of the current types of remote-handled waste is also shown in Table 2.18. As with the contact handled waste, this refers to the contents of the containers. The volume, however, includes the container itself. The data contain more detail than contact-handled waste because the percentages come from smaller waste streams. Most of the data are based on estimates from facilities rather than actual storage or burial records. Figure 2.19 presents the totals for all sites. Except for FFTF startup in 1984, very little change is anticipated. Figure 2.20 shows the current percentages of waste forms and containers. Again, these percentages will probably not change until FFTF activities begin. Remote-handled waste forms and their containers are not presently defined for the FFTF project. Because of extensive shielding required, they could be different than present remote-handled waste containers. 
Table 2.18 Projection of Remote Handled TRU Waste Volumes and Waste Forms

\begin{tabular}{|c|c|c|c|c|c|c|c|c|c|c|c|c|c|c|}
\hline & \multicolumn{8}{|c|}{ Volume, $\mathrm{m}^{3}$} & \multicolumn{6}{|c|}{ Volume $x^{c}$} \\
\hline & \multirow[b]{2}{*}{1981} & \multirow[b]{2}{*}{1982} & \multirow[b]{2}{*}{1983} & \multirow[b]{2}{*}{1984} & \multirow[b]{2}{*}{1985} & \multicolumn{2}{|l|}{ Avg/yr } & \multirow[b]{2}{*}{ Cloth } & \multirow[b]{2}{*}{ Plastic } & \multirow[b]{2}{*}{ Rubber } & \multirow[b]{2}{*}{ Glass } & \multirow[b]{2}{*}{ Metal } & \multirow[b]{2}{*}{ Other } & \multirow[b]{2}{*}{ Ref. } \\
\hline & & & & & & 1986-90 & Paper & & & & & & & \\
\hline Argonne - East & 21 & 7 & 7 & 7 & 7 & 7 & $17 \%$ & * & $16 \%$ & $16 \%$ & $13 \%$ & $25 \%$ & $13 \%$ & 4 \\
\hline Argonne - West & .8 & .75 & 1.0 & 1.0 & 1.0 & 1.0 & $50 \%$ & - & \multicolumn{2}{|c|}{ combustibles } & $0 \%$ & $50 \%$ & $0 \%$ & 5 \\
\hline IOPP & .3 & 7 & 14 & 14 & 14 & 14 & $0 \%$ & $0 \$$ & $0 \%$ & 08 & $0 \$$ & $0 \%$ & $\mathbf{a}$ & 5 \\
\hline $\begin{array}{l}\text { INEL Naval Reactor } \\
\text { Facilitty }\end{array}$ & 1 & 1.36 & 1.4 & 2.0 & 2.0 & 1.4 & $100 \%$ & \multicolumn{3}{|c|}{ combustibles } & $0 \%$ & $0 \not$ & b & 16 \\
\hline $\begin{array}{c}\text { Los Alamos Nat. } \\
\text { Laboratory }\end{array}$ & 10.6 & 5 & 5 & 5 & 5 & 5 & $9 \%$ & * & $39 \%$ & 38 & 88 & $23 \%$ & $18 \%$ & 9,17 \\
\hline $\begin{array}{c}\text { Oak Ridge Nat. } \\
\text { Laboratory }\end{array}$ & 26.8 & 34.3 & 34.3 & 34.3 & 34.3 & 57 & $38 \%$ & $3 \%$ & 258 & 258 & * & $10 \%$ & $0 \%$ & 12,18 \\
\hline $\begin{array}{l}\text { Westinghouse Hanford } \\
\text { Company }\end{array}$ & 1 & 2 & 2.7 & 23 & 45 & 45 & 178 & 258 & $41 \%$ & $0 \%$ & 38 & $14 \%$ & $0 \%$ & 6,8 \\
\hline $\begin{array}{l}\text { * Not avallable } \\
\text { a Nearly } 100 \% \text { of ICPf } \\
\text { b Nearly } 100 \% \text { of was } \\
\text { c NOTE: Because the } \\
\text { container of waste } \\
\text { this latter value. }\end{array}$ & $\begin{array}{l}\text { waste } \\
\text { e is } \\
\text { actual } \\
\text { only a }\end{array}$ & $\begin{array}{l}\text { comes } \\
\text { ombust } \\
\text { waste } \\
\text { sma I I }\end{array}$ & $\begin{array}{l}\text { from } \\
\text { ible, } \\
\text { is pl } \\
\text { fract }\end{array}$ & $\begin{array}{l}\text { fllte } \\
\text { appro } \\
\text { aced } \\
\text { rion }\end{array}$ & $\begin{array}{l}\text { S. } \\
\text { imatel } \\
\text { n heav } \\
-10 \%)\end{array}$ & $\begin{array}{l}5 \mathrm{~g} \text { in } \\
\text { Iy shiel } \\
\text { s actua }\end{array}$ & $\begin{array}{l}\text { very } 7 \\
\text { d cask } \\
y \text { gener }\end{array}$ & $\begin{array}{l}\text { cublc } \\
\text { s whicl } \\
\text { ated wa }\end{array}$ & $\begin{array}{l}\text { feet consi } \\
\text { h become } p \\
\text { aste. The }\end{array}$ & $\begin{array}{l}\text { ists of } \\
\text { art of } \\
\text { percen }\end{array}$ & $\begin{array}{l}\text { ctual f } \\
\text { he wast } \\
\text { compos }\end{array}$ & $\begin{array}{l}\text { uel. } \\
e, \text { for } \\
\text { ition }\end{array}$ & $\begin{array}{l}\text { very } \\
\text { fers }\end{array}$ & 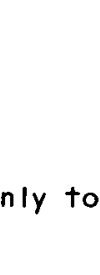 \\
\hline
\end{tabular}




\subsection{Review of Major Sites that Generate Remote-Handled Waste}

Tables 2.21 through 2.25 present the locations and sources for all remote-handled TRU waste generation. The information shown in these tables is presented in the same format as for contact-handled waste. 
Table 2.21 Forecast of Transuranic Waste Generation at Argonne National Laboratory - East ${ }^{4}, 5$ (Remote-Hand led)

\begin{tabular}{|c|c|c|c|c|c|c|}
\hline & 1981 & 1982 & 1983 & 1984 & 1985 & 1986-90 (Average) \\
\hline Volume, $\mathrm{m}^{3}$ & 15 & 10 & 10 & 10 & 10 & 10 \\
\hline Weight*, $\mathrm{kg} \times 10^{3}$ & 5.8 & 3.9 & 3.9 & 3.9 & 3.9 & 3.9 \\
\hline \multicolumn{7}{|l|}{ Pu content } \\
\hline$g$ & 46.4 & 31 & 31 & 31 & 31 & 31 \\
\hline curies & 3.6 & 2.4 & 2.4 & 2.4 & 2.4 & 2.4 \\
\hline
\end{tabular}

* Based on density of alpha hot cell waste sent to INEL $\left(388 \mathrm{~kg} / \mathrm{m}^{3}\right)$.

$\omega$

$\begin{array}{ll}\text { Location } & \text { Operation } \\ \text { Bldg. 212* } & \text { Research on } \\ \text { (Materials } & \text { Special Nuclear } \\ \text { Science } & \text { Materials } \\ \text { Division) } & \end{array}$

* Responsible for over $99 \%$ of Argonne Remote-Handled Waste

\section{Sources}

$\underline{m^{3} / y r}$

10

(Materials

Division)

\section{Composition}

Volume $\%$

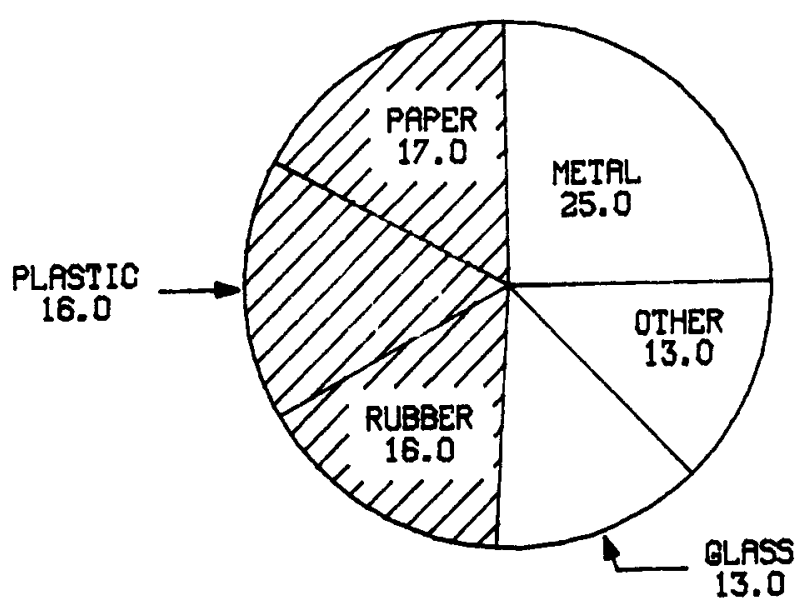


Table 2.22 Forecast of Transuranic Waste Generation at Idaho National Engineering Laboratory ${ }^{5} 16$ (Remote-Handled)

\begin{tabular}{|c|c|c|c|c|c|c|}
\hline & 1981 & 1982 & 1983 & 1984 & 1985 & 1986-90 (Aver age) \\
\hline Volume, $\mathrm{m}^{3}$ & 2.1 & 9.1 & 16.4 & 17 & 17 & 16.4 \\
\hline Weight*, $\mathrm{kg} \times 10^{3}$ & .8 & 3.5 & 6.2 & 6.5 & 6.5 & 6.2 \\
\hline $\mathrm{Pu}$ content** & & & & & & \\
\hline $\mathrm{g}$ & 77.7 & 336.7 & 606.8 & 629 & 629 & 606.8 \\
\hline curies & $N / A$ & $N / A$ & $N / A$ & $N / A$ & $N / A$ & $N / A$ \\
\hline
\end{tabular}

* Based on estimated density of INEL waste codes $151,152,153,154,155\left(380 \mathrm{~kg} / \mathrm{m}^{3}\right)$.

$\star \star$ Based on estimated Pu density of same codes $\left(37 \mathrm{~g} / \mathrm{m}^{3}\right)$.

N/A Not available.

Sources

Composition

\begin{tabular}{|c|c|c|c|}
\hline Location & Operation & $\mathrm{m}^{3} / \mathrm{yr}$ (avg) & Vol $\%$ \\
\hline Argonne-West & Fuels Testing & 1 & $7 \%$ \\
\hline ICPP & Chemical Processing & 12 & $83 \%$ \\
\hline $\begin{array}{l}\text { INEL Naval } \\
\text { Reactor Facility }\end{array}$ & Fuels Testing & 1.5 & $10 \%$ \\
\hline
\end{tabular}

TOTAL

14.5

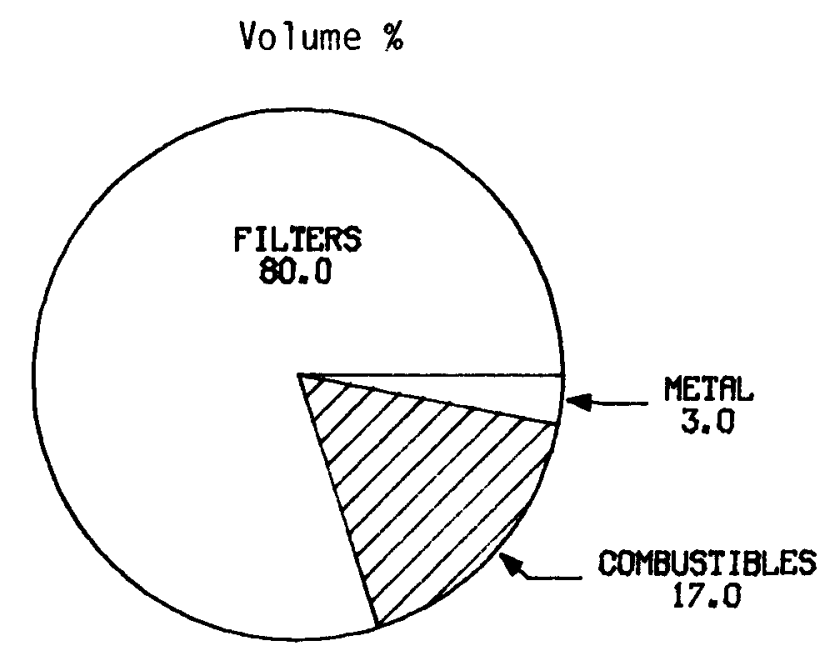


Table 2.23 Forecast of Transuranic Waste Generation at Los Alamos National Laboratory ${ }^{9}, 10$ (Remote-Hand led)

\begin{tabular}{lcccccc} 
& 1981 & 1982 & 1983 & 1984 & 1985 & $1986-90$ (Average) \\
\cline { 2 - 7 } Volume, $\mathrm{m}^{3}$ & 10.6 & 5 & 5 & 5 & 5 & 5 \\
Weight, $\mathrm{kg} \times 10^{3}$ & 17 & 8 & 8 & 8 & 8 & 8 \\
Pu content & & & & & & \\
$\mathrm{g}$ & 400 & 400 & 400 & 400 & 400 & 400 \\
$\quad$ curies* & 740 & 750 & 750 & 750 & 750 & 750
\end{tabular}

* Including Mixed Fission Product content.

Sources

w Location

TA-3-29

Operation

Chemical and

Metallurgical

Research
Composition

$\frac{\mathrm{m}^{3} / \mathrm{yr}}{5}$

Volume $\%$

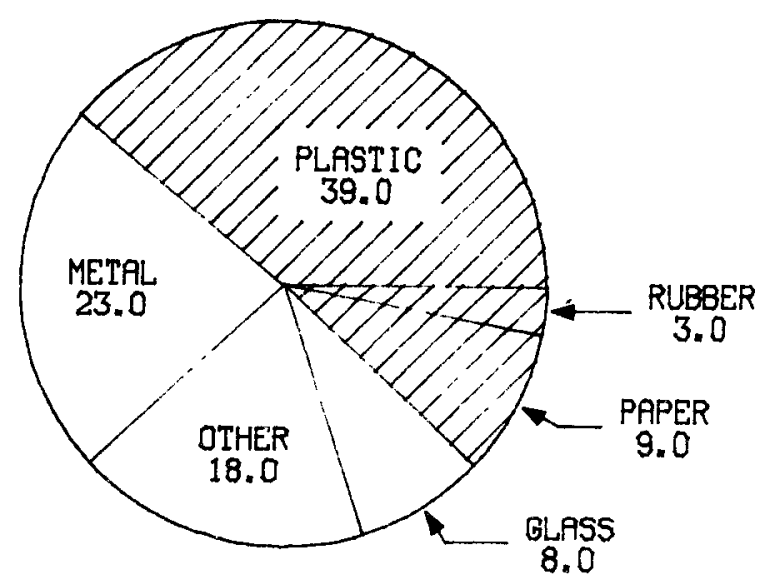


Table 2.24 Forecast of Transuranic Waste Generation at Oak Ridge National Laboratory ${ }^{12}, 18$ (Remote-Handled)

\begin{tabular}{lcccccc} 
& 1981 & 1982 & 1983 & 1984 & 1985 & $1986-90($ \\
\cline { 2 - 7 } Volume, $\mathrm{m}^{3}$ & 34.3 & 34.3 & 34.3 & 34.3 & 34.3 & 34.3 \\
Weight*, $\mathrm{kg} \times 10^{3}$ & 61.7 & 61.7 & 61.7 & 61.7 & 61.7 & 61.7 \\
TRU content & & & & & & \\
$\quad \mathrm{g}$ & 15.2 & 15.2 & 15.2 & 15.2 & 15.2 & 15.2 \\
$\quad$ curies & 95.9 & 95.9 & 95.9 & 95.9 & 95.9 & 95.9
\end{tabular}

* Based on density of beta-gamma TRU waste in 3rd Quarter 1981 ORNL Waste Disposal Log $\left(1800 \mathrm{~kg} / \mathrm{m}^{3}\right)$.

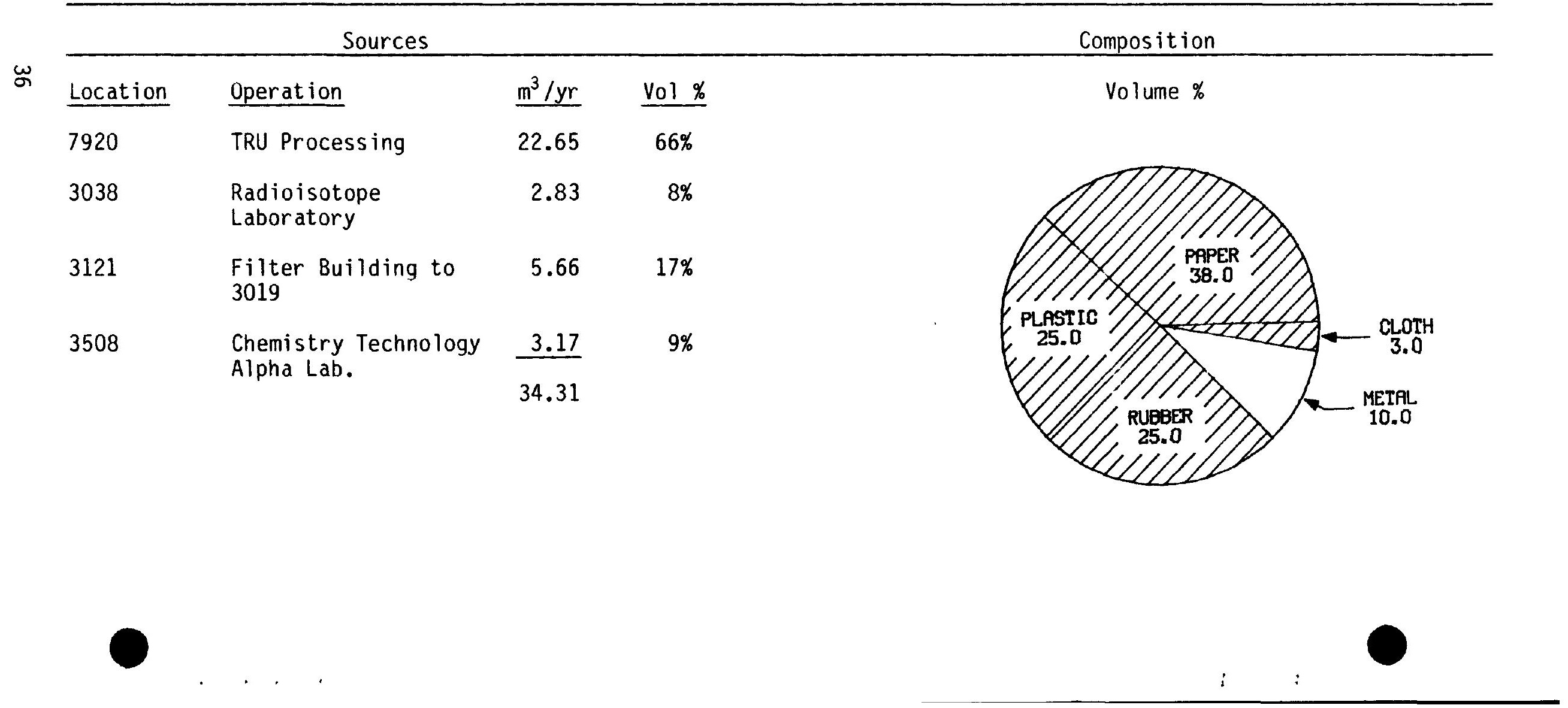


Table 2.25 Forecast of Transuranic Waste Generation at Westinghouse Hanford Company 6.8 (Remote-Handled)

\begin{tabular}{|c|c|c|c|c|c|c|}
\hline & $1981 \star \star \star \star$ & 1982 & 1983 & 1984 & 1985 & 1986-90 (Aver age) \\
\hline Volume, $\mathrm{m}^{3}$ & 1 & 2 & 2.7 & 23 & 45 & 45 \\
\hline Weight*, $\mathrm{kg} \times 10^{3}$ & .77 & 1.74 & 2.3 & $\star \star$ & ** & $\star \star$ \\
\hline \multicolumn{7}{|l|}{ Pu content } \\
\hline$g$ & 5 & $N / R$ & $N / R$ & $N / R$ & $N / R$ & $N / R$ \\
\hline curies & 1.9 & $N / R$ & $N / R$ & $N / R$ & $N / R$ & $N / R$ \\
\hline
\end{tabular}

$\underline{w}$

\begin{tabular}{cc}
\hline Sources & Composition \\
\hline
\end{tabular}

$\begin{array}{llcc}\text { Location } & \text { Operation } & \begin{array}{c}(1981-83) \\ \mathrm{m}^{3} / \mathrm{yr}\end{array} & \text { Vol\% } \\ \begin{array}{ll}327-\mathrm{C} \\ \text { Building }\end{array} & \begin{array}{l}\text { Research on Nuclear } \\ \text { Reactor Fuels }\end{array} & 5 & 100^{\star} \\ \text { FMEF } & \begin{array}{l}\text { Fuels \& Material } \\ \text { Examination }\end{array} & 38^{\star} & \end{array}$

* FMEF begins operation 1984, and is not included in total above

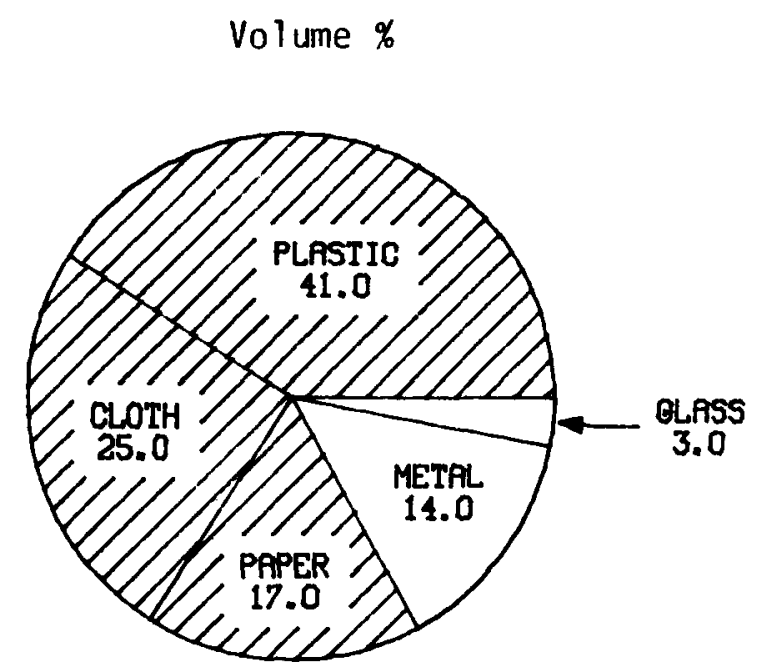




\section{CHARACTERISTICS OF STORED TRU WASTES}

\subsection{Contact-Handled - Summary}

\subsubsection{Locations, Quantities, and Waste Forms}

TRU wastes are currently stored at six DOE sites. These sites are shown geographically in Figure 1.1. Figure 3.2 indicates how much waste was in storage as of December 31, 1980, and it projects how much will be added during the next 10 years. This projection is based on the forecasts from the Newly Generated Waste section of this report. Stored waste is found in many different types of storage and containers. These will be covered in detail in Section 3.2 .

Table 3.1 shows the total amount of stored waste at each site as of December 31, 1980, and the amount of each waste form. Data were tabulated from Tables 3.4 through 3.11. The data are limited in scope because records are not readily available at some sites. At Hanford, ORNL, and SRP, the records usually distinguish only between combustible and noncombustible, and these data are known to be questionable. By going to the production facilities, an approximation of the physical characteristics was obtained for the current waste streams. These typical percentages were applied to the total volumes, which resulted in an estimated composition of the total waste in storage. When actual data were available (INEL and LANL) they were used. Other sites provided estimates based on the best records available, which were often incomplete. In cases where the most recent year's percentage was used, different processes or events may have changed waste forms, but for the period 1970 to 1980 it is thought to be reasonably accurate. Combining the data from all sites is a difficult task because different categories have a wide assortment of waste forms. For example, waste at the Idaho National Engineering Laboratory storage site includes well over 100 categories of waste. However, some categories--such as combustibles--may include plastic wrap, cloth gloves, wood, paper, and 
Table 3.1 Contact Handled Waste Forms at TRU Storage Sites (Vol. \%)

\begin{tabular}{|c|c|c|c|c|c|c|c|c|}
\hline Site & Combustibles & Metal & Sludge(1) & $\begin{array}{l}\text { Concrete/(2) } \\
\text { Sludge }\end{array}$ & $\begin{array}{l}\text { Filters \& } \\
\text { Filter } \\
\text { iledia }\end{array}$ & $\begin{array}{l}\text { Dirt, } \\
\text { Gravel, } \\
\text { Asphalt }\end{array}$ & Glass & other \\
\hline Hanford (3) & 67 & 26 & 2.5 & .5 & $\begin{array}{l}\text { Not } \\
\text { Reported }\end{array}$ & .5 & a & 3.5 \\
\hline $\operatorname{INEL}^{(4)}$ & 19 & 32 & 8 & 9 & 7 & 2 & 2 & 21 \\
\hline $\operatorname{LANL}^{(4)}$ & 12 & 51 & 5 & 15 & 3 & 2 & $\mathrm{a}$ & $1 ?$ \\
\hline NTS $(4)$ & 89 & 4 & 4 & 0 & b & 2 & $\mathrm{a}$ & 0 \\
\hline $\mathrm{ORNL}^{(5)}$ & 55 & 15 & $N / A$ & $N / A$ & $N / A$ & $N / A$ & $N / A$ & 30 \\
\hline $\operatorname{SRP}(3)$ & 82 & 9 & 0 & 0 & $a, b$ & 0 & 8.5 & .5 \\
\hline
\end{tabular}

(1) Includes organic and inorganic process solids, liquid waste solidified with various sorbents, and dryed salts.

(2) Includes concrete, (some asphalt), and process waste which has been immobilized with concrete.

(3) Based on limited sampling of waste during 1981 only. (Ref. 6, 13).

(4) Based on records received from generator or site records since 1971. (Ref. 19, 9, 20).

(5) Data not available except for very general categories. (Ref. 12).

a Included with metal.

b Included with combustibles. 
Figure 3.2

\section{CONTACT HANDLED WASTE FORMS AND WASTE CONTAINERS IN STORAGE TO DECEMBER 31, 1980}

INVENTORY TO DECEMBER 31, 1980 VOLUME \%

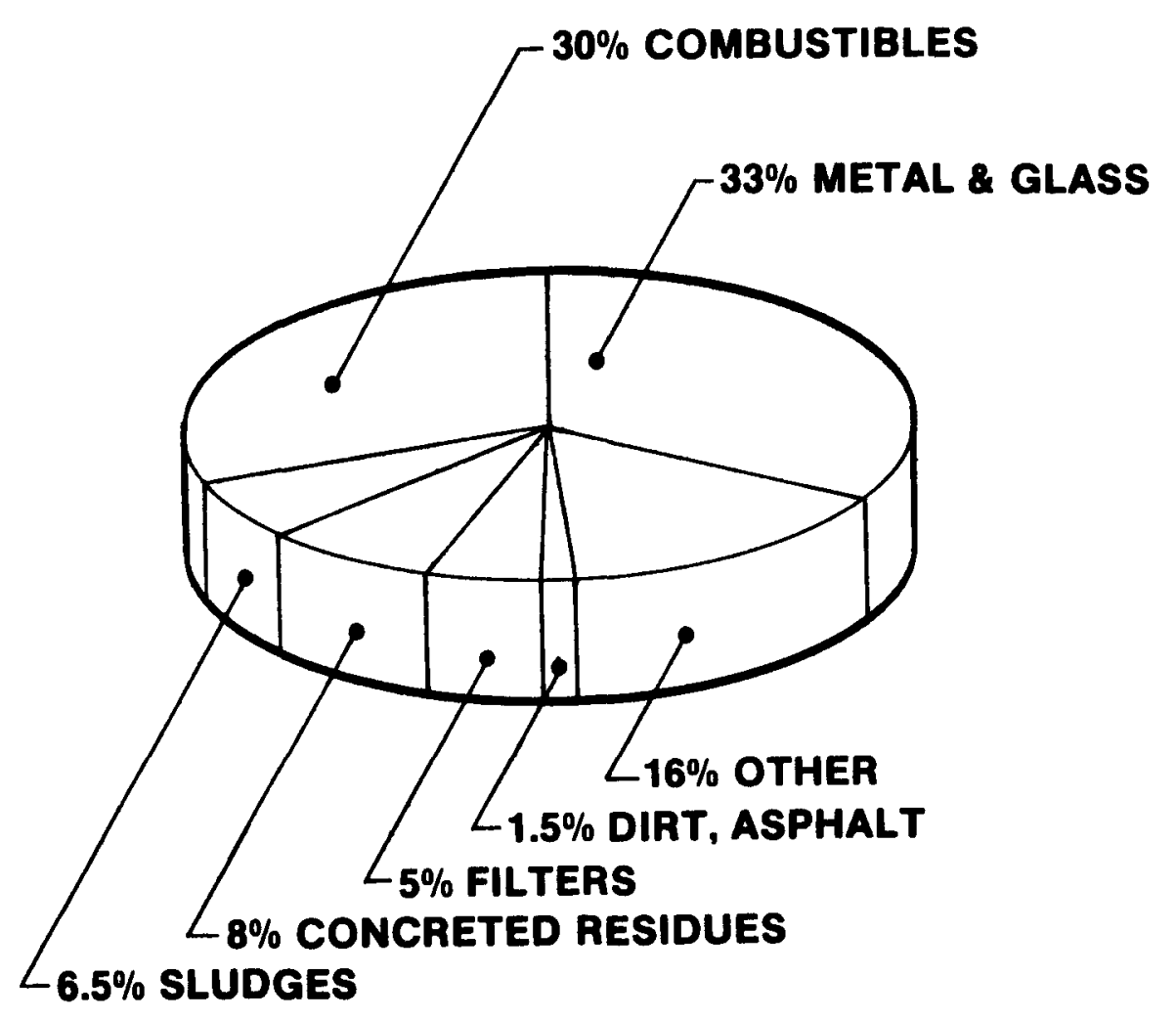

INVENTORY TO DECEMBER 31, 1980 VOLUME \%

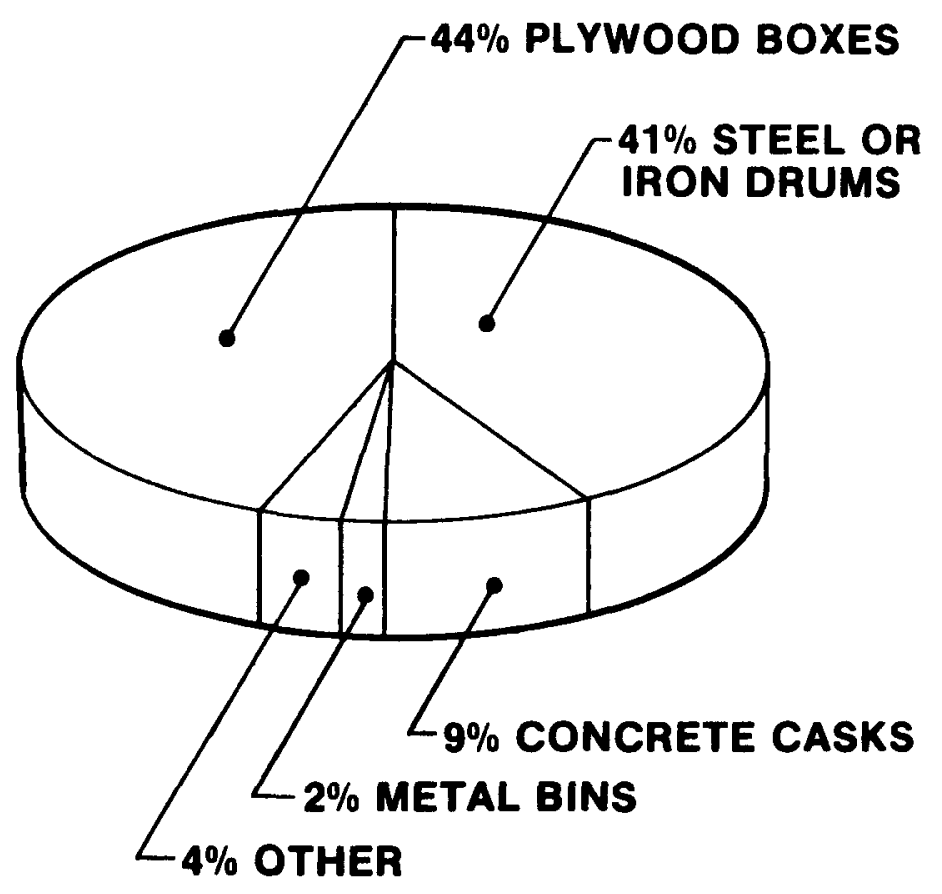

$\left(\right.$ TOTAL VOLUME $\left.=60,064 \mathrm{~m}^{3}\right)$ 


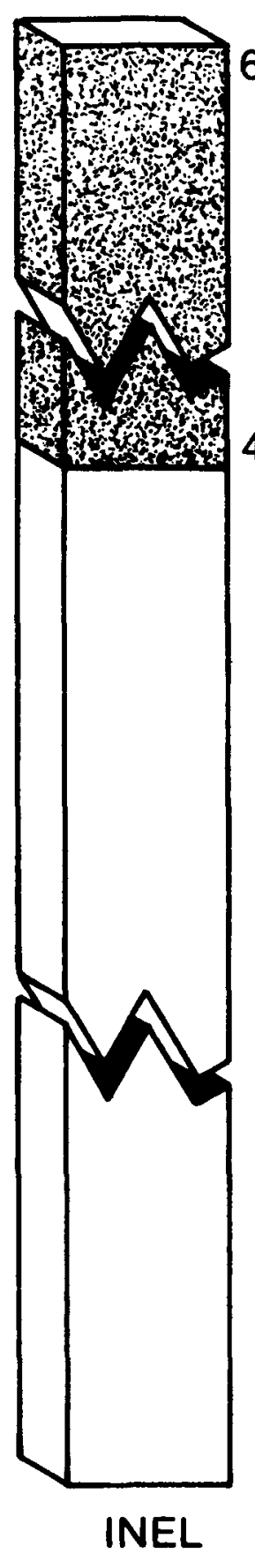

$68,278 \mathrm{~m}^{3}$

\section{Figure 3.3 \\ CURRENT AND PROJECTED INVENTORIES OF STORED TRU WASTE AT DOE SITES}

\section{$42,509 \mathrm{~m}^{3}$}

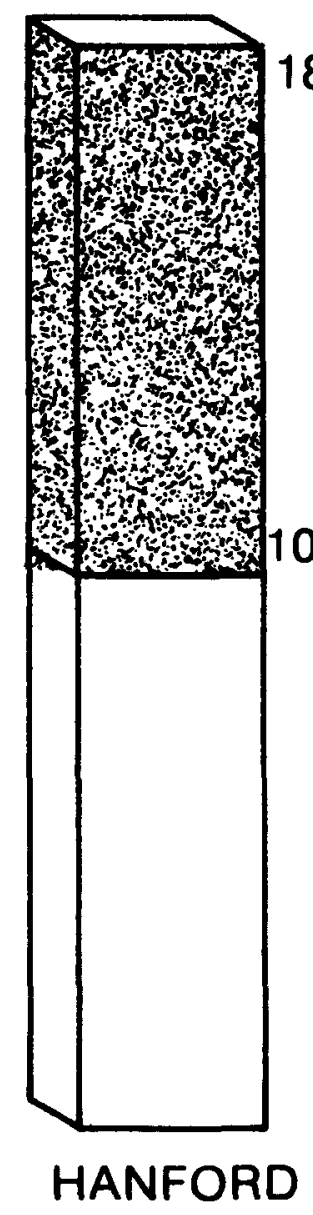

$18,558 \mathrm{~m}^{3}$

BSG TRU WASTE TODEC. 1990

$\square$ TRU WASTE TO DEC. 1980

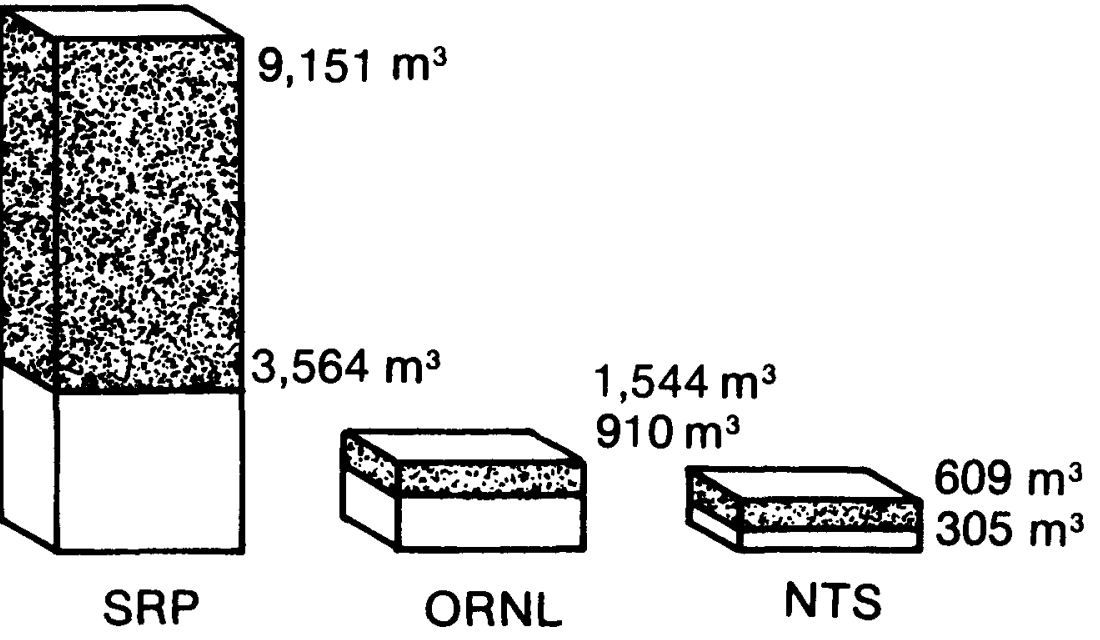


rubber. Hanford, on the other hand, does not categorize various kinds of equipment, metals, sludges, and filters, but they did segregate all combustible waste forms such as paper, cloth, and plastic. The categories in Table 3.1 were defined as clearly as possible, but some overlapping does exist. Even on those sites that kept extensive records, examination of some of the drums indicates that the records do not always match the contents. ${ }^{23}$ Figure 3.3 shows the total percentages of the various waste forms from Table 3.1. It also shows how these waste forms are currently packaged. These data are based on the detailed information found in Section 3.2 .

One other aspect of physical waste forms is often overlooked. All records of waste forms are based upon the composition of the waste "inside" the container. However, except for the Hanford site, the volume of waste reported is always based on the entire container volume. ${ }^{8}$ Therefore, a volume of waste may be labeled as combustible, but that volume would also include the steel waste drum which is not combustible. Table 3.4 shows the percentages of waste forms based on the waste inside the container. It also shows the percentages of the waste forms when the volume of the individual containers are added to the categories in which they fall. For example, a 55-gallon drum is usually considered to equate to $0.217 \mathrm{~m}^{3}$ of waste. The drum itself occupjes $0.0083 \mathrm{~m}^{3}$. If the drum contains combustible material, it would be reported as $0.217 \mathrm{~m}^{3}$ of combustibles. In reality, there are $0.209 \mathrm{~m}^{3}$ or less of combustibles, and $0.0083 \mathrm{~m}^{3}$ of steel. In most cases there is less than $0.209 \mathrm{~m}^{3}$ because rarely are drums packed to capacity. Calculations in Table 3.4 are based on the total number of containers found in Section 2.2 .

\subsubsection{Transuranic Content and Surface Dose Rate}

Table 3.5 lists the common isotopes reported by the various storage sites. Other TRU isotopes are found in small quantity in the waste, but they were not researched. They include ${ }^{242} \mathrm{Pu},{ }^{244} \mathrm{Pu},{ }^{242} \mathrm{Am},{ }^{243} \mathrm{Am}$, six isotopes of curium, four isotopes of californium, and ${ }^{247} \mathrm{BK}$. As 
Table 3.4

Effect of Container Volume on Waste Form Volume

\begin{tabular}{|c|c|c|c|c|}
\hline \multirow[b]{2}{*}{ Waste Forms } & \multicolumn{2}{|c|}{$\begin{array}{c}\text { Container Volume } \\
\text { Included With } \\
\text { Contents }\end{array}$} & \multicolumn{2}{|c|}{$\begin{array}{c}\text { Container Volume } \\
\text { Separated Into } \\
\text { Own Category* }\end{array}$} \\
\hline & $\begin{array}{c}\begin{array}{l}\text { Volume } \\
\left(\mathrm{m}^{3}\right)\end{array} \\
\end{array}$ & $\begin{array}{l}\text { Volume } \\
(\%) \\
\end{array}$ & $\begin{array}{c}\text { Volume } \\
\left(\mathrm{m}^{3}\right)\end{array}$ & $\begin{array}{l}\text { Volume } \\
(\%)\end{array}$ \\
\hline Combustibles & 18,385 & 30.5 & 19,917 & 33 \\
\hline Metal & 19,124 & 31.5 & 18,120 & 30 \\
\hline Filters - Filter Media & 3,024 & 5 & 2,754 & 4.5 \\
\hline Dirt, Gravel, Asphalt & 829 & 1.5 & 782 & 1.5 \\
\hline Concrete or Cemented Waste & 4,646 & 7.5 & 4,872 & 8 \\
\hline Absorbed Liquids or Processed Sludges & 3,939 & 6.5 & 8,808 & 5 \\
\hline Glass & 914 & 1.5 & 905 & 1.5 \\
\hline other & 9,574 & 16 & 9,277 & 15.5 \\
\hline & 60,435 & & 60,435 & \\
\hline
\end{tabular}

ऋ The volume of each container has been subtracted from the contents and added to its appropriate category. For instance, the volume of a 55-gallon drum is added to metal category. 
Table 3.5 Major TRU Radionuclide Content in Stored Wastes (To December 31, 1980)

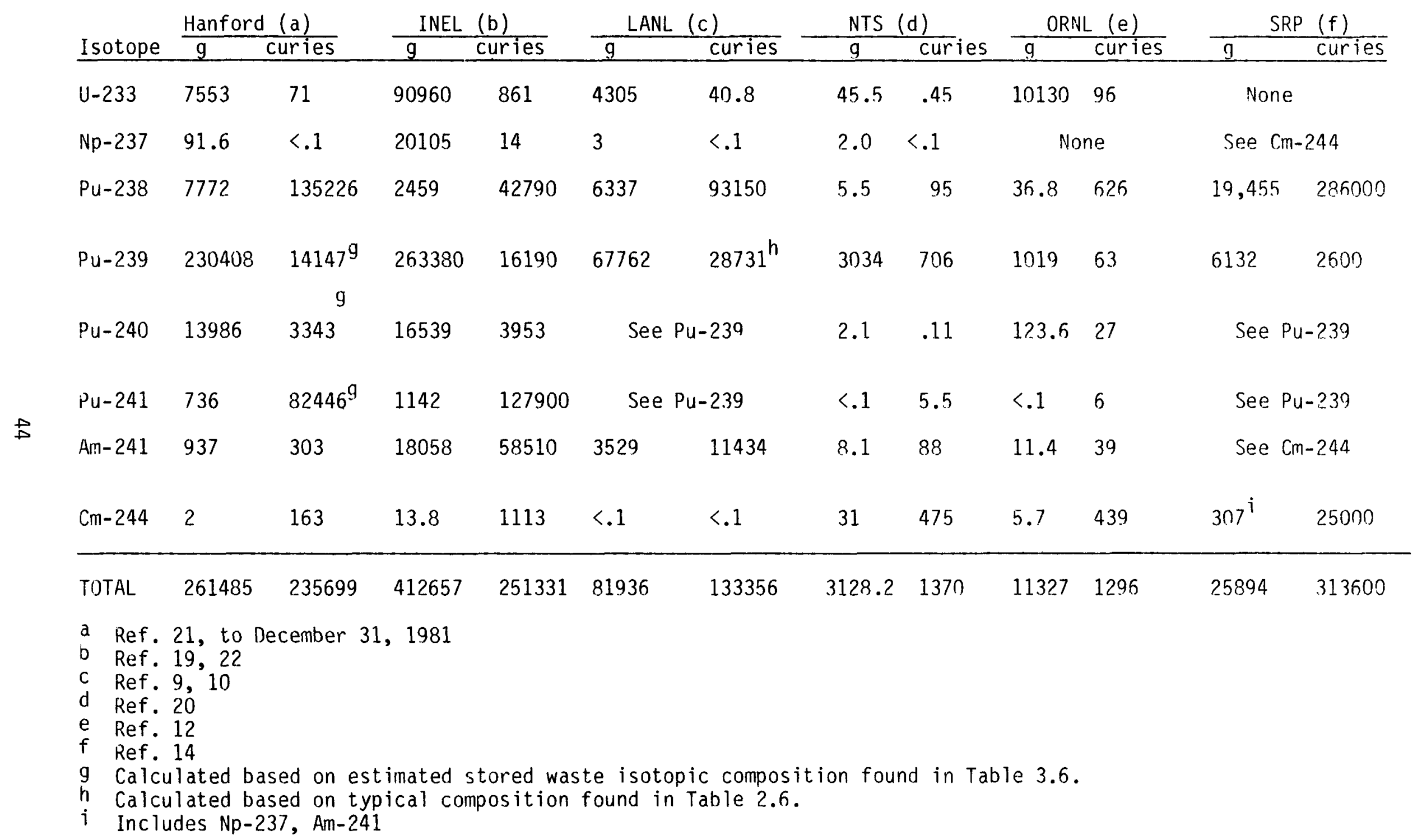


mentioned earlier, the isotopic fraction found in any volume of waste is dependent upon the process that generated the waste. It is sometimes convenient for estimating purposes to know the overall percentages of the major TRU isotopes present at each site. Table 3.6 shows such an estimate. These data are not to be taken as the isotopic composition for each package of waste. It is a composite estimate which can be used when nothing else is available to describe the isotopes. Individual containers of waste may vary widely from the table. For instance, the ORNL remote-handled waste shows 0.01 percent ${ }^{252} \mathrm{Cf}$. In one facility no ${ }^{252} \mathrm{Cf}$ is present, therefore, waste from that facility does not contain 0.01 percent ${ }^{252} \mathrm{Cf}$. At another facility, however, ${ }^{252} \mathrm{Cf}$ is produced, and waste from that facility will contain ${ }^{252} \mathrm{Cf}$ at a much greater amount than 0.01 percent. In most cases detailed compositions were not readily available. Many sites do have this type of data on file but there was not enough time to analyze it. 
Table 3.6

Estimated TRIJ Isotopes in Buried and Stored Waste

CH Stored Waste $\mathrm{RH}$ Stored Waste Buried

Site (Isotope) (wt \%) (Isotope) (wt \%) (Isotope) (wt \%) Ref.

\begin{tabular}{|c|c|c|c|c|c|}
\hline \multirow[t]{6}{*}{ Hanford } & $2{ }^{38} \mathrm{Pu}$ & 0.03 & ${ }^{238} \mathrm{Pu}$ & 0.03 & Not Available \\
\hline & ${ }^{239} \mathrm{Pu}$ & 93.9 & ${ }^{239} \mathrm{Pu}$ & 87.6 & \\
\hline & ${ }^{240} \mathrm{Pu}$ & 5.7 & ${ }^{240} \mathrm{Pu}$ & 12.0 & \\
\hline & ${ }^{241} \mathrm{Pu}$ & 0.3 & ${ }^{241} \mathrm{Pu}$ & 0.3 & \\
\hline & ${ }^{242} \mathrm{Pu}$ & 0.03 & ${ }^{242} \mathrm{Pu}$ & 0.03 & \\
\hline & ${ }^{241} \mathrm{Am}$ & 0.02 & ${ }^{241} \mathrm{Am}$ & 0.02 & \\
\hline
\end{tabular}

INEL

$\begin{array}{lccrrrr}233 \mathrm{U} & 23.2 & { }^{233} \mathrm{U} & 0.42 & & & \\ 238 \mathrm{Pu} & 0.63 & { }^{238} \mathrm{Pu} & 0.32 & & & \\ 239 \mathrm{Pu} & 67.1 & { }^{239} \mathrm{Pu} & 90.88 & 239 \mathrm{Pu} & 90.0 & \\ 240 \mathrm{Pu} & 4.21 & 240 \mathrm{Pu} & 7.20 & { }^{240} \mathrm{Pu} & 5.63 & 22 \\ 241 \mathrm{Pu} & 0.29 & 241 \mathrm{Pu} & 0.85 & 241 \mathrm{Pu} & 0.42 & \\ { }^{241} \mathrm{Am} & 4.6 & 241 \mathrm{Am} & - & 241 \mathrm{Am} & 3.89 & \end{array}$

NTS

$\begin{array}{lcl}{ }^{233} \mathrm{U} & 1.22 & \text { None } \\ { }^{238} \mathrm{Pu} & 0.15 & \\ { }^{239} \mathrm{Pu} & 96.1 & \\ { }^{240} \mathrm{Pu} & 0.98 \\ { }^{241} \mathrm{Pu} & 0.09 \\ { }^{241} \mathrm{Am} & 0.22 \\ { }^{244} \mathrm{Cm} & 0.84 \\ { }^{237} \mathrm{~Np} & 0.05 & \end{array}$

None 
Table 3.6 (Cont.)

CH Stored Waste $\quad$ RH Stored Waste Buried

Site (Isotope) (wt \%) (Isotope) (wt \%) (Isotope) (wt \%) Ref.

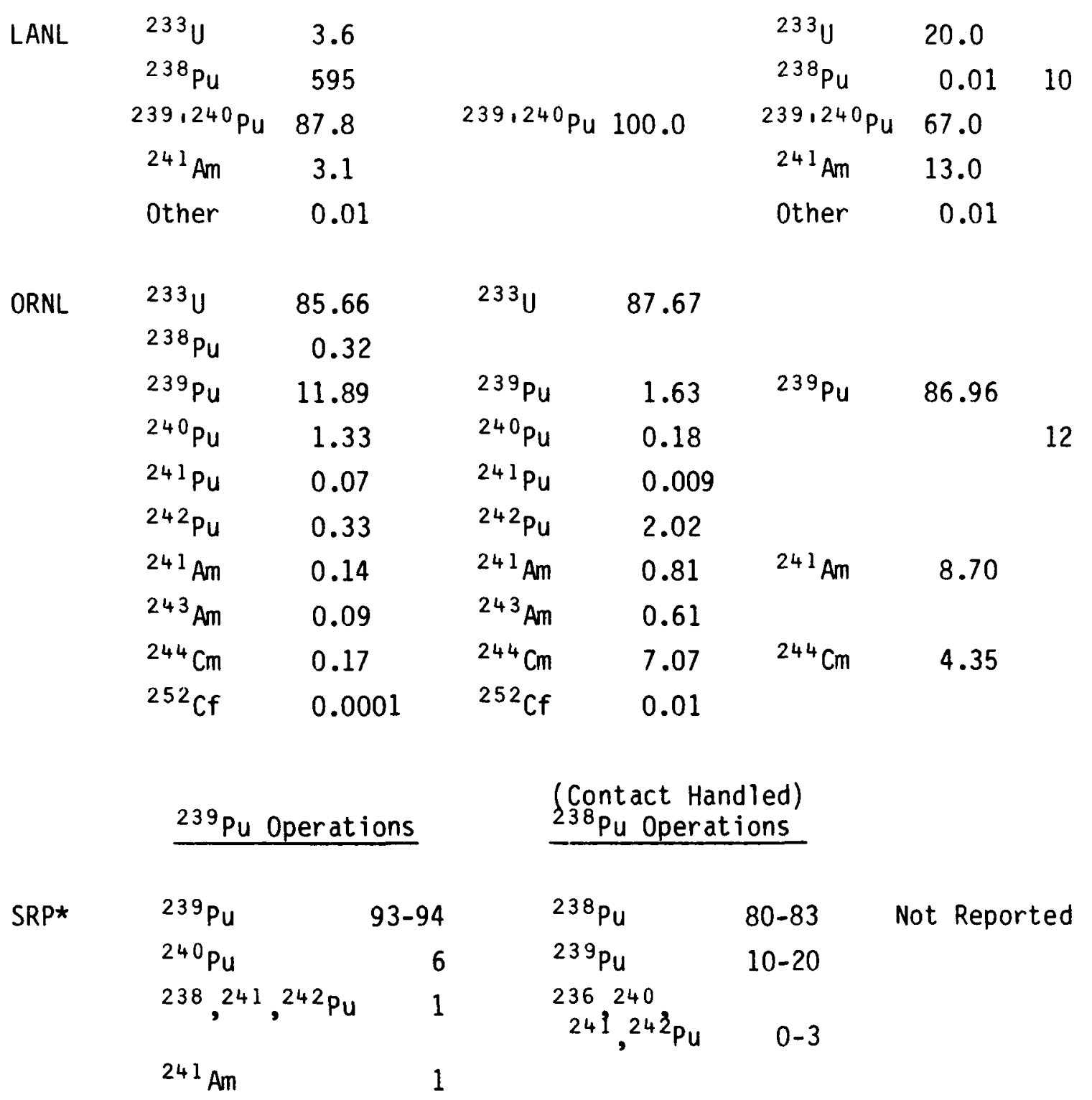

*Based on estimates from current waste streams. Stored wastes are similar, but not necessarily identical. At the present time $50 \%$ of waste is from ${ }^{238} \mathrm{Pu}$ operations and $50 \%$ is from ${ }^{239} \mathrm{Pu}$ operations. 
In his work in 1978, Shefelbine provides the best data available on how plutonium is distributed from package to package. ${ }^{15}$ Figures 3.7 and 3.8 are histograms that he obtained from the INEL. Figure 3.9 shows the probable content of drums at SRP. These data were also taken from the Shefelbine work. ${ }^{15}$

The maximum surface dose rate for contact-handled wastes is generally accepted to be $200 \mathrm{mR} / \mathrm{hr}$. Although unofficial, this is probably true for most contact-handled waste. Some wastes such as heat source curium waste at SRP are in containers incorporting heavy shielding to obtain those limits. Some containers at all of the sites could exceed $200 \mathrm{mR} / \mathrm{hr}$ if the shielding were not adequate. Concrete casks at ORNL and LANL are examples.

\subsection{Review of $\mathrm{CH}$ Waste Packages at Storage Sites}

Table 3.10 summarizes contact-handled waste categories at the storage sites. These data were tabulated from the detailed tables. Following the summary, Tables 3.11 through 3.46 present the details of each waste package at all sites. The information includes: (1) the total volume for each container type, (2) a description of the category, (3) how it is currently stored, (4) what the inside configuration is, and (5) as much detail as possible on the contents of each particular category. When not available, a best estimate of the contents is given. 


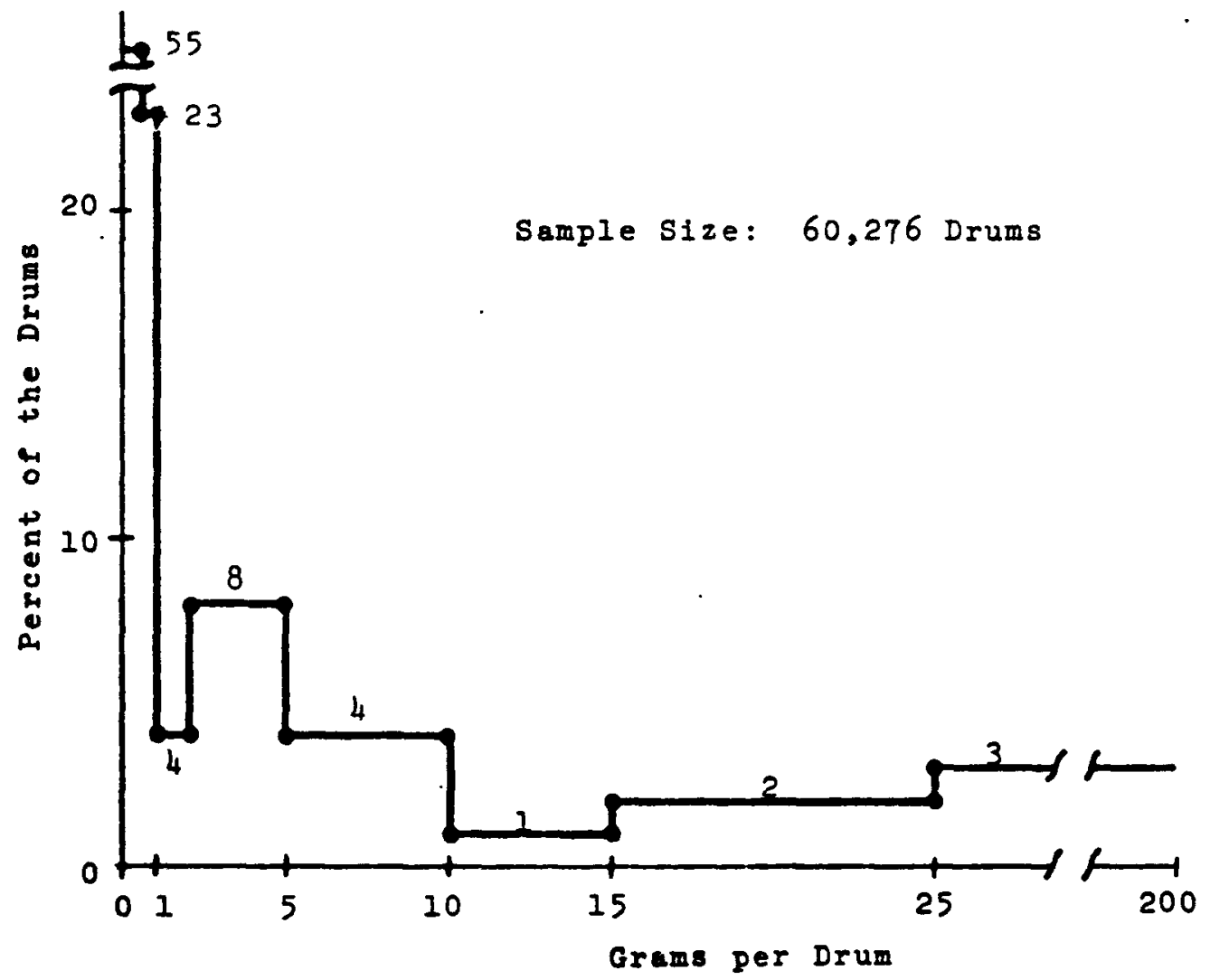

Figure 3.7 Histogram of $\mathrm{Pu}$ Content in Orums Stored at Idaho from $9-1-71$ to $12-31-77$

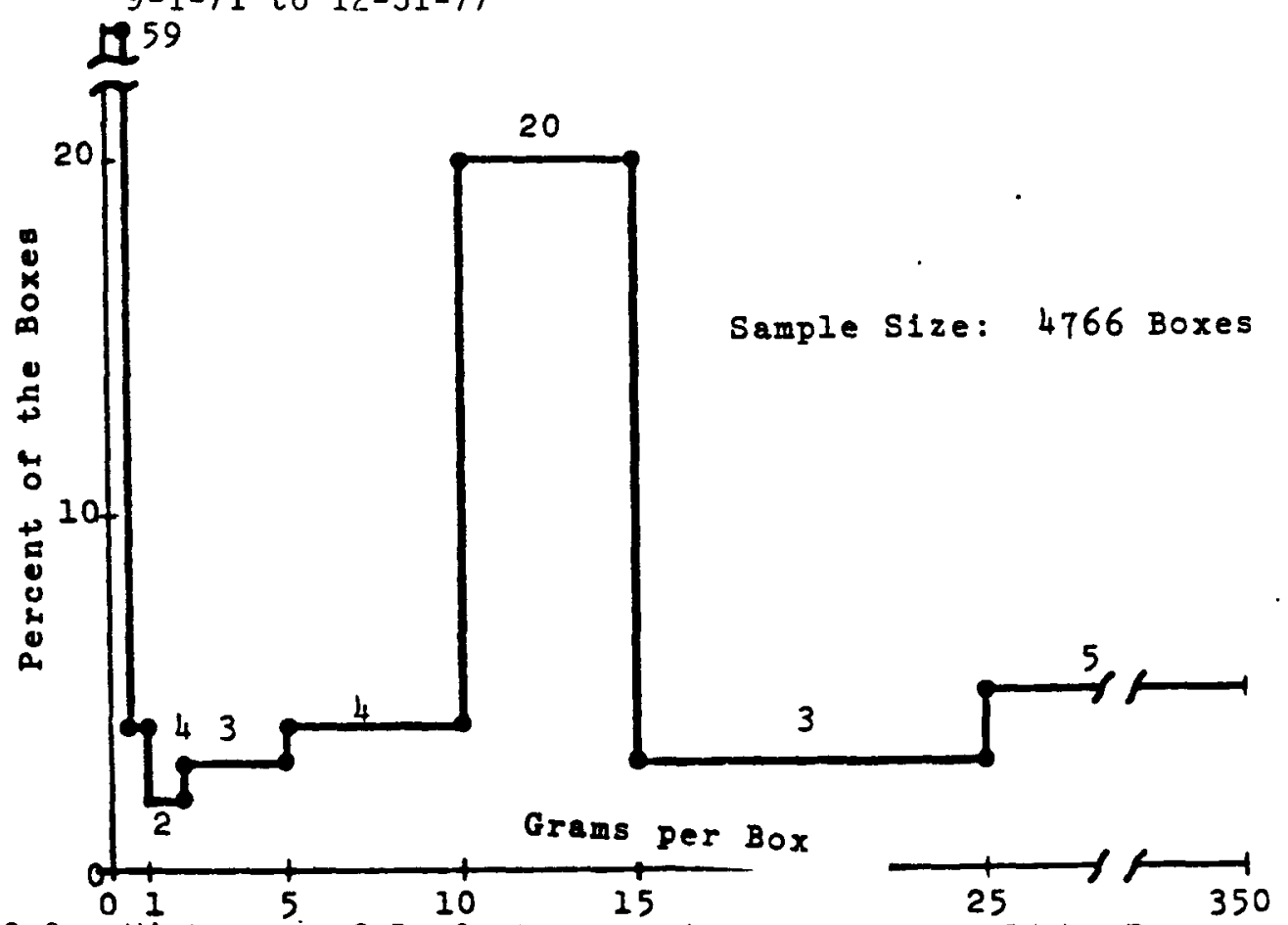

Figure 3.8 Histogram of Pu Content in Boxes stored at Idaho From 9-1-71 to 12-31-77 


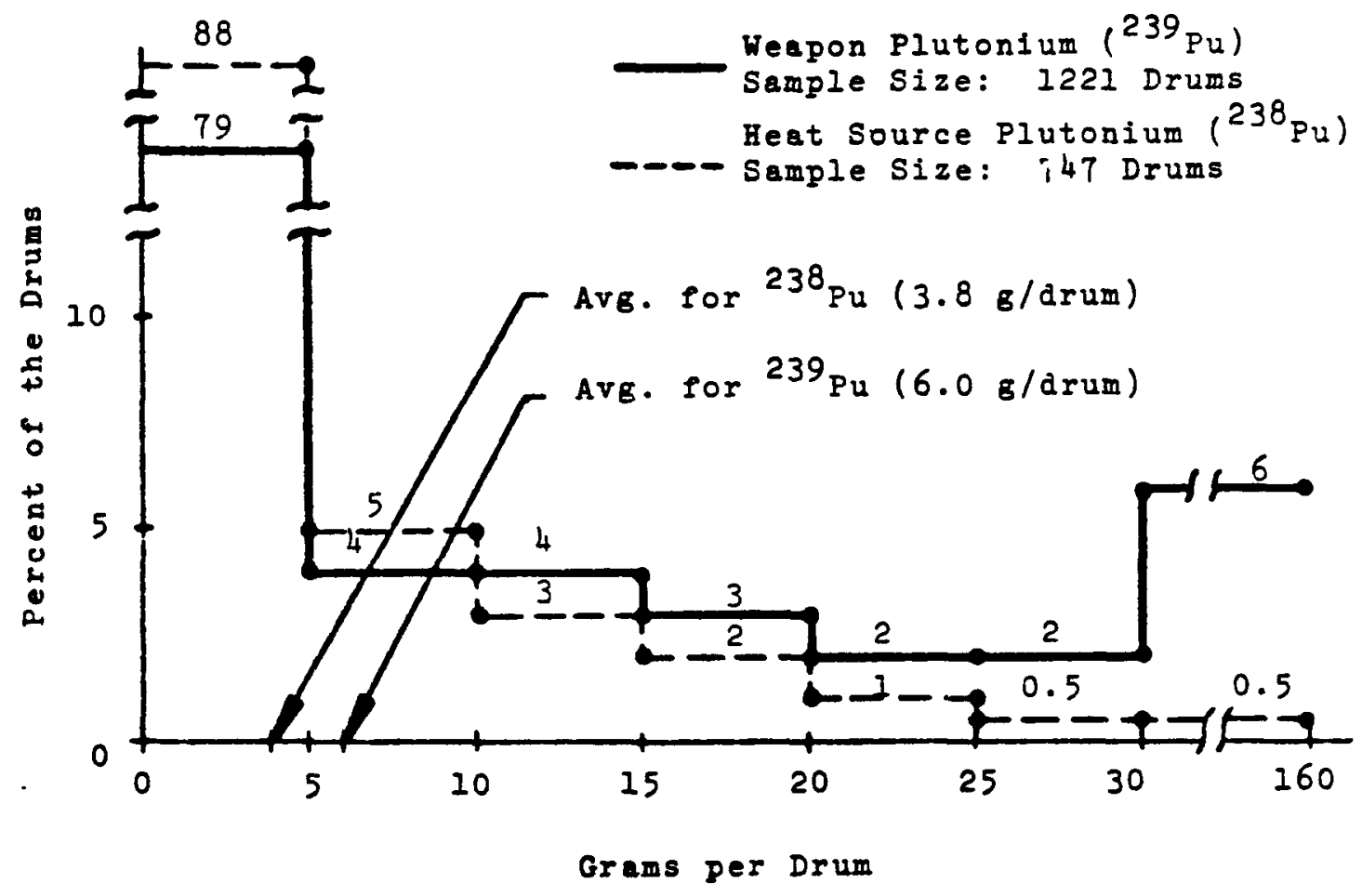

Figure 3.9 Histogram of Weapon and Heat Source Plutonium Content in 55-gallon Drums Produced by Savannah River from 7-1-74 to 6-31-77 
Table 3.10 Contact-Handled Packages at TRU Waste Storage Sites (To December 31,1980 )

\section{Contact-Handled Stored Waste}

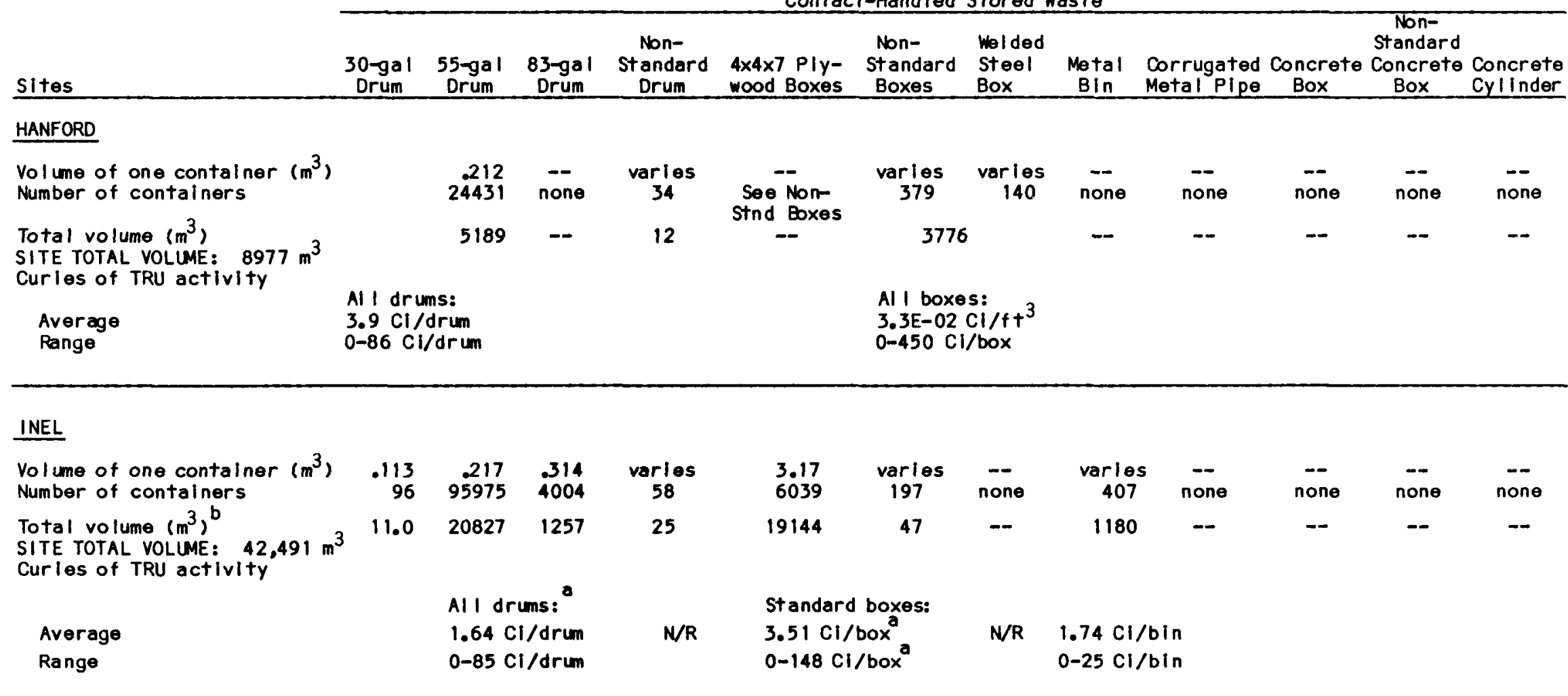

a Based on Rocky Flats drums and boxes only.

b The total contact handled volume reported here, $42,490.9 \mathrm{~m}^{3}$, varies from the Integrated Data Base total contact handled volume reported $42,352 \mathrm{~m}$, due to the façt that waste generators have used different contalner volumes. As an example, 4 'x4'x7' plywood boxes have been reported as $3.0 \mathrm{~m}^{3}$ and $112 \mathrm{ft}^{3}\left(3.17 \mathrm{~m}^{3}\right)$.

N/R Not Reported. 
Table 3.10 Contact-Handled Packages at TRU Waste Storage Sites (Continued) ( To December 31, 1980)

Contact-Handled Stored Waste

\begin{tabular}{|c|c|c|c|c|c|c|c|c|c|c|c|c|}
\hline \multirow[b]{2}{*}{ sites } & \multicolumn{12}{|c|}{ Contact-Handled Stored Waste } \\
\hline & $\begin{array}{c}30-g a l \\
\text { Drum }\end{array}$ & $\begin{array}{c}55-g a l \\
\text { Drum }\end{array}$ & $\begin{array}{c}83-g a 1 \\
\text { Drum }\end{array}$ & $\begin{array}{c}\text { Non- } \\
\text { St andard } \\
\text { Drum }\end{array}$ & $\begin{array}{l}4 \times 4 \times 7 \text { Ply- } \\
\text { wood Boxes }\end{array}$ & $\begin{array}{l}\text { Non- } \\
\text { Standard } \\
\text { Boxes }\end{array}$ & $\begin{array}{l}\text { Wolded } \\
\text { Steel } \\
\text { Box }\end{array}$ & $\begin{array}{c}\text { Metal } \\
\text { Bin } \\
\end{array}$ & $\begin{array}{l}\text { Corrugated } \\
\text { Metal Plpe }\end{array}$ & $\begin{array}{c}\text { Concrete } \\
\text { Box }\end{array}$ & $\begin{array}{c}\text { Non- } \\
\text { Standard } \\
\text { Concrete } \\
\text { Box } \\
\end{array}$ & $\begin{array}{l}\text { Concrete } \\
\text { Cylinder }\end{array}$ \\
\hline \multicolumn{13}{|l|}{ LANL } \\
\hline $\begin{array}{l}\text { Volume of one contalner }\left(\mathrm{m}^{3}\right) \\
\text { Number of contalners }\end{array}$ & $\begin{array}{r}.113 \\
514\end{array}$ & $\begin{array}{l}.217 \\
5216\end{array}$ & $\begin{array}{r}.314 \\
10\end{array}$ & $\begin{array}{c}\text { varies } \\
6\end{array}$ & \multirow[t]{2}{*}{$\begin{array}{l}\text { Incil. in } \\
\text { non-stnd }\end{array}$} & $\begin{array}{l}\text { varles } \\
308\end{array}$ & none & none & $\begin{array}{l}2.8 \\
211\end{array}$ & none & none & $\begin{array}{r}: 965 \\
306\end{array}$ \\
\hline $\begin{array}{l}\text { Total volume }\left(\mathrm{m}^{3}\right) \\
\text { SITE TOTAL VOLUME: } 4349 \mathrm{~m}^{3} \\
\text { Curies of TRU activity }\end{array}$ & 56 & 1060 & 3 & 10 & & 2334 & -- & -- & 591 & -- & -- & 295 \\
\hline $\begin{array}{l}\text { Average } \\
\text { Range }\end{array}$ & $\begin{array}{l}\mathrm{Cl} / \mathrm{drum} \\
\mathrm{cl} / \mathrm{drum}\end{array}$ & $\begin{array}{l}4.6 \mathrm{Cl} / \\
\mathrm{drum}\end{array}$ & $N / R$ & $N / R$ & \multicolumn{2}{|c|}{$\begin{array}{l}22.3 \mathrm{ci} / \mathrm{box} \\
0-150 \mathrm{ci} / \text { box }\end{array}$} & & & $N / R$ & & & $N / R$ \\
\hline
\end{tabular}

NTS

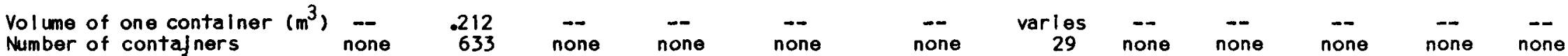
Total volume (m)

curies of TRU activity
Averago
$2.5 \mathrm{cl} / \mathrm{drum}$
$1.05 \mathrm{Ci} / \mathrm{box}$
Range
N/R
N/R

ORNL

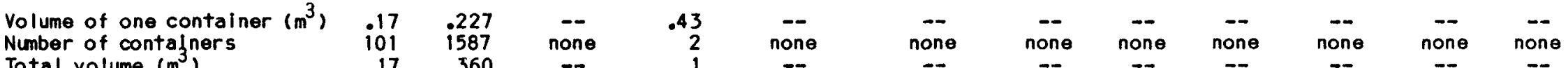

Total volume (m)

SITE TOTAL VOLLME: $378 \mathrm{~m}^{3}$

Curles of TRU activity

$17 \quad 360$

\begin{tabular}{ll} 
Average & Al I drums: \\
Range & $-7 \mathrm{Ci} / \mathrm{drum}$ \\
\hline & $\mathrm{N} / \mathrm{R}$
\end{tabular}

$N / R$

a Based on Rocky flats drums and boxes only.

N/R Not Reported 
Table 3.10 contact-Handled Packages at TRU Waste Storage Sites (Continued)

(To December 31, 1980)

Contact-Handled Stored Waste

\begin{tabular}{|c|c|c|c|c|c|c|c|c|c|c|c|c|}
\hline \multirow[b]{2}{*}{ Sites } & \\
\hline & $\begin{array}{c}\text { 30-gal } \\
\text { Drum }\end{array}$ & $\begin{array}{c}55-g a l \\
\text { Drum }\end{array}$ & $\begin{array}{c}83-g a l \\
\text { Drum }\end{array}$ & $\begin{array}{c}\text { Non- } \\
\text { Standard } \\
\text { Drum }\end{array}$ & $\begin{array}{l}4 \times 4 \times 7 \text { Ply- } \\
\text { wood Boxes }\end{array}$ & $\begin{array}{l}\text { Non- } \\
\text { Standard } \\
\text { Boxes }\end{array}$ & $\begin{array}{l}\text { Wolded } \\
\text { Steel } \\
\text { Box } \\
\end{array}$ & $\begin{array}{c}\text { Metal } \\
\text { Bin }\end{array}$ & $\begin{array}{l}\text { Corrugated } \\
\text { Metal Plpe }\end{array}$ & $\begin{array}{c}\text { Concrete } \\
\text { Box }\end{array}$ & $\begin{array}{l}\text { Non- } \\
\text { Standard } \\
\text { Concrete } \\
\text { Box }\end{array}$ & $\begin{array}{l}\text { Concrete } \\
\text { Cylinder }\end{array}$ \\
\hline \multicolumn{13}{|l|}{ SRP } \\
\hline Volume of one container $\left(\mathrm{m}^{3}\right)$ & $-\infty$ & .215 & - & $\rightarrow$ & -- & - & 11.3 & - & - & .99 & 18 & 7.6 \\
\hline Number of contalners & none & $7065^{c}$ & none & none & none & -- & 39 & none & none & 110 & 6 & 243 \\
\hline $\begin{array}{l}\text { Total vol une }\left(\mathrm{m}^{3}\right)^{\mathrm{d}} \\
\text { SITE TOTAL VOLLAME: } 3564 \mathrm{~m}^{3} \\
\text { Curles of TRU activity }\end{array}$ & -- & 1059 & - & -- & -- & - & 441 & -- & - & 109 & 108 & 1847 \\
\hline \multirow[t]{2}{*}{$\begin{array}{l}\text { Average } \\
\text { Range }\end{array}$} & & $\begin{array}{l}N / R \\
0-.5\end{array}$ & $\mathrm{Cl} / \mathrm{drum}$ & & & & $\begin{array}{l}N / R \\
N / R\end{array}$ & & & $\begin{array}{l}N / R \\
N / R\end{array}$ & $\begin{array}{l}N / R \\
N / R\end{array}$ & $\begin{array}{c}N / R \\
7-2000\end{array}$ \\
\hline & & & & & & & & & & & & $\mathrm{Cl} / \mathrm{cyln}$ \\
\hline TOTAL NUMBER OF CONTAINERS & 711 & 134,907 & 4014 & 100 & 6039 & 884 & 482 & 407 & 211 & 110 & 6 & 549 \\
\hline TOTAL VOLUME $\left(\mathrm{m}^{3}\right)$ & 83.6 & 28,629 & 1260 & 47.2 & 19144 & \multicolumn{2}{|c|}{6769.5} & 1180 & 590.8 & 109 & 108 & 2141.9 \\
\hline TOTAL VOLLME ALL SITES: 60,0 & $064 \mathrm{~m}^{3}$ & & & & & & & & & & & \\
\hline
\end{tabular}

a Based on Rocky flats drums and boxes only.

b The average radiation level is $205 \mathrm{mrem} / \mathrm{hr}$, thus these are considered contact handled. Some may exceed the $205 \mathrm{mrem} / \mathrm{hr}$.

The total number of drums here is the actual total number of 55 gal drums. Out of the 7065 drums shown in this column,

approximately 2141 are placed in concrete culverts. The volume on these drums is included in the concrete cylinder category.

d The total volume of $\mathrm{CH}$ waste that can be obtalned from these numbers does not agree with the Integrated Data Base number because

N/R Not reported.
Nolumes reported here are contalner volumes while the number supplied to the IDB is a waste volume. 
Table 3.11

\begin{tabular}{ccccc} 
Category & Number of Items & Total Volume & Ref. \\
\hline HANFORD & 55 -gallon drums & 24431 & $5189 \mathrm{~m}^{3}$ & 7,8 \\
\hline
\end{tabular}

\section{Description of Category:}

Cylindrical carbon steel, painted, or galvanized Dimensions: 22.5 in. ID $\times 33.25$ in. $L, 24$ in. $x 35$ in. $L,(.478$ in. wall) Volume $-7.42 \mathrm{ft}^{3}$

\# of carbon steel $=25150$

\# of galvanized $=1411$

\section{Placement:}

Below grade on asphalt pad. Drums are stacked $12 \mathrm{ft}$ high in modules. Plywood 5/8 in. thick separates layers. Covered with plywood and plastic and $4 \mathrm{ft}$. of dirt. Some drums are at $45^{\circ}$ angle in cement "V" trench.

\# in $\mathrm{V}$ trench $=1378$

\# on asphalt pad $=25183$

\section{Inside Configuration:}

Loose plastic double wrapped packages and cans. One hundred ninety of total have been mixed with cement. Majority of drums were lined with a plastic bag. Since 1980 a rigid polyethylene liner conforming to the shape of the drum has been used. Less than one drum in four compacted.

\section{Content Data:}

Specific contents unknown. Some segregation of combustible/noncombustible has occurred. Estimates are $-70 \%$ combustible, and $-30 \%$ noncombustible. TRU activity can only be estimated. Estimated $\mathrm{Pu}$ content $=1.226 \mathrm{~g} / \mathrm{ft}^{3}$-average, $27 \mathrm{~g} / \mathrm{ft}^{3}$-maximum. Estimated Radiation Level $=95 \%$ are 1 ess than $5 \mathrm{mR} / \mathrm{hr} ; 200 \mathrm{mR} / \mathrm{hr}$-maximum. 
Table 3.13

\begin{tabular}{lccc} 
Category & Number of Items & Total Volume \\
\hline HANFORD plywood boxes & 379 \\
\hline
\end{tabular}

\section{Description of Category:}

Fiberglass reinforced plywood boxes (FRP Boxes).

Dimensions vary: $4 \mathrm{ft} \times 4 \mathrm{ft} \times 7 \mathrm{ft}$ std. box, $12 \mathrm{ft} \times 10 \mathrm{ft} 6$ in. $\times 10 \mathrm{ft} 8 \mathrm{in} ., 16 \mathrm{ft} \times 9 \mathrm{ft} \times 10$ ft 8 in., $20 \mathrm{ft} \times 9 \mathrm{ft} \times 12 \mathrm{ft} 8$ in., $4 \times 4 \times 4,3 \times 3 \times 3,2 \times 2 \times 2$.

Volume: $8 \mathrm{ft}^{3}$ to $2300 \mathrm{ft}^{3}$

As seen above, dimensions can vary greatly. These are the most common sizes, but slight differences could exist from box to box.

\section{Placement:}

Placed below grade in earthen trenches, covered with plastic and $4 \mathrm{ft}$ of soil. Little or no

stacking. Larssen steel sheet pilings and railroad ties are used to shore up boxes.

\section{Inside Configuration:}

Loose waste in plastic wrap, large equipment is loose. Some have plastic liners, some taped. Not compacted.

\section{Content Data:}

Specific contents unknown. Mostly hoods, gloveboxes, or large items of equipment. Trash included in some, but well over $50 \%$ noncombustible. TRU activity estimated -

$\mathrm{Pu}$ content $=7.72 \times 10^{-2} \mathrm{~g} / \mathrm{ft}^{3}$-average, $1000 \mathrm{~g} /$ box-maximum.

Radiation level $=95 \%$ are less than $5 \mathrm{mR} / \mathrm{hr} ; 200 \mathrm{mR} / \mathrm{hr}$-maximum. 
Table 3.12

Category

Number of Items

Total Volume

Ref.

HANFORD non-standard drums $\quad 34 \quad 11.6 \mathrm{~m}^{3}$

\section{Description of Category:}

Cylindrical metal drums.

9-30 gal drums; volume $.116 \mathrm{~m}^{3} /$ drum

$25-110$ gal drums; volume $.425 \mathrm{~m}^{3} / \mathrm{drum}$

$110 \mathrm{gal}$ drums are 2-55 gal 16 gauge drums welded together

$110 \mathrm{gal}$ drums also referred to as $L-t$ in containers

\section{Placement:}

$110 \mathrm{gal}$ drums are stacked two high in the center of a module and then surrounded by other drums.

The floor of the module is asphalt. When the module is full, it is then covered with a $4 \mathrm{ft}$ deep dirt backfill.

\section{Inside Configuration:}

110 gal drum containers were constructed by filling with concrete and then boring a 5 in. diameter hole down the center. The cavity was then filled with a plastic pipe which could be capped with a plastic lid and sealed by a rubber gasket. The outer lid is held in place by a spring loaded clamp that is held in place with a bolt. The outer lid has a rubber gasket for sealing purposes.

\section{Content Data:}

$110 \mathrm{gal}$ drums were used to transport contaminated liquids. Even though the drums do not contain liquids, they have been buried. These drums have radiation levels averaging at $50 \mathrm{mR} / \mathrm{hr}$ and a maximum of $100 \mathrm{mR} / \mathrm{hr}$. 
Table 3.14

\begin{tabular}{cccc} 
Category & Number of Items & Total Volume & Ref. \\
\hline HANFORD steel boxes & 140 & 7,8 & 7 \\
\hline
\end{tabular}

\section{Description of Category:}

Carbon steel boxes, welded.

No special lugs or hooks.

Dimensions: vary $10 \mathrm{ft} 6$ in. $x 7 \mathrm{ft} 1-1 / 2 \mathrm{in.} \times 5 \mathrm{ft} 2-7 / 16 \mathrm{in.}, 16 \mathrm{ft} 6 \mathrm{in} . \times 7 \mathrm{ft} 1.5 \mathrm{in} . \times 5 \mathrm{ft}$ $2-1 / 2$ in., $13 \mathrm{ft} \times 5 \mathrm{ft} \times 10 \mathrm{ft}, 3 \times 3 \times 3$.

volume: $25 \mathrm{ft}^{3}$ to $650 \mathrm{ft}^{3}$

\section{Placement:}

Below grade in earthen trenches, covered with $4 \mathrm{ft}$ of soil. Limited stacking.

\section{Inside Configuration:}

Loose equipment. No liner. Lids are bolted in place. No compaction.

\section{Content Data:}

Specific contents unknown. Mostly hoods or equipment. TRU activity estimated$\mathrm{Pu}$ content $=7.72 \times 10^{-2} \mathrm{~g} / \mathrm{ft}^{3}$-average, $1000 \mathrm{~g} /$ box-maximum.

Radiation level $=$ All less than $5 \mathrm{mrem} / \mathrm{hr}$. 


\begin{tabular}{|c|c|}
\hline 55-gallon drums & 95975 \\
\hline
\end{tabular}

\section{Description of Category:}

DOT Specification $17 \mathrm{C}$ - Cylindrical steel drum, painted Dimensions: 22.5 in. ID $\times 33.25$ in. L, 24 in. $00 \times 35$ in. L (0.478 in. wall) Volume $-7.42 \mathrm{ft}^{3}$

Quantity received from Bettis APL have galvanized interior.

\section{Placement:}

Above grade on asphalt pads. Drums are stacked five drums high on $25 \mathrm{~m} \times 46 \mathrm{~m}$ cells or modules. They are in rectangular array, the sides being lined with fiberglass coated wooden boxes. Plywoor 1/2-in. thick separates the layers. The cell is covered with 5/8-in. fire retardant plywood, $20 \mathrm{mil}$ nylon polyvinyl sheeting, and $3 \mathrm{ft}$. of soil.

\section{Inside Configuration:}

Waste can be loose, in plastic bags, buckets, or smaller drums. After 1972 a polyvinyl liner was used. Approximately 42,280 drums contain liners, while 40,740 do not. Liner is 90-mil rigid polyvinyl, since 1978. Some waste is compacted, most is not. Sealed with rubber gasket and bolted closure ring. In addition, there are 17,113 drums which have been retrieved from the subsurface disposal area, placed in cargo containers or bins, and stored on the pads.

Content Data: See Table 3.16 which follows. 
Table 3.16 Content Data for 55-gal Drums at INEL*

\begin{tabular}{|c|c|c|c|c|c|c|}
\hline Content Codes & Content Description & $\begin{array}{l}\text { \# of } \\
\text { Drums }\end{array}$ & $\begin{array}{c}\text { Vol lume } \\
\mathrm{m}^{3}\end{array}$ & $\begin{array}{c}\text { Gross } \\
\text { Weight, } \\
\mathrm{Kg}\end{array}$ & $\begin{array}{l}\mathrm{Pu} \\
(\mathrm{g})\end{array}$ & $\begin{array}{l}\mathrm{Am} \\
(\mathrm{g})\end{array}$ \\
\hline
\end{tabular}

\section{INEL Designation}

$1,3,5,95,102,241$, $290,811,976,995$

Sludge -

Organic and inorganic process solids,12,360

liquid waste solidified with various sorbents, and dryed salts

$2,4,292,376,836$, 960

Concrete/Sludge - concrete filters or sludges that have been immobil-

ized with concrete

$10,153,202,330,336$, $337,460,463,801$, $802,804,847,900,970$

\begin{tabular}{|c|c|c|c|c|c|c|}
\hline $\begin{array}{l}20,480,481,803,825 \\
950,980\end{array}$ & $\begin{array}{l}\text { Metal - equipment, pipes, non- } \\
\text { compressible - noncombustible }\end{array}$ & 3,859 & 818 & 450,757 & 18,206 & 14 \\
\hline $335,338,490,805,813$ & Filters and Filter Media & 455 & 96 & 34,160 & 1,629 & 7 \\
\hline 374,990 & Dirt, Gravel, Asphalt & 1,439 & 300 & 295,455 & 375 & 0 \\
\hline 105,440 & Glass & 1,013 & 219 & 108,213 & 5,178 & 26 \\
\hline 0 & $\begin{array}{l}\text { Unknown - Waste buried prior to } \\
1970 \text {, exhumed and overpacked }\end{array}$ & 18,972 & 4,135 & $1,844,572$ & $24,958 * \star \star$ & $108 \star \star \star$ \\
\hline $300,301,310,311$ & $\begin{array}{l}\text { Graphite graphite cores, scarf- } \\
\text { ings, heels }\end{array}$ & 1,079 & 226 & 125,514 & 10,494 & 0 \\
\hline 441,442 & Raschig Rings - borosilicate glass & 2,268 & 473 & 247,054 & 13,571 & 9 \\
\hline
\end{tabular}

$2,776,075 \quad 35,108 \quad 13,607$

$13,909 \quad 2,949 \quad 3,321,902 \quad 28,173 \quad 206$

16,670

3,502

$1,435,869 \quad 8,570$ cloth, wood, gloves

Raschig Rings - borosilicate glass

2,643

206


Table 3.15 (Con't) Content Data for 55-gal Drums at INEL

\begin{tabular}{|c|c|c|c|c|c|c|}
\hline Content Codes & Content Description & $\begin{array}{l}\text { \# of } \\
\text { Drums }\end{array}$ & $\begin{array}{l}\text { Volume } \\
\mathrm{m}^{3}\end{array}$ & $\begin{array}{c}\text { Weiaht, } \\
\mathrm{Kg}\end{array}$ & $\begin{array}{l}P u \\
(q)\end{array}$ & $\begin{array}{l}A m \\
(\mathrm{~g})\end{array}$ \\
\hline 834 & Ac id & 859 & 179 & 167,496 & 91 & 0 \\
\hline \multirow[t]{2}{*}{835} & Caustic & 1,213 & 253 & 221,172 & 65 & 0 \\
\hline & Other & 5,094 & 845 & $\star \star$ & $\star \star$ & $\star \star$ \\
\hline \multicolumn{2}{|l|}{ Total } & 79,190 & 16,638 & $11,028,239$ & 146,415 & 14,355 \\
\hline \multicolumn{7}{|c|}{$\begin{array}{l}\text { * Data were taken from Transuranic Storage Area } 9-71 \text { through } 12-80 \text {. These data are found in the } \\
\text { Appendix and provide a much more detailed breakdown. No individual container data are available } \\
\text { for the first year of retrievable storage. } \\
\text { * Could not be calculated, see data in Appendix. }\end{array}$} \\
\hline
\end{tabular}


Table 3.17

\begin{tabular}{lcccc} 
& Category & Number of Items & Total Volume & Ref. \\
\hline INEL & 30 -gallon drums & 96 & $10.9 \mathrm{~m}^{3}$ & 20,22 \\
\hline
\end{tabular}

\section{Description of Category:}

Cylindrical steel drum, painted, 18 gauge. Dimensions: 19 in. ID $\times 29$ In. L

Volume: $4 \mathrm{ft}^{3}$

No longer accepted by INEL storage site.

\section{Placement:}

Stored above grade, on asphalt pads, similar to 55-gallon drums

\section{Inside Configuration:}

Waste is usually bagged in plastic, not compacted. Can be in cans or other containers, most have liners. Liner is 90-mil rigid polyvinyl, since 1978. Sealed with rubber gasket and bolted closure ring.

\section{Content Data:}

Specific contents are unknown. Most likely similar to 55-gallon drums with one exception. One-third of all 55-gallon drums contains sludge. No sludge is known to have been packed in 30-galion drums. 
Table 3.18

\begin{tabular}{lllll} 
& Category & Number of Items & Total Volume & Ref . \\
\hline INEL & $83-g$ allon drums & 4004 & $1257 \mathrm{~m}^{3}$ & 20,22 \\
\hline
\end{tabular}

\section{Description of Category:}

Cylindrical steel drum, painted.

Dimensions: 26 in. ID $\times 43$ in. L

Volume: $11.1 \mathrm{ft}^{3}$

No longer accepted by INEL storage site. Used at INEL for overpacking damaged containers, primarily those from SDA.

These are not DOT-approved containers.

\section{Placement:}

\section{Inside Configuration:}

Waste can be in cans, boxes, or bagged in plastic. Since 1972, drums have liners of polyvinyl plastic, but specifications unknown. Not compacted. Sealed with rubber gasket and holted closure ring.

\section{Content Data:}

Specific contents are unknown. Most likely similar to contents in 55 gallon drums. The 83-gallon drum is also used to overpack damaged or retrieved 55-gailion drums. 
Table 3.19

\begin{tabular}{lllll} 
Category & Number of Items & Total Volume & Ref. \\
\hline INEL & $\begin{array}{l}\text { Drums in Cargo } \\
\text { Containers and Bins }\end{array}$ & 489 & $3596 \mathrm{~m}^{3}$ (drums only) & 20,22 \\
\hline
\end{tabular}

\section{Description of Category:}

17,113 fifty-five gallon drums have been overpacked in 209 cargo carriers and 280 bins. See description of bins in Table 3.24

Dimensions: (Cargo Carrier) Rectangular, approximately $8 \mathrm{ft} \times 8 \mathrm{ft} \times 20 \mathrm{ft}$.

Volume: Drum - $7.42 \mathrm{ft}^{3}$, cargo carrier $-1280 \mathrm{ft}^{3}$

\section{Placement:}

Cargo container placed above grade on asphalt pads. Drums are placed inside cargo container. Container holds approximately 72 drums. Bins are sometimes used to surround modules of 55 gallon drums.

\section{Inside Configuration:}

These 55-gallon drums are from the retrieval operation which exhumed drums buried in 1970 . Drums were sealed with a rubber gasket and bolted metal closure ring. Drums were placed in plastic bags prior to overpacking in cargo containers, bins, or 83-gallon drums.

\section{Content Data:}

Typical buried waste of which there are no specific records. Mix of waste is probably similar to 55-gallon stored drums, except some soil is present from the retrieval operation. 
Table 3.20

\begin{tabular}{lcccc} 
& Category & Number of Items & Total Volume & Ref. \\
\hline INEL & Non-Standard Drums & 58 & $25 \mathrm{~m}^{3}$ & 20,22 \\
\hline
\end{tabular}

\section{Description of Category:}

Various size cylindrical metal drums.

Volume: $1-1 \mathrm{ft}^{3}, 1-5 \mathrm{gallon}, 1-50 \mathrm{ft}^{3}$

$1-3 \mathrm{ft}^{3}, 1-200$ gallon, 53 - 110 gallon (double 55 gallon)

Double 55 gallon containers are two 55-gallon drums welded together.

\section{Placement:}

Smaller containers may be inside 55-gallon drum. Placed on asphalt pad similar to 55 gallon drums.

\section{Inside Configuration:}

Waste is in boxes, smaller cans, or bagged in plastic, not compacted. Most do not have liners. Sealed with rubber gasket and bolted closure ring.

\section{Content Data:}

Specific contents unknown. Most likely similar to 55-gallon drums except for sludges which are in $55-g$ allion drums. 
Table 3.21

\begin{tabular}{|c|c|c|c|c|}
\hline & Category & Number of Items & Total Volume & Ref. \\
\hline INEL & $\begin{array}{l}\text { Plywood Boxes } \\
\text { (Standard) }\end{array}$ & 6039 & $19144 \mathrm{~m}^{3}$ & 20,22 \\
\hline
\end{tabular}

\section{Description of Category:}

Plywood box 3/4-in. thick. Dimensions: $4 \mathrm{ft} \times 4 \mathrm{ft} \times 7 \mathrm{ft} . *$ Volume: $112 \mathrm{ft}^{3}$

Since 1972 boxes covered with layer of fiberglass. Thickness of fiberqlass and box may vary. Usually $3 / 4$ in. wood, $1 / 8$ in. nominal thickness fiberglass

\# of plain boxes $-1262 \simeq 4000 \mathrm{~m}^{3}$

\# of fiberglass boxes - $4974 \simeq 15780 \mathrm{~m}^{3}$

* Actual dimensions may vary up to 5 in. greater in each dimension.

\section{Placement:}

of Above grade on asphalt pads. Ised to surround the cells of 55-gallon drums. Contained in cells $25 \mathrm{~m} \times 46 \mathrm{~m}$. Three layers are separated by $1 / 2 \mathrm{in}$. plywood, covered with $5 / 8$ in. plywood, $20 \mathrm{mil}$ nylon polyvinyl sheeting, and $3 \mathrm{ft}$ of soil.

\section{Inside Configuration:}

Equipment or loose waste is enclosed in plastic or plastic bags. May contain drums or other packages of loose waste. Boxes contain a fiberboard liner and poly box.

\section{Content Data:}

See Table 3.22 which follows. 


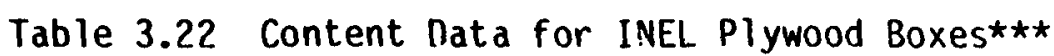

\begin{tabular}{|c|c|c|c|c|c|}
\hline Waste Codes & Description & $\begin{array}{c}\text { Volume } \\
\mathrm{m}^{3}\end{array}$ & $\begin{array}{l}\text { Weight, } \\
\mathrm{Ka}\end{array}$ & $\begin{array}{l}\mathrm{Pu} \\
(\mathrm{q})\end{array}$ & $\begin{array}{l}\text { Am } \\
(q)\end{array}$ \\
\hline $5,976,978,995$ & $\begin{array}{l}\text { Sludge - Organic and inorganic process } \\
\text { solids, liquid, waste solidified with } \\
\text { various sorbents, and dried salts. }\end{array}$ & 127 & 80,360 & 85 & 2 \\
\hline $\begin{array}{l}330,336,337 \\
900,970\end{array}$ & $\begin{array}{l}\text { Combust ibles - rags, paper, plastic, } \\
\text { cloth, wood }\end{array}$ & 3,386 & 919,178 & 9,594 & 757 \\
\hline 338,490 & Filters or Filter Media & 2,325 & 631,060 & $13,86.3$ & 17 \\
\hline 374,842 & Dirt, gravel, asphalt & 253 & 139,890 & 273 & 0 \\
\hline $480,481,824,950$ & Metals & 10,902 & $4,419,440$ & 32,757 & 58 \\
\hline 376,960 & $\begin{array}{l}\text { Concrete cemented sludge, cemented } \\
\text { filters }\end{array}$ & 314 & 330,4165 & 2,178 & 11 \\
\hline \multirow[t]{2}{*}{302} & Benelex and plexiglass & 50 & 26,700 & 63 & 0 \\
\hline & Other* & 2,373 & $\star \star$ & $\star \star$ & $\star \star$ \\
\hline TOTAL & & 19,780 & $6,547,044$ & 58,913 & 855 \\
\hline
\end{tabular}

* Includes waste not categorized

* Could not be calculated, see data in Appendix.

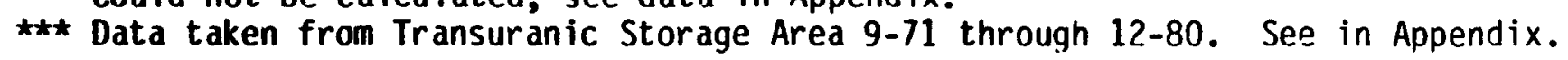




\section{Table 3.23}

Category

Number of Items

Total Volume

Ref.

INEL Non-St andard

197

$47 \mathrm{~m}^{3}$

20,22

Plywood Boxes

Description of Category:

Plywood boxes of various sizes.

Volumes:

$1-25 \mathrm{ft}^{3}, 5-72 \mathrm{ft}^{3}, 1-121 \mathrm{ft}^{3}, 2-155 \mathrm{ft}^{3}, 4-378 \mathrm{ft}^{3}$,

$1-46 \mathrm{ft}^{3}, 9-84 \mathrm{ft}^{3}, 4-130 \mathrm{ft}^{3}, 6-169 \mathrm{ft}^{3}, 3^{2}-424 \mathrm{ft}^{3}$,

$142-56 \mathrm{ft}^{3}, 8-93 \mathrm{ft}^{3}, 7-144 \mathrm{ft}^{3}, 3-188 \mathrm{ft}^{3}$.

\section{Placement:}

Above ground on asphalt pads. Same as standard size boxes.

\section{Inside Configuration:}

Same as standard size boxes. Waste is usually bagged in plastic or contained in other drums or boxes.

\section{Content Data:}

Specifics on each box are unknown. Most larger than standard size contain large metal equipment or gloveboxes. Smaller sizes most likely equipment, but could be similar to mix in standard size boxes. 
Table 3.24

\begin{tabular}{lcccc} 
& Category & Number of Items & Total Volume & Ref. \\
\hline INEL & Metal Bin & $407 *$ & $1180 \mathrm{~m}^{3}$ & 20,22 \\
\hline
\end{tabular}

\section{Description of Category:}

Sheet metal steel bins. Painted with white paint. 12 gauge steel.

Dimensions: $723 / 8$ in. high, $503 / 8$ in. long, $583 / 8$ in. deep.

Volume: $120 \mathrm{ft}^{3}$

Stiffner: 3 in. angle iron across bottom

Braces: 2 in. angle iron in corners at top, diagonally.

* Category does not include 280 bins used to overpack 55-gallon drums containinq retrieval of buried waste. (See Table 3.19).

\section{Placement:}

Above ground on asphalt pads. Same as standard size plywood boxes.

\section{Inside Configuration:}

Sealed with rubber gasket. Weather stripping $7 / 16$ in. $x 1$ in. wide. Cover held on with $5 / 16$ in. hex head screw.

72 bins contain waste that was retrieved from earlier buried TRU waste. This is the same type of waste as the 280 bins above, but is not to be confused as part of it.

\section{Content Data:}

See Table 3.25 on following page. 
Table 3.25 Content of INEL Metal Bins*

\begin{tabular}{|c|c|c|c|c|c|}
\hline Waste Codes & Description & $\begin{array}{l}\text { No. of } \\
\text { Bins }\end{array}$ & $\begin{array}{c}\text { Volume } \\
\mathrm{m}^{3}\end{array}$ & $\begin{array}{l}\text { Weiqht, } \\
\text { Kq }\end{array}$ & $\begin{array}{l}\mathrm{Pu} \\
(\mathrm{q})\end{array}$ \\
\hline 0 & Not Recorded - Unknown & 72 & 206 & 67,252 & 948 \\
\hline 100 & $\begin{array}{l}\text { Laboratory Waste } 50 \% \text { to } 70 \% \\
\text { combustible, small equipment, } \\
\text { filters, cans }\end{array}$ & 261 & 746 & 252,742 & 415 \\
\hline 101 & Cut-up Glove Boxes & 44 & 126 & 48,444 & 250 \\
\hline 201 & Noncombustible Solids & 27 & 77 & 32,314 & 69 \\
\hline 202 & $\begin{array}{l}\text { Combustibles - paper, cloth, } \\
\text { plastic, etc. }\end{array}$ & 5 & 14 & 3,488 & $1 n$ \\
\hline 203 & $\begin{array}{l}\text { Miscellaneous - paper, cloth, } \\
\text { metal, glass }\end{array}$ & 4 & 11 & 2,858 & 0 \\
\hline TOTAL & & 413 & 1,180 & 403,610 & 1,692 \\
\hline
\end{tabular}

* Data taken from Transuranic Storage Area 9-71 through 12-80. Found in Appendix. 
Table 3.26

\begin{tabular}{lcccc} 
& Category & Number of Items & Total Volume & Ref. \\
\hline LANL & 30 -gallon drums & 514 & $55.5 \mathrm{~m}^{3}$ & 9,10 \\
\hline
\end{tabular}

\section{Description of Category:}

Cylindrical $17 \mathrm{H}$ mild steel (black iron) drum. Dimensions: $0.48 \mathrm{~m}$ ID $\times 0.74 \mathrm{~m} \mathrm{~L}$

Volume: $3.81 \mathrm{ft}^{3}$

Drums used only through $\sim 1975$.

\section{Placement:}

Stacked on below ground storage pad.

\section{Inside Configuration:}

Loose waste administratively segregated within TRU work areas. Glovebox waste packaged in small cans, boxes, or bagged in plastic. Sealed with rubber gasket.

\section{Content Data:}

See Table 3.27 which follows. Also see LANL data in Appendix. Major Radionuclides: Pu-239.

$0-0.100 \mathrm{R} / \mathrm{hr}$ gamma at contact. 
Table 3.27 Content of LANL 30-Gallon Drums*

\begin{tabular}{|c|c|c|c|c|c|c|}
\hline Content Codes & Content Description & $\begin{array}{l}\text { Volume } \\
\mathrm{m}^{3}\end{array}$ & $\begin{array}{c}\text { Weight, } \\
\mathrm{Kg}\end{array}$ & $\begin{array}{l}\text { No. } \\
\text { Drums }\end{array}$ & $\begin{array}{c}\text { Pu-239 } \\
(q)\end{array}$ & $\begin{array}{c}\text { Pu-238 } \\
\text { (q) }\end{array}$ \\
\hline$A 15, A 18, A 60$ & $\begin{array}{l}\text { Combustibles - mixed cellulosics, } \\
\text { mixed paper, plastic, rubber, } \\
\text { other }\end{array}$ & 13.7 & 4215.3 & 135 & 1,236 & 1 \\
\hline A16 & Combustibles - plastic material & 1.0 & 244.8 & 9 & 21.6 & 0 \\
\hline A19 & $\begin{array}{l}\text { Mixed combustibles - noncom- } \\
\text { bustible }\end{array}$ & 4.3 & 1,068 & 38 & 82.8 & 0 \\
\hline$A 25, A 70$ & $\begin{array}{l}\text { Sludges - leaded process residue, } \\
\text { chemical waste }\end{array}$ & 26.2 & 11169.3 & 235 & 6781.9 & .3 \\
\hline$A 30, A 31, A 50$ & $\begin{array}{l}\text { Metal - equipment, metal } \\
\text { crucibles, scrap, dies, precious } \\
\text { metals, other scrap metal }\end{array}$ & 5.7 & 2732.8 & 57 & 274.6 & 6.8 \\
\hline $\begin{array}{l}\text { A21, A46, A55, } \\
\text { A61, A95, A99 }\end{array}$ & Other & 4.5 & 1617.3 & 40 & 44.9 & $\underline{0}$ \\
\hline TOTAL & & 55.4 & 21047.5 & 514 & 7229.4 & 8.1 \\
\hline
\end{tabular}

* Data taken from LANL Stored TRU Waste Data, found in Appendix. 
Table 3.28

\begin{tabular}{|c|c|c|c|c|}
\hline & Category & Number of Items & Total Volume & Ref. \\
\hline LANL & 55-gallon drums & 5216 & $1060.2 \mathrm{~m}^{3}$ & 9,10 \\
\hline
\end{tabular}

\section{Description of Category:}

Cylindrical $17 \mathrm{C}$ mild steel (black iron) drum.*

Dimensions: $0.89 \mathrm{~m}$ ID $\times 0.61 \mathrm{~m}$ ID

Volume: $7.18 \mathrm{ft}^{3}$

Since 1975 obtained from Rocky Flats to RFP specifications.

* Note: The outside of all LANL drums has been covered with a corrosion resistant grease. Should the drums require shipment, the grease could be removed.

\section{Placement:}

Stacked in pad-type storage (above and below ground).

\section{Inside Configuration:}

Loose waste administratively separated in TRU work areas. Glovebox waste packaged in small cans, boxes, or bagged in plastic. Prior to 1975 sealed with rubber gasket. Since 1975 Rocky Flats specifications used. Liners are used as deemed necessary. All sludge drums, soil, machine oils, and certain special cases have utilized a liner.

\section{Content Data:}

See Table 3.29 which follows. Also see LANL data in Appendix. Major Radionuclides: Pu-239, Pu-238, AM-241, and Mixed Fission Products. $0-0.300 \mathrm{R} / \mathrm{hr}$ gamina at contact. 
Table 3.29 Content of LANL 55-Gallon Drums*

\begin{tabular}{|c|c|c|c|c|c|c|c|c|}
\hline Content Codes & Content Description & $\begin{array}{c}\text { Volume } \\
\mathrm{m}^{3}\end{array}$ & $\begin{array}{l}\text { Weight, } \\
\mathrm{Kg}\end{array}$ & $\begin{array}{l}\text { No. } \\
\text { Drums }\end{array}$ & $\begin{array}{l}\text { Pu-239 } \\
(\mathrm{g})\end{array}$ & $\begin{array}{l}P u-238 \\
(\mathrm{~g})\end{array}$ & $\begin{array}{l}A m-241 \\
(a)\end{array}$ & $\begin{array}{l}0.2 .33 \\
(\mathrm{~g})\end{array}$ \\
\hline$A 15, A 18, A 60$ & $\begin{array}{l}\text { Combustibles - mixed } \\
\text { cellulosics, mixed paper, } \\
\text { plastic, rubber, other } \\
\text { combustibles }\end{array}$ & 335.9 & 93668.1 & 1,672 & 13920.2 & 42.3 & 2.1 & 0 \\
\hline A16 & Plastic material & 3.8 & 1,017 & 19 & 81.2 & 0 & 0 & n \\
\hline A19 & $\begin{array}{l}\text { Mixed combustibles - } \\
\text { noncombustible }\end{array}$ & 15.6 & 4,068 & 75 & 134.8 & $<0.1$ & 0 & 0 \\
\hline$A 25, A 70, A 75$ & $\begin{array}{l}\text { Sludges - leached process, } \\
\text { residue, chemical waste } \\
\text { chemical treatment sludge }\end{array}$ & 306 & 264620.1 & 1,480 & 22773.8 & 6.4 & 296.6 & 0 \\
\hline A76 & $\begin{array}{l}\text { Cemented sludge - cement } \\
\text { paste }\end{array}$ & 74.1 & 88,150 & 357 & 69.1 & .2 & 6.5 & 2.1 \\
\hline $\begin{array}{l}A 30, A 31, A 50, \\
A 52\end{array}$ & $\begin{array}{l}\text { Metal - equipment, metal } \\
\text { crucibles, scrap, dies, } \\
\text { other scrap metals }\end{array}$ & 71 & 27146.6 & 355 & 1976.2 & 44.1 & 0 & 0 \\
\hline A55, A56 & Filters and Filter Media & 2.3 & 1589.9 & 11 & 5.9 & $<0.1$ & 0 & 0 \\
\hline A90 & So il & 9.6 & 8,610 & 46 & 0.6 & 0 & 0 & 0 \\
\hline $\begin{array}{l}A 10, A 20, A 21, \\
A 61, A 95, A 99\end{array}$ & Other & 241.9 & 85482.6 & $\underline{1,201}$. & 9649.4 & 69.9 & 13.4 & 1 \\
\hline TOTAL & & 1060.2 & $574,352.2$ & 5,216 & 48611.2 & 162.9 & 313.6 & 3.1 \\
\hline
\end{tabular}

* Data taken from LANL Stored TRU Waste Data, found in Appendix. 
Table 3.30

\begin{tabular}{llccc} 
& Category & Number of Items & Total Volume & Ref \\
\hline LANL & Corrugated Metal Pipe & 211 & $590.8 \mathrm{~m}^{3}$ & \\
\hline
\end{tabular}

\section{Description of Category:}

Corrugated metal pipe

Dimensions: $20 \mathrm{ft} \times 2.5 \mathrm{ft}$

Placement:

Pipes stacked vertically in shallow pit, pit then backfilled with dirt.

Inside Configuration:

Construction consisted of taking the corruqated pipe and welding a metal plate to one end and then filling with $1 \mathrm{ft}$ of clean concrete. The waste which was not dewatered was then added. Concrete was added to the slurry to form a cement paste. After this hardened, another $1 \mathrm{ft}$ of concrete was then added to the top of the pipe. No metal plate or lid was placed in the top of the pipe. Fach pipe is identified with an I.D. tag.

\section{Content Data:}

Contents are PU-239/Am-241 in a cement paste. Radiation levels are less than $200 \mathrm{mR} / \mathrm{hr}$. 
Table 3.31

\begin{tabular}{|c|c|c|c|c|}
\hline & Category & Number of Items & Total Volume & Ref. \\
\hline LANL & 83-gallon drums & 10 & $3.14 \mathrm{~m}^{3}$ & 9,10 \\
\hline
\end{tabular}

\section{Description of Category:}

Cylindrical mild steel (black iron) drum, 15 gauge body and heads. Dimensions: $0.70 \mathrm{~m} \mathrm{ID} \times 0.95 \mathrm{~m} \mathrm{~L}$.

Volume: $0.314 \mathrm{~m}^{3}$

Used only to overpack damaged 55 gallon drums.

\section{Placement:}

Stacked in pad-type storage (above and below ground).

\section{Inside Configuration:}

Overpack for damaged 55-gallon drums, sealed with two rolled or swedged-type hoops, 12 gage bolted ring with drop forged lugs and $5 / 8$ in. bolt.

\section{Content Data:}

To date, contains on ly sludge and cement paste.

Major Radionuclides: Pu-239, Am-241

$0-0.003 \mathrm{R} / \mathrm{hr}$ gamma at contact. 
Table 3.32

\begin{tabular}{lcccc} 
& Category & Number of Items & Total Volume & Ref. \\
\hline LANL Non-Standard Drums & 6 & $9.9 \mathrm{~m}^{3}$ & 9,10 \\
\hline
\end{tabular}

\section{Description of Category:}

Oversized mild steel drums with removable heads.

Dimensions: 4 drums measuring $0.92 \mathrm{~m}$ diameter ID $\times 1.82 \mathrm{~m} \mathrm{~L}$

Volume: 4 drums measuring $1.21 \mathrm{~m}$ diameter $\times 1.82 \mathrm{~m} \mathrm{~L}$

Drums thought to be military surplus.

\section{Placement:}

Placed on below ground storage pad.

\section{Inside Configuration:}

Sealed with rubber gasket. Wastes are from laboratory decontamination following accidental release. Plastic bags and wrapped items fill drums.

\section{Content Data:}

Equipment and other debris.

Major Radionuclides: Pu-239

0.0 R/hr at contact. 
Table 3.33

\begin{tabular}{|c|c|c|c|c|}
\hline & Category & Number of Items & Total Volume & Ref. \\
\hline LANL & Plywood Boxes & 308 & $2334.1 \mathrm{~m}^{3}$ & 9,10 \\
\hline
\end{tabular}

\section{Description of Category:}

Fiberglass reinforced plywood box.

Dimensions: $1.2 \times 1.2 \times 2.1 \mathrm{~m}$ or made to order for special sizes up to $2.4 \times 3 \times 10.6 \mathrm{~m}$

Volume: Standard $-3.17 \mathrm{~m}^{3}$ Rocky Flats Plant specification. (see INEL boxes).

\# Standard size - 100

\# Larger than standard - 159

\# Smaller than standard - 49

\section{Placement:}

Stacked on pad-type storage, above or below ground.

\section{Inside Configuration:}

Waste items may be wrapped in plastic, painted, or have openings sealed to contain contamination.

\section{Content Data:}

See Table 3.34 which follows. Also see Appendix, LANL section.

Major Radionuclides: Pu-239, Pu-238, Mixed Fission Products

$0-0.070 \mathrm{R} / \mathrm{hr}$ gamma at contact with one exception, reading $1.5 \mathrm{R} / \mathrm{hr} \max$. 
Table 3.34 Content of LANL FRP Boxes*

\begin{tabular}{|c|c|c|c|c|c|c|c|}
\hline Content Codes & Content Description & $\begin{array}{c}\begin{array}{c}\text { Volume } \\
\mathrm{m}^{3}\end{array} \\
\end{array}$ & $\begin{array}{c}\text { Weight, } \\
\mathrm{Kg}\end{array}$ & $\begin{array}{c}\text { No. } \\
\text { Boxes }\end{array}$ & $\begin{array}{c}\text { Pu-239 } \\
(\mathrm{g})\end{array}$ & $\begin{array}{c}\text { Pu-238 } \\
(\mathrm{g})\end{array}$ & $\begin{array}{c}A m-24 I \\
(\mathrm{~g})\end{array}$ \\
\hline A19 & $\begin{array}{l}\text { Mixed combustibles - } \\
\text { noncombustible }\end{array}$ & 13.6 & 881.9 & 4 & 2001.3 & 0 & 0 \\
\hline $\mathrm{A} 30, \mathrm{~A} 31, \mathrm{~A} 52$ & $\begin{array}{l}\text { Metal - equipment, scrap } \\
\text { metal }\end{array}$ & 2096.9 & $201,17.2$ & 234 & 8085.7 & 160 & .3 \\
\hline A35 & $\begin{array}{l}\text { Combustible - Combustible } \\
\text { building debris }\end{array}$ & 7.3 & 51.7 & 2 & $8.8-$ & 0 & 0 \\
\hline A55 & Filters - Filter Media & 117.4 & 7857.0 & 35 & 1.1 & $<.1$ & 0 \\
\hline A90 & Soil & 69.4 & 42910.5 & 27 & 98.3 & 0 & 0 \\
\hline A36, A85, A99, & Other & 29.5 & 2912.9 & 6 & 450.4 & $\underline{0}$ & 0 \\
\hline TOTAL & & 2334.1 & $255,791.2$ & 308 & 10645.6 & 160 & .3 \\
\hline
\end{tabular}


Table 3.35

\begin{tabular}{|c|c|c|c|c|}
\hline & Category & Number of Items & Total Volume & Ref. \\
\hline LANL & Concrete Cask & 306 & $294.9 \mathrm{~m}^{3}$ & 9,10 \\
\hline
\end{tabular}

\section{Description of Category:}

Concrete Casks containing two 30 gallon drums.

Dimensions: $0.90 \mathrm{~m} \mathrm{OD}(\max ) \times 1.82 \mathrm{~m} \mathrm{~L}$

Volume: $0.965 \mathrm{~m}^{3}$

Casks constructed in accordance with ASTM C76 specifications for reinforced concrete pipe.

24 in. ID, Class II, "B" wall. Concrete strength 4000 psi min. 3 in. reinforced wall thickness. Exterior surface coated with coal tar epoxy; some also with asphalt roof compound.

\section{Placement:}

Casks are set in a shallow trench. After sealing, tops are covered with galvanized sheeting, ther.

\section{Inside Configuration:}

Vertical placement in shallow trench. $\mathrm{J}-233$ waste placed in either 3-gallon or 15-gallon inner container sealed in concrete centered in 30-gallon drums.

\section{Content Data:}

See Table 3.36 which follows.

Major Radionuclides: Pu-238, U-233, Pu-239

0-0.250 R/hr gamma at contact with contained drums; lower but not measured values at cask surface. 
Table 3.36 Content of LANL Concrete Casks*

\begin{tabular}{|c|c|c|c|c|c|c|c|}
\hline Content Codes & Content Description & $\begin{array}{c}\text { Volume } \\
\mathrm{m}^{3}\end{array}$ & $\begin{array}{c}\text { Weight, } \\
\mathrm{Kq}\end{array}$ & $\begin{array}{l}\text { No. } \\
\text { Casks }\end{array}$ & $\begin{array}{c}\text { Pu-239 } \\
(\mathrm{g})\end{array}$ & $\begin{array}{l}p_{u-238} \\
(q)\end{array}$ & $\begin{array}{c}11-233 \\
(\mathrm{~g}) \\
\end{array}$ \\
\hline $\begin{array}{l}\text { A15, A16, A18 } \\
A 60\end{array}$ & $\begin{array}{l}\text { Combustibles - mixed } \\
\text { cellulosics, plastic } \\
\text { material, mixed paper, } \\
\text { plastic, rubber, other } \\
\text { combustibles }\end{array}$ & 84 & 107,562 & 84.5 & 43 & 1254.9 & 0 \\
\hline A17 & Rubber material & 17.1 & 19,990 & 15.5 & 0 & 122.1 & 0 \\
\hline A19 & $\begin{array}{l}\text { Mixed combustibles - } \\
\text { noncombustible }\end{array}$ & 40.8 & 47,880 & 38 & 0 & 1314.3 & 0 \\
\hline $\begin{array}{l}A 30, A 31, A 50, \\
A 52\end{array}$ & $\begin{array}{l}\text { Metal - equipment, metal } \\
\text { crucibles, scrap dies, other } \\
\text { scrap metal }\end{array}$ & 53 & 64,524 & 49.5 & 3 & 781.3 & 0 \\
\hline A55 & Filters - Filter Media & 4.5 & 11,170 & 8 & 0 & 113.5 & 0 \\
\hline A61 & Other noncombustibles & 92.8 & 135,600 & 107 & 250 & 2389.6 & 4,302 \\
\hline$A 25$ & Leached process residues & 1.6 & 2,521 & 2 & 3 & 21.2 & 0 \\
\hline A95 & Glass & 1.1 & 1,342 & 1 & $\underline{0}$ & 6.9 & 0 \\
\hline TOTAL & & 294.9 & 390,589 & 305.5 & 299 & 6003.8 & 4,302 \\
\hline
\end{tabular}

* Data taken from LANL stored TRU Waste Data, found in Appendix. 
Table 3.37

\begin{tabular}{lcccc} 
& Category & Number of Items & Total Volume & Ref. \\
\hline NTS & 55 -gallon drums & 633 & $134 \mathrm{~m}^{3}$ & 21 \\
\hline
\end{tabular}

\section{Description of Category:}

Cylindrical carbon steel drum.

Dimensions: 22.5 in. I0 $\times 33.25$ in. L, 24 in. OD $\times 35$ in. L (.478 in. wall)

Volume: $7.42 \mathrm{ft}^{3}$

\section{Placement:}

Above grade on asphalt pad. Drums are contained in cargo containers. Drums are stacked two high, 36 drums per tier. Container holds 72 drums. The cargo container is rectangular with $7 \mathrm{ft} 6$ in. minimum wide by $6 \mathrm{ft} 11-7 / 16 \mathrm{in}$. minimum high door. Outside dimensions: $8 \mathrm{ft} \times 8 \mathrm{ft} 6 \mathrm{in}$. high by $19 \mathrm{ft} \mathrm{10-1/2} \mathrm{in.} \mathrm{long.}$

\section{Inside Configuration:}

Loose waste, not compacted. Drum sealed with pressed cork gasket until 1980. In 1980, terpolymer sealant "sealant 7600" used. Both sealed with bolted closure ring.

\begin{tabular}{|c|c|c|c|c|c|}
\hline & $\begin{array}{c}\text { Waste } \\
\text { Description } \\
\end{array}$ & $\begin{array}{c}\text { No } \\
\text { Containers } \\
\end{array}$ & $\begin{array}{c}\text { Volume } \\
\left(\mathrm{m}^{3}\right)\end{array}$ & $\begin{array}{l}\text { Weight } \\
(\mathrm{kg}) \\
\end{array}$ & $\begin{array}{r}P u \\
\left(\mathrm{~g} / \mathrm{m}^{3}\right) \\
\end{array}$ \\
\hline $\begin{array}{l}\text { Combustibles } \\
\text { Process Solids } \\
\text { Metal Scrap }\end{array}$ & - paper, plastic, rags ${ }^{\star \star}$ & $\begin{array}{r}541 \\
64 \\
28\end{array}$ & $\begin{array}{c}114.7 \\
13.6 \\
6\end{array}$ & $\begin{array}{r}38,160 \\
7,053 \\
2,538\end{array}$ & $\begin{array}{l}24 \\
38.8 \\
10.1\end{array}$ \\
\hline
\end{tabular}

* Approximation based on Lawrence Livermore percentages for one year applied to total volume.

** A container is defined as "combustible" if greater than $10 \%$ by weight of the waste is combustible. 


\begin{tabular}{lcccc} 
& Category & Number of Items & Total Volume & Ref. \\
\hline NTS & Steel Boxes & 29 & $171 \mathrm{~m}^{3}$ & 21 \\
\hline
\end{tabular}

\section{Description of Category:}

Welded carbon steel boxes, painted white.

Dimensions: Three are $0.63 \mathrm{~m} \times 0.63 \mathrm{~m} \times 4.6 \mathrm{~m}$; Largest $2.43 \mathrm{~m} \times 2.43 \mathrm{~m} \times 3 \mathrm{~m}$.

Volume: Avg. $4.2 \mathrm{~m}^{3}$

\section{Placement:}

Stored inside cargo containers, placed on asphalt storage pad.

Cargo container is $3.7 \mathrm{~m} \times 2.4 \mathrm{~m} \times 2.6 \mathrm{~m}$. Further description found on preceeding page Table 3.30

\section{Inside Configuration:}

Waste is loose, may be wrapped in plastic. Welded shut, or sealed with terpolymer sealant and bolted.

\section{Content Data:}

Gloveboxes, equipment, lathes, machinery. Gloveboxes are sprayed with polyurethane foam and nonflammable paint.

\# combustible - 25

\# noncombustible - 4 
Table 3.39

\begin{tabular}{lcccc} 
& Category & Number of Items & Total Volume & Ref. \\
\hline ORNL & 30 -gallon drums & 101 & $17.2 \mathrm{~m}^{3}$ & 12 \\
\hline
\end{tabular}

\section{Description of Category:}

Cylindrical stainless steel or black iron drums. Dimensions: 19 in. ID $\times 29$ in. L

Volume: $6 \mathrm{ft}^{3}$ *

\# Stainless steel $=86$

\# $\mathrm{Bl}$ ack iron $=15$

* ORNL designated volume.

\section{Placement:}

Stored in concrete building, $85 \%$ below grade. Drums are stacked in vertical 1 ayers.

\section{Inside Configuration:}

Waste is loose, not compacted, usually bagged in plastic. Drums are sealed with neoprene gasket and bolted closure ring.

\section{Content Data:}

Contents are only somewhat segregated by combustible/noncombustible. Waste is 55\% combustible, 45\% noncombustible. All waste comes from laboratories. Consists mainly of paper, plastic, cloth, cans, process equipment, filters, tools, gloves, bottles, rags, silica gel. All read less than 200 $\mathrm{mrem} / \mathrm{hr}$. TRU activity only known for total. See Table 3.10. 
Table 3.40

\begin{tabular}{lcccc} 
& Category & Number of Items & Total Volume & Ref. \\
\hline ORNL & 55 -gallion drums & 1587 & $360.2 \mathrm{~m}^{3}$ & 12 \\
\hline
\end{tabular}

Description of Category:

Cylindrical stainless steel or black iron drums.

Dimensions: 22.5 in. ID $\times 33.25$ in. $L, 24$ in. $00 \times 36$ in. $L(0.478-i n$. wall $)$

Volume: $8 \mathrm{ft}^{3}$ *

\# Stainless steel $=1325$

\# Black iron $=262$

* ORNL designated volume.

Placement:

Stored in concrete building, $85 \%$ below grade. Drums are stacked in vertical layers.

\section{Inside Configuration:}

Waste is loose, not compacted, usually bagged in plastic. Drums are sealed with neoprene gasket and bolted closure ring.

\section{Content Data:}

Contents are only somewhat segregated by combustible/noncombustible. Waste is 55\% combustible, 45\% noncombustible. All waste comes from laboratories. Consists mainly of paper, plastic, cloth, cans, process equipment, filters, tools, gloves, bottles, rags, silica gel. All read less than 200 $\mathrm{mrem} / \mathrm{hr}$. TRU activity only known for total. See Table 3.10. 
Table 3.41

Category

ORNL Non-Standard Drums Number of Items

2
Total Volume

.67

\section{Description of Category:}

1 - 110 gal drum, consisting of two stainless steel 55-gal drums welded end-to-end Volume: $.43 \mathrm{~m}^{3}$

1 - overpack - mild steel drum Volume: $.24 \mathrm{~m}^{3}$

Placement:

Stored in underground building

Inside Configuration:

110 gal drum has plastic liner. Lid is clamped in place with bolted ring.

\section{Content Data:}

110 gal drum - 17 grams of U-233

Overpack - 28 grams of Pu-239

107 grams of $U-235$

50 percent combustible

Both drums less than $200 \mathrm{mR} / \mathrm{hr}$ at contact 
Table 3.42

\begin{tabular}{lcccc} 
& Category & Number of Items & Total Volume & Ref. \\
\hline SRP & 55 -gallon drums & 7065 & $1059 \mathrm{~m}^{3}$ & 14 \\
\hline
\end{tabular}

\section{Description of Category:}

Specification 17H: Cylindrical steel drum Dimensions: 22.5 in. ID $\times 33.25$ in. L, 24 in. $00 \times 35$ in. L (0.478-in wall) Volume: $7.62 \mathrm{ft}^{3} \star$

Since 1974 drums have been galvanized. Prior to that drums were painted.

Number drums galvanized $=3,532$

Number drums painted $=31$

* SRP designated

\section{Placement:}

Above ground on steel-base reinforced concrete pad. Newest pads are $150 \mathrm{ft} \times 60 \mathrm{ft}$. $01 \mathrm{der}$ pads can vary. A module consists of one pad which is completely filled with drums, boxes, and cement culverts. After a module is completed, the drums are covered with polyethelene plastic and $4 \mathrm{ft}$ layer of soil. Some stacking, two layers high, modules are covered with fish-net before plastic to resist tornadoes. Top soil is seeded.

\section{Inside Configuration:}

Since 1974 drums have contained polyethelene liner. Sealed with gasket and bolted closure ring. Waste can be loose, in plastic bags, buckets, or cans. Not compacted.

\section{Content Data:}

Specific contents are unknown. Since 1974 combustibles have been segregated from noncombustibles. Approximately $75 \%$ combustibles, $25 \%$ noncombustible. 
Table 3.43

\begin{tabular}{lcccc} 
& Category & Number of Items & Total Volume & Ref. \\
\hline SRP & steel boxes & 39 & 441 & 14 \\
\hline
\end{tabular}

\section{Description of Category:}

Welded carbon steel boxes.

Dimensions: Vary. Maximum allowable $6 \mathrm{ft} \times 8 \mathrm{ft} \times 12 \mathrm{ft}$

Volume: $\simeq 440 \mathrm{ft}^{3}$ (varies)

Lifting bails on top and side.

\section{Placement:}

Above ground on steel-base reinforced concrete pad. See description of pads and storage under SRP culverts (Table 3.42). Steel boxes are stored the same way.

\section{Inside Configuration:}

Sealed with gasket and bolted. Waste may be covered with plastic.

\section{Content Data:}

Specific contents unknown. Primarily large equipment, glove boxes. Less than $10 \%$ combustible. 
Table 3.44

\begin{tabular}{lcccc} 
& Category & Number of Items & Total Volume & Ref. \\
\hline SRP & cement boxes & 110 & $109 \mathrm{~m}^{3}$ & 14 \\
\hline
\end{tabular}

\section{Description of Category:}

Rectangular concrete boxes

Dimensions: $2 \mathrm{ft} \times 2 \mathrm{ft} \times 3 \mathrm{ft} 1 / 4 \mathrm{in}$. to $2.625 \mathrm{ft} \times 2.208 \mathrm{ft} \times 3.688 \mathrm{ft}$. Walls 4 -in to 5 -in thick.

Volume: $35 \mathrm{ft}^{3}$

\section{Placement:}

$\infty \quad$ Above ground on steel-base reinforced concrete pad. See description of pad under SRP culverts (Table 3.42). These boxes are stored the same way.

\section{Inside Configuration:}

Wall of cask is lined by $1 / 4 \mathrm{in.}$ carbon steel liner or case. Waste usually bagged in plastic.

\section{Content Data:}

Waste in these casks is from the Savannah River Laboratory. Includes a mixture of equipment, paper, plastic, gloves, and the usual laboratory-type of waste. Exact contents of each is unknown. Activity - varies widely; some contain $\mathrm{Cm}-244$ and $\mathrm{Cf}-252$. Most contain Pu-238. Radiation Level average $<200 \mathrm{mrem} / \mathrm{hr}$. Range -0 to $-500 \mathrm{mrem} / \mathrm{hr}$ at one foot. 
Table 3.45

\begin{tabular}{llccc} 
& Category & Number of Items & Total Volume & Ref. \\
\hline SRP & $\begin{array}{l}\text { Nonstandard } \\
\text { concrete boxes }\end{array}$ & 6 & $108 \mathrm{~m}^{3}$ & 14 \\
\hline
\end{tabular}

\section{Description of Category:}

Concrete boxes from Mound Facility and Los Alamos National Laboratory.

Dimensions: Vary. Largest $-11 \mathrm{ft} 6 \mathrm{in.} \mathrm{high,} 8 \mathrm{ft} 6 \mathrm{in.} \times 6 \mathrm{ft} 6 \mathrm{in}$. Weight - 15 tons

Concrete is 6-in. thick

\section{Placement:}

$\because \quad$ Above ground on steel-base reinforced concrete pad. See description of pad under SRP culverts (Table 3.42).

\section{Inside Configuration:}

Unknown.

\section{Content Data:}

Mostly large equipment. Exact contents unknown. Estimated $10 \%$ combustible. Total curies 235,000. Radiation level and activity for each box is unknown. All waste is contaminated with $\mathrm{Pu}-238$. 
Table 3.46

\begin{tabular}{|c|c|c|c|c|}
\hline & Category & Number of Items & Total Volume & Ref. \\
\hline SRP & concrete culverts & 243 & $1847 \mathrm{~m}^{3}$ & 14 \\
\hline
\end{tabular}

\section{Description of Category:}

Cylindrical concrete casks.

Dimensions: Diameter $-1.8 \mathrm{~m}$, height $2 \mathrm{~m}$, thickness $-0.0142 \mathrm{~m}$ weight - (cask only) 10-1/2 ton Volume: $8.21 \mathrm{~m}^{3}$

Grappling Hook on top, for lid only. Hooks on side

\section{Placement:}

Above ground on steel-base reinforced concrete pad. Newest pads are $150 \mathrm{ft} \times 60 \mathrm{ft}$. $01 \mathrm{der}$ pads can vary. A module consists of one pad which is completely filled with drums, boxes, and cement

culverts. After a module is completed, the culverts are covered with polyethelene plastic and $4 \mathrm{ft}$ layer of soil. No stacking of culverts. Top soil is seeded.

\section{Inside Configuration:}

Culverts contain either $1455-g a l l o n$ drums or 8 polyethene boxes. Cement lids are sealed in place with resin. Inside drums or boxes, waste is loose, but usually bagged in plastic. Approximately $75 \%$ of culverts contain drums, $25 \%$ contain boxes.

\section{Content Data:}

Specific contents are unknown. Much of the waste is known to be combustible. Combustibles have been segregated within drums since 1974 . Estimated $58 \%$ combustible, $42 \%$ noncombustible since 1974. Four cylinders are known to contain waste with $\mathrm{Cf}-252$ present. Individually one cylinder could exceed $200 \mathrm{mR} / \mathrm{hr}$; collectively they average $205 \mathrm{mR} / \mathrm{hr}$ and one classed as contact handled. Drums that are placed in the concrete culverts are those that are greater than $.5 \mathrm{Ci} / \mathrm{drum}$. 


\subsection{Remote-Handled - Summary}

Remote-handled wastes are stored at Los Alamos, Hanford, Oak Ridge, and Idaho National Engineering Laboratory. Table 3.47 shows the volume and TRU content of each site. Because remote-handled waste records are even more scarce than are contact-handled waste records, data on waste forms are usually estimated or not available. To get an idea of the types of waste forms, the reader is referred to Section 2.3 on currently generated remote waste forms. The data in that section were collected for approximately one year of output. The percentages of waste forms will be reasonably close to the percentages of remote-handled, storedwaste forms. In some cases the waste consists of spent fuel from testing and examination programs. The details on fuel type were not readily available.

Table 3.47 shows the total TRU elements contained in the remote waste. Table 3.48 shows a further breakdown of the TRU isotopes. For LANL and Hanford these totals are based upon typical isotopic composition of weapons grade plutonium. For Oak Ridge and INEL the totals of each isotope were provided by the site.

\subsection{Review of the RH Waste Categories at Storage Sites}

Table 3.49 summarizes the wastes categories at each site. These data come from the detailed tables that follow. A detailed breakdown of each category is found in Table 3.50 through 3.57. These data are presented in much the same manner as the previous contact-handled data. 
Table 3.47

Reported Inventories of Remote-Handled Transuranic Waste to Dec. $31,1980^{1}$

\begin{tabular}{|c|c|c|c|}
\hline $\begin{array}{l}\text { Storage } \\
\text { Site } \\
\end{array}$ & $\begin{array}{l}\text { Volume in } \\
\text { Storage }\left(\mathrm{m}^{3}\right) \\
\end{array}$ & $\begin{array}{l}\text { Mass of TRU } \\
\text { Elements }(\mathrm{kg})\end{array}$ & $\begin{array}{l}\text { Major Remote Generator } \\
\text { Shipping to this Site }\end{array}$ \\
\hline Hanford & 1261.1 & 5.2 & $\begin{array}{l}\text { Westinghouse Hanford } \\
\text { Company (HEDL) }\end{array}$ \\
\hline INEL & 17.8 & 0.064 & $\begin{array}{l}\text { Argonne National } \\
\text { Laboratory-East* }\end{array}$ \\
\hline LANL & 7.9 & 0.70 & \multirow{3}{*}{$\begin{array}{l}\text { Al1 Waste } \\
\text { Generated } \\
\text { On Site }\end{array}$} \\
\hline ORNL & 532.2 & 4.1 & \\
\hline TOTAL & 1819.5 & 9.36 & \\
\hline $\begin{array}{l}\star \text { Has be } \\
\text { Proces }\end{array}$ & $\begin{array}{l}\text { argest contr } \\
\text { Plant will }\end{array}$ & $\begin{array}{l}\text { r. Projecti } \\
\text { e largest ir }\end{array}$ & $\begin{array}{l}\text { low the Idaho Chemical } \\
\text { S. }\end{array}$ \\
\hline
\end{tabular}


Table 3.48

Major TRU Radionuclide Content in Remote-Handled Waste

(To December 31,1980 )

\begin{tabular}{|c|c|c|c|c|c|c|c|c|}
\hline \multirow[b]{2}{*}{ Isotope } & \multicolumn{2}{|c|}{ Hanford $^{8}, 19$} & \multicolumn{2}{|c|}{ INEL 20,22} & \multicolumn{2}{|c|}{ LANL $^{9}, 10$} & \multicolumn{2}{|c|}{$\mathrm{ORNL}^{12}$} \\
\hline & Grams & Curies & Grams & Curies & Grams & Curies & Grams & Curies \\
\hline$A m-241$ & 0 & 0 & 0 & 0 & 0 & 0 & 11.4 & 38.88 \\
\hline Am-243 & 0 & 0 & 0 & 0 & 0 & 0 & $<0.1$ & 1.48 \\
\hline$C f-252$ & 0 & 0 & 0 & 0 & 0 & 0 & $<0.1$ & 5.35 \\
\hline $\mathrm{Cm}-244$ & 0 & 0 & 0 & 0 & 0 & 0 & 14.92 & 1213 \\
\hline Pu-238 & 1.34 & $22.4^{\star}$ & 0.1 & 2.353 & 0.1 & $1.75^{\star}$ & 0 & 0 \\
\hline Pu-239 & 4205.4 & $259.8^{*}$ & 58.7 & 3.603 & 328.65 & $20.3^{\star}$ & 8.1 & 0.495 \\
\hline$P u-240$ & 255.28 & $62.7^{*}$ & 5.1 & 1.111 & 19.95 & $4.9 *$ & 1.0 & 0.215 \\
\hline Pu-241 & 13.44 & $1554.1^{*}$ & 0.6 & 61.49 & 1.05 & $121.45^{\star}$ & $<0.1$ & 0.05 \\
\hline$P u-242$ & 1.34 & $<0.1^{\star}$ & 0.3 & 0.1 & 0.1 & $<0.1^{\star}$ & 0 & 0 \\
\hline$U-233$ & 21.4 & 0.2 & 0.27 & $<0.1$ & 0 & 0 & 600 & 5.68 \\
\hline
\end{tabular}

* Calculated from total $\mathrm{Pu}$ reported using typical weapons grade percentages (Table 2.6). 
Table 3.49 Remote Handled Waste Categories At TRU Waste Storage Sites ( To December 31,1980 )

\begin{tabular}{|c|c|c|c|c|c|}
\hline Sites & $\begin{array}{l}\text { Flatbed } \\
\text { Rail } \\
\text { Car } \\
\end{array}$ & $\begin{array}{l}\text { Steel } \\
\text { Capsules, } \\
\text { Casks, or } \\
\text { Inserts }\end{array}$ & $\begin{array}{l}\text { Paint } \\
\text { Cans }\end{array}$ & $\begin{array}{l}30-g a l \\
\text { Drums }\end{array}$ & $\begin{array}{l}\text { Concrete } \\
\text { Cylinders }\end{array}$ \\
\hline HANFORD & & & $\begin{array}{l}\text { (In } \\
\text { caissons) }\end{array}$ & & \\
\hline $\begin{array}{l}\text { Vol. of one } \\
\text { container }\left(\mathrm{m}^{3}\right)\end{array}$ & varies & varies & 0.0043 & - & -- \\
\hline $\begin{array}{l}\text { \# of containers } \\
\text { Total Volume }\left(\mathrm{m}^{3}\right) \\
\text { SITE TOTAL VOLUME: }\end{array}$ & $\begin{array}{l}17 \text { cars } \\
1104 \\
1261.1 \mathrm{~m}^{3}\end{array}$ & $\begin{array}{l}45 \\
137\end{array}$ & $\begin{array}{l}4580 \\
20.1\end{array}$ & none & none \\
\hline $\begin{array}{c}\text { Curies of TRU Activ } \\
\text { Average } \\
\text { Range }\end{array}$ & $\begin{array}{l}\text { vity } \\
\text { Not Avail } \\
0-212 \mathrm{Ci} / \mathrm{can}\end{array}$ & $\begin{array}{l}\text { Not Avail } \\
0-.7 \mathrm{Ci} / \mathrm{Vol}\end{array}$ & $\begin{array}{l}0.43 \mathrm{Ci} / \mathrm{can} \\
0-6 \mathrm{Ci} / \mathrm{can}\end{array}$ & ln -- & -- \\
\hline
\end{tabular}

INEL

$\begin{array}{llllll}\text { Vol. of one } & & & & \\ \text { container }\left(\mathrm{m}^{3}\right) & -- & 0.14 & -- & 0.113 & -- \\ \text { \# of Containers } & \text { none } & 8 & \text { none } & 147 & \text { none } \\ \text { Total Volume }\left(\mathrm{m}^{3}\right) & -- & 1.2 & -- & 16.6 & -- \\ \text { SITE TOTAL VOLUME: } 17.8 \mathrm{~m}^{3} & & & & \\ \begin{array}{l}\text { Curies of TRU Activity } \\ \text { Average } \\ \text { Range }\end{array} & & & & \end{array}$

LANL

Vol, of one container $\left(\mathrm{m}^{3}\right)$

\# of containers none

Total Volume $\left(\mathrm{m}^{3}\right)$

SITE TOTAL VOLUME: $7.9 \mathrm{~m}^{3}$

Curies of TRU Activitya

Average

Range

a Many curies of non-TRU activity are usually present in remote-handled waste. Curies of non-TRU are not included.

b Some are found inside 30-gallon drums.

$c$ SRP has some which contain $C f-252$. The average radiation level is $205 \mathrm{mrem} / \mathrm{hr}$ and they are found in the contact-handled section of this report. Some individual cylinders may be remote-handled. 
Table 3.49 (Continued)

\begin{tabular}{cccc} 
Flatbed & Steel \\
Rail & Capsules, & & \\
Sites & Casks, or & Paint & $30-g a l$ Concrete \\
Car & Inserts & Cans & Drums Cylinders \\
\hline
\end{tabular}

ORNL

\begin{tabular}{|c|c|c|c|c|c|}
\hline container $\left(\mathrm{m}^{3}\right)$ & -- & varies & -- & -- & variesb \\
\hline $\begin{array}{l}\text { \# of containers } \\
\text { Total Volume }\left(\mathrm{m}^{3}\right) \\
\text { SITE TOTAL VOLUME: }\end{array}$ & $\begin{array}{l}\text { none } \\
-- \\
532.2 \mathrm{~m}^{3}\end{array}$ & $\begin{array}{l}32^{d} \\
3.4\end{array}$ & $\begin{array}{l}\text { none } \\
--\end{array}$ & -- & $\begin{array}{l}196^{d} \\
528.8\end{array}$ \\
\hline $\begin{array}{l}\text { Curies of TRU Activ } \\
\text { Average } \\
\text { Range }\end{array}$ & $t y^{a}$ & Not Av & & & Not Avail \\
\hline
\end{tabular}

SRP

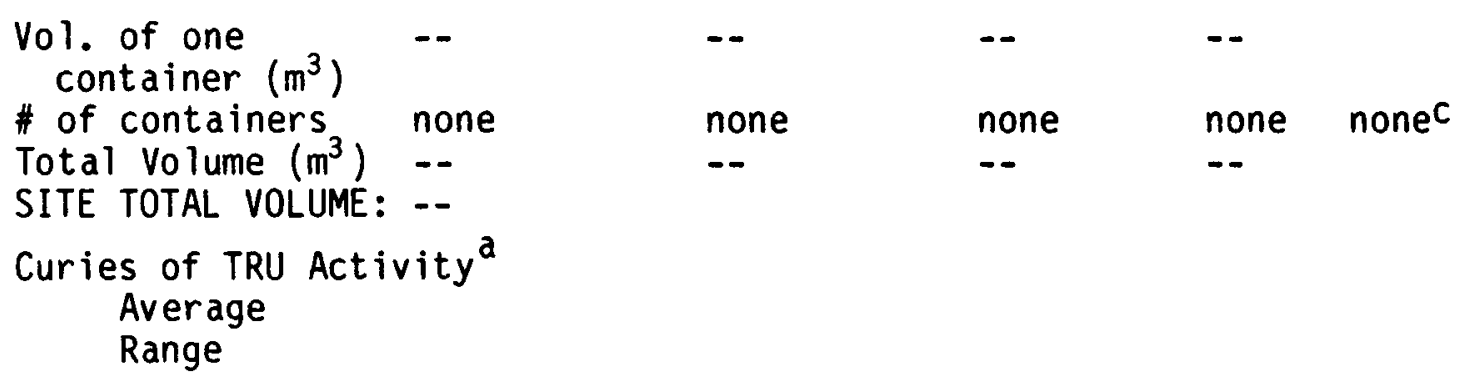

Total \# containers 17

$\begin{array}{llll}85 & 4580 & 147 & 202\end{array}$

TOTAL VOLUME $\quad 1104$

$\begin{array}{llll}141.6 & 20.1 & 16.6 & 536.7\end{array}$

TOTAL VOLUME ALL SITES: $1819 \mathrm{~m}^{3}$

a Many curies of non-TRU activity are usually present in remote-handled waste. Curies of non-TRU are not included.

b Some are found inside 30-gallon drums.

$c$ SRP has some which contain $\mathrm{Cf}-252$. The average radiation level is $205 \mathrm{mrem} / \mathrm{hr}$ so they are found in contact-handled section. Some individual cylinders may be remote-handled.

$d$ Some of these could be contact-handled due to decay of short-lived nuclides (fission by-products). 
Table 3.50

\begin{tabular}{cccc} 
Category & Number of Items & Total Volume & Ref. \\
\hline HANFORD paint cans & 4580 & $20.1 \mathrm{~m}^{3}$ & 7,8 \\
\hline
\end{tabular}

Description of Category:

Cylindrical tin paint can (1 galion)

Dimensions: $6-5 / 8$ in. diameter $\times 7-1 / 2$ in. high

Volume: $0.15 \mathrm{ft}^{3}$ (not including can)

Used in hot cell facility. Requires special truck for transportation, remote handling, and heavy shielding.

\section{Placement:}

Below ground in cylindrical concrete encasement known as a caisson. Caisson is 4 meters below - surface, dimensions: $8 \mathrm{ft}$. diameter $\times 10 \mathrm{ft}$. high. Cans are dumped into caisson down an angled steel chute, 0.9 meters in diameter. Five such caissons are in use, with no more anticipated.

\section{Inside Configuration:}

Waste is loose in paint cans. No liner, and no cement present. Surface may be contaminated. Lids are sealed with clip locks, but some are known to have broken open, spilling contents inside caisson. No compaction.

\section{Content Data:}

Contents include plastic, cloth, metal, paper, glass. Some irradiated fuel elements. See Table 2.20, which is a description of one years contents from Westinghouse Hanford, the major contributor to Hanford remote-handled waste. Activity - beta-gamma (Avg.) $=250.7 \mathrm{Ci} / \mathrm{ft}^{3}$ $\mathrm{Pu}_{\mathrm{u}}=6.38 \mathrm{~g} / \mathrm{ft}^{3}$ (Avg.) $\quad U=79.86 \mathrm{~g} / \mathrm{ft}^{3}$ Rad level $=7200 \mathrm{mR} / \mathrm{hr}$ Est. $=1000 \mathrm{mR} / \mathrm{hr}$ (unshielded) 
Table 3.51

\begin{tabular}{|c|c|c|c|c|}
\hline & ategory & Number of Items & Total Volume & Ref. \\
\hline HANFORD & $\begin{array}{l}\text { railroad flat } \\
\text { cars }\end{array}$ & 17 & $1104 \mathrm{~m}^{3}$ & 7,8 \\
\hline
\end{tabular}

\section{Description of Category:}

Large pieces of used equipment stored on flatbed railroad cars. Located in tunnel adjacent to Purex facility.

Dimensions: Car - $8 \mathrm{ft} \times 40 \mathrm{ft}$, Equipment - varies

\section{Placement:}

Tunnels are approximately $8 \mathrm{ft}$ below surface. Two tunnels. Equipment is stacked on cars usually with no additional containment.

\section{Inside Configuration:}

No liners, no concrete encasement.

\section{Content Data:}

Large pieces of equipment from Purex reprocessing facility. Example - dissolvers, condensors, piping, other metal equipment. Actixity - beta and gamma $=2.751 \mathrm{Ci} / \mathrm{ft}^{3}$ (Avg.) $\mathrm{Pu}=\langle 500 \mathrm{~g} \mathrm{per}$ load. Non-TRU isotopes include $\mathrm{Cs}^{137}, \mathrm{Sr}^{90}, \mathrm{Co}^{60}$. No estimate available of radiation level. 
Table 3.52

\begin{tabular}{ccccc} 
Category & Number of Items & Total Volume & Ref. \\
\hline HANFORD & nonstandard casks & 45 & $137 \mathrm{~m}^{3}$ & 7,8 \\
\hline
\end{tabular}

\section{Description of Category:}

Three types of nonstandard casks: (1) EBR-2 steel casks - carbon steel - lead. Dimensions: $30 \mathrm{ft} \times 5 \mathrm{ft}$ (2) Zirconium Hulls - Dimensions $30 \mathrm{ft} \times 5 \mathrm{ft} 10 \mathrm{in.} \mathrm{(3)} \mathrm{Concrete} \mathrm{casks} \mathrm{-} \mathrm{Dimensions:}$ $5 \mathrm{ft} 3$ in $\times 5 \mathrm{ft} 5$ in. $x 5 \mathrm{ft} 6$ in. All have special handling hooks. Thickness of steel and concrete unknown.

\section{Placement:}

Below ground in trenches, with $4 \mathrm{ft}$ of soil cover. No stacking, no additional containment.

\section{Inside Configuration:}

Waste can be loose or inside doubled 5-gallon cans. Liner present in steel casks, usually lead for additional radiation protection.

\section{Content Data:}

Hulls are leached fuel rods, from zirconium reactor fuel assemblies. Concrete casks contain liners from General Electric.

Steel cask - Activity beta and gamma $=40,000 \mathrm{Ci} /$ container (Avg.) less than $1.6 \mathrm{~g}$ Pu per container. Radition = not available

Concrete Cask - Activity - beta and gamma $=23,000 \mathrm{Ci} /$ containers (Avg.) 
Table 3.53

\begin{tabular}{|c|c|c|c|c|}
\hline & Category & Number of Items & Total Volume & Ref. \\
\hline INEL & $\begin{array}{l}30-g a l l o n \text { drums } \\
\text { (remote-handled) }\end{array}$ & 147 & $16.6 \mathrm{~m}^{3}$ & 20,22 \\
\hline
\end{tabular}

\section{Description of Category:}

30 gallon carbon steel drums.

Cylindrical, painted, 18 gauge.

Dimensions: 19 in. ID $\times 29$ in. L

Volume: $4 \mathrm{ft}^{3}$

\section{Placement:}

Placed in Intermediate Level TRU Storage Facility (ILTSF). ILTSF is carbon steel storage vault. Vault consists of $7.9 \mathrm{~m}$ vertical pipes, $61 \mathrm{~cm}$ and $40.1 \mathrm{~cm}$ in diameter. Pipes are enclosed in $1.5 \mathrm{~m}$ high earth shield, with a removable cement shield plug. Gasketed cover is used on the vault.

\section{Inside Configuration:}

Waste in 30-gallon drum with 100 mil rigid plastic liner. Waste usually contained in 3- or 5-gallon paint cans (steel) but may be loose. When loose is bagged in plastic. Drum is sealed with rubber gasket and bolted closure ring.

\section{Content Data:}

Majority of contents come from Argonne National Laboratory East. See Table 2.20 for one-year breakdown. Activity - $0.32 \mathrm{~g} \mathrm{Pu}-239 / \mathrm{drum}$ (ave.) - $0.03 \mathrm{~g} \mathrm{Pu-240/drum} \mathrm{(avg.)}$

Data on non-TRU activity not available.

Radiation level: Limits are greater than $200 \mathrm{mrem} / \mathrm{hr}$, less than $30 \mathrm{R} / \mathrm{hr}$.

Actual dose rates are: $0.4-50 \mathrm{R} / \mathrm{hr}$ at contact.

Average dose rate is $7.0 \mathrm{R} / \mathrm{hr}$ at contact, median dose rate is $4.0 \mathrm{R} / \mathrm{hr}$ at contact. 
Table 3.54

\begin{tabular}{lcccc} 
& Category & Number of Items & Total Volume & Ref. \\
\hline INEL & Steel Inserts & 8 & $1.2 \mathrm{~m}^{3}$ & 20,22 \\
\hline \hline
\end{tabular}

\section{Description of Category:}

Dimensions: Cylindrical stainless steel containers 12-3/4 in. $\times 72-i n$. long. Average volume - $5 \mathrm{ft}^{3}$

Lifting fixture is attached to cask.

\section{Placement:}

Placed in Intermediate Level TRU Storage Facility (ILTST). ILTSF is carbon steel storage vault. Vault consists of $7.9 \mathrm{~m}$ vertical pipes, $61 \mathrm{~cm}$ and $40.1 \mathrm{~cm}$ in diameter. Pipes are enclosed in $1.5 \mathrm{~m}$ high earth shield, with a removable cement shield plug. Gasketed cover is used on the vault.

\section{Inside Configuration:}

Waste inside carbon steel welded insert; usually inside plastic bags.

\section{Content Data:}

Waste is primarily associated with fuels examination and testing. Specific contents not available. Waste includes fuel assemblies, paper, gloves, plastic, labware, and small tools and equipment. Radioactivity Limits: $>200 \mathrm{mrem} / \mathrm{hr},<1000 \mathrm{R} / \mathrm{hr}$ at contact.

Actual dose rates $0.5-71.0 \mathrm{R} / \mathrm{hr}$ at $3 \mathrm{ft}$. Avg. $3 \mathrm{ft}$ dose $=16.6 \mathrm{R} / \mathrm{hr}$.

Average dose rate $=3.9 \mathrm{R} / \mathrm{hr}$ at $3 \mathrm{ft}$.

Radionuclides - see Table 3.48 . 
Table 3.55

\begin{tabular}{|c|c|c|c|c|}
\hline & Category & Number of Items & Total volume & Ref. \\
\hline LANL & Concrete $\mathrm{Cyl}$ inders & 6 & $7.9 \mathrm{~m}^{3}$ & 9,10 \\
\hline
\end{tabular}

Description of Category:

Stee 1 casks encased in concrete.

Overall: $0.61 \mathrm{~m} \times 4.4 \mathrm{~m}$, including lifting fixtures

Dimensions: Pipe - (2) one $0.23-0.30 \mathrm{~m} \mathrm{ID} \times 3.66 \mathrm{~m} \mathrm{~L}$ one $0.61 \mathrm{~m} 00 \times 4.27 \mathrm{~m} \mathrm{~L}$

Both pipes are sealed at one end with steel plate. Smaller pipe is centered in large pipe, partially enclosed in concrete.

Placement:

Casks stand vertically in individual shafts $0.76 \mathrm{~m}$ diameter $\times 4.2$ to $4.3-\mathrm{m}$ deep. Concrete is used to seal around the cask tops in the shafts. This is a very thin layer of concrete, leaving the cask retrievable without extensive removal methods.

Inside Configuration:

Hot cell wastes packaged in 3.8 \& paint cans, sealed in plastic container and bagged.

\section{Content Data:}

Includes hot-cell trash, wipes, glassware, small tools, absorbed liquids, and plastics. See Table 2.22 for description of one year. Activity - not available Beta-gamma activity - not available. Radiation level - 0.2-100 R/hr gamma at contact. 
Table 3.56

\begin{tabular}{|c|c|c|c|c|}
\hline & Category & Number of Items & Total Volume & Ref. \\
\hline ORNL & Concrete Casks & 196 & $528.8 \mathrm{~m}^{3}$ & 12 \\
\hline
\end{tabular}

\section{Description of Category:}

Reinforced Concrete Casks.

Dimensions: $1.27 \mathrm{~m}$ to $1.37 \mathrm{~m} \mathrm{ID} \times 2.13 \mathrm{~m}$ to $2.44 \mathrm{~m} \mathrm{~L}$

Wall thickness: (3 types used) $0.114 \mathrm{~m}, 0.152 \mathrm{~m}, 0.305 \mathrm{~m}$

\section{Placement:}

87 in unlined trenches with concrete pour over top; these are considered nonretrievable (buried). 183 in trenches without concrete pour.

13 in underground storage building. Use began in 1980.

\section{Inside Configuration:}

Waste placed in 3.8 \& metal paint cans. Sealed with metal lids, and heat sealed in polyethlene plastic.

\section{Content Data:}

Specific contents unknown. Table 2.23 shows the estimates for a one-year period. There are no sludges. Estimated metal content is $10 \%$ of the volume. Twenty casks contain large equipment items such as pumps and furnaces. Remainder is similar to Table 2.23. Activity - Avg. of $0.382 \mathrm{Ci} / \mathrm{m}^{3}$. Radiation level - was greater than $200 \mathrm{mR} / \mathrm{hr}$. Decay may have reduced this to less than $200 \mathrm{mR} / \mathrm{hr}$. 
Table 3.57

\begin{tabular}{llll} 
& Category & Number of Items & Total Volume \\
\hline ORNL & $\begin{array}{l}\text { Stainless Steel } \\
\text { Capsules }\end{array}$ & 32 & $3.4 \mathrm{~m}^{3}$ \\
\hline
\end{tabular}

Description of Category:

Stainless steel capsules

Dimensions vary: Smallest $-6-$ in. diameter $\times 30$ in. long

Largest (dia.) - 8-in. diameter $\times 100$ in. long

Largest $-5-i n$. diameter $\times 144$ in. long

\section{Placement:}

Placed in stainless steel wells; tops of wells are at ground level and surrounded by a concrete pad. Wells are closed with a concrete shielding plug.

\section{Inside Configuration:}

Capsules are welded or flanged and bolted. Waste is packaged on a case by case basis. May be bagged, canned, or loose.

\section{Content Data:}

Contents are mostly fuel elements which have been cut up for examination. Associated hardware may be present. See Table 2.23 for one year description. Activity - Avg of $0.382 \mathrm{Ci} / \mathrm{m}^{3}$. Radiation level - was $>200 \mathrm{mR} / \mathrm{hr}$. Could be less now due to beta-gamma products decay. 


\section{CHARACTERISTICS OF BURIED TRU WASTE}

Since weapons research began in the $1940^{\prime} \mathrm{s}$, land burial has been used to dispose of radioactive wastes. As used in this document, the term "buried," refers to those radioactive TRU wastes which have not been segregated into designated retrieval locations. In some cases, "stored" wastes are kept in underground trenches, and to the casual observer, these wastes would appear to be buried.

Prior to 1970, solid waste was buried such that no reliance was placed on the integrity of the container after burial. The containers were intended only to provide confinement for transportation to the burial ground. Most containers have been cardboard boxes, but wooden boxes, fiber drums, metal drums, plastic bags, and sprayed coatings have been used. Also, large pieces of equipment were often buried with no barrier, or encased in cement. Packages of waste contain rags, paper, gloves, supplies, tools, filters, and equipment, much the same as the stored wastes. Experimental retrieval studies have indicated that most of the original containers have deteriorated, but in many cases the plastic (bag) liners are still intact. ${ }^{24}, 25$ Trenches of various depths have been used with 12 feet being the most common depth. Three feet of soil is commonly used to cover buried waste, but this can also vary great $1 y$.

Because of these burial practices, a second large category of buried waste exists. Large quantities of soil have been contaminated not only from these buried wastes, but as a result of liquid waste disposal practices. Contaminated soil has resulted from liquids beinq sent directly to trenches, from cribs designed to hold or evaporate liquids, and from accidental leaks in tanks or pipes.

Table 4.1 shows the estimated volumes and TRU content of contaminated soil at the various sites. These totals cover a large range. The lower value is an estimate of the amount of soil probably contaminated 
to a TRU radioactivity level greater than $10 \mathrm{nCi} / \mathrm{g}$; the upper value is the amount of soil that might have to be handled if the waste were exhumed, depending on the exhumation method chosen. In cases where TRUcontaminated liquid waste was disposed of in soil (footnotes b, $c$, and $d$ of Table 4.1), the contaminated areas are more precisely known. Yet, it is not possible to determine how much soil must be disposed of (or processed) as TRU waste. Additional characterization data for disposal sites can be expected to define the subsurface contamination more accurately. 6

Table 4.2 shows the data that are available on the waste containers. Savannah River and Oak Ridge provide data other than general information because they began segregating some TRU waste during the burial period 1965 to 1970. H. M. Batchelder of EG\&G, Idaho, provided a table showing shipments of waste from Rocky Flats Plant, Colorado, to the INEL storage site from 1954 to 1970.26 The purpose of including this table is to give an indication of the types of packages that were buried in this early period. The total volumes do not match those of current inventories. The data are shown in Table 4.3. Table 4.4 gives the key dates affecting buried wastes at the various sites. Little more is known of the history or makeup of buried wastes. 
Table 4.1 Estimated Amounts of Buried TRU Waste

\begin{tabular}{|c|c|c|c|c|c|}
\hline Burial Site & $\begin{array}{c}\text { Solid waste } \\
\text { emplaced } \\
\left(\mathrm{m}^{3}\right)\end{array}$ & $\begin{array}{c}\text { Contaminated soil } \\
\text { from waste burial } \\
\left(\mathrm{m}^{3}\right)\end{array}$ & $\begin{array}{l}\text { Mass of TRU } \\
\text { elements } \\
(\mathrm{kg})\end{array}$ & $\begin{array}{c}\text { Radioactivity } \\
\text { (Ci) }\end{array}$ & Reference \\
\hline Hanford & 193,120 & $57,200-500,000^{b}$ & 605 & 160,000 & 8 \\
\hline INEL & 57,100 & $56,540-156,000$ & 357 & 178,000 & 22 \\
\hline LANL & 11,500 & $5,910-301,000^{C}$ & 13 & 49,200 & 10 \\
\hline NTS & 0 & 0 & 0 & 0 & 21 \\
\hline ORNL & 6,200 & $11,770-1,600,000$ & $5^{d}$ & 2,100 & 12 \\
\hline SRP & 31,000 & Up to 38,000 & 9 & 23,516 & 14 \\
\hline Sandia & 3 & & $<<1$ & & 1 \\
\hline TOTAL & $298,933 \mathrm{e}$ & $574,320-2,595,000$ & 1,000 & 412,816 & \\
\hline
\end{tabular}

a The volumes shown cover a range bounded by (1) the quantity of soil probably now contaminated to a level $>10 \mathrm{nCi} / \mathrm{g}$ and (2) the amount of soil that might have to be handled during exhumation of TRU waste until assay and TRU/LLW segregation are complete.

b Also at Hanford there are an estimated 2 to 7 million cubic meters of contaminated soil, containing less than $200 \mathrm{~kg}$ of plutonium resulting from early liquid waste disposal practices.

C In addition, there is an estimated $175-7,000 \mathrm{~m}^{3}$ of contaminated soil from liquid waste disposal, containing $\sim 0.14 \mathrm{~kg}$ of TRU elements.

d In addition, it is estimated that there is up to $1,000 \mathrm{~m}^{3}$ of contaminated soil from liquid waste disposal, containing $\sim 0.3 \mathrm{~kg}$ of TRU elements.

e There are some weapon accident residues $\left(<33 \mathrm{~m}^{3}\right)$ in plywood boxes at the Pantex Plant, Amarillo, TX, pending retrieval, identification, and shipment to NTS. 
Table 4.2 Buried Waste Categories at DOE Storage Sites Cardboard Boxes, Drums Miscellaneous

Concrete

Monoliths

\begin{tabular}{|c|c|c|c|c|c|}
\hline Sites & Vol $\left(\mathrm{m}^{3}\right)$ & TRU Activity (Curies) & Vol $\left(\mathrm{m}^{3}\right)$ & TRU Activity (Curies) & Reference \\
\hline INEL & 57,100 & $230,000 *$ & 0 & 0 & 22 \\
\hline LANL & 11,500 & $18,800 * \star$ & 0 & 0 & 10 \\
\hline NTS & 0 & 0 & 0 & 0 & 21 \\
\hline ORNL & 5,954 & $754 \star \star$ & 246 & $\begin{array}{l}\text { Included in box, } \\
\text { drum, and misc total }\end{array}$ & 12 \\
\hline Hanford & 193,120 & 256,562 & 0 & 0 & 8 \\
\hline SRP & 25,800 & 144,000 & 850 & $\begin{array}{l}\text { Included in box, } \\
\text { drum, and misc total }\end{array}$ & 14 \\
\hline TOTAL & 272,800 & $1,224,841$ & 850 & 253,879 & \\
\hline
\end{tabular}


Table 4.3 Summary of Containers of Solid Waste Shipped from Rocky Flats Plant to the INEL Storage Site, 1954 to $1970^{26}$

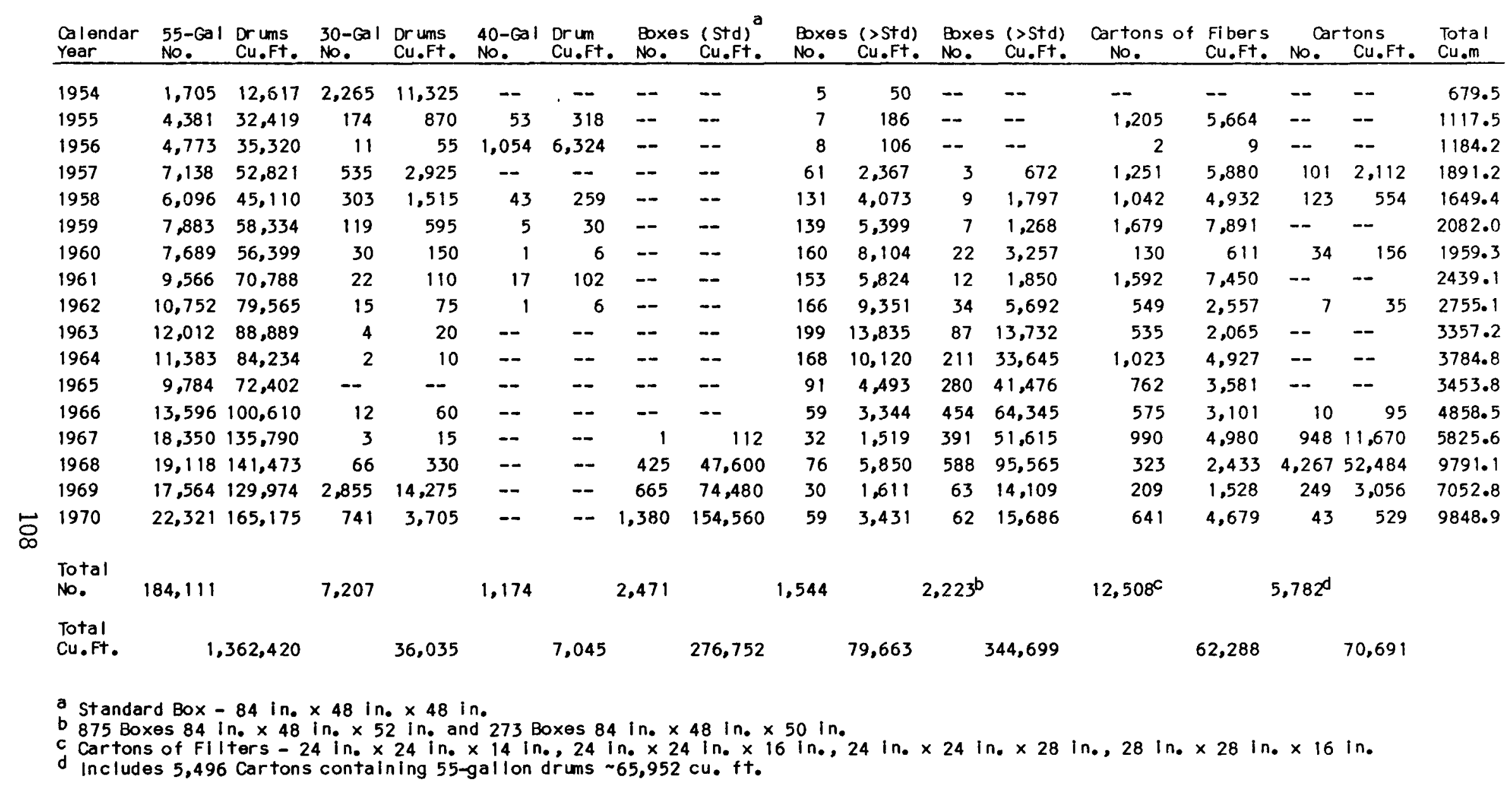


Table 4.4 SIgnificant Dates and Events Affecting Burled Wastes

TRU Burial

$1944-45$

$1952-56$

1958-67

$1970 *-74$

$1978-$

Reference

Hanford Hanford begins

solid waste

burial. TRU waste

is mixed with

beta- and gamma-

emitting waste.

INEL

1952-I NEL begins

solid waste
burial. TRU and

beta-gamma waste

are combined.

1967-Hanford ends

167-70 separate

solid waste burlal "alpha" trenches

in 300 Area (much are established

closer to City of Nov. 1970. TRU

Richland than waste segregated

current 200 Area). and stored.

1958 to 1963 INEL

Nov. 1970-TRU is

stacks waste

$4700 \mathrm{~m}^{3}$ of

drums, rather than stored.

merely dumping.

1963-They resume

dumping.

is retrleved

in experimen-

tal projects

and put in

1964-Separate

"alpha" trenches

storage.

established.

LANL

LANL begins solid

waste burlal. TRU waste is buried with beta-gammacontaminated

wasto. Plutonium

contaminated

liquid waste

disposed of in beds

or trenches.

1952-Pes idues from

liquid waste (TRU)

1968-Cemented Mid-1971. TRU mixed with cement

nate are mixed

segregated and

and burled.

into deep holes.

1956-Waste with

1 milligram pluto-

ni un or more bur-

led in special

shafts or holes.

ORNL ORNL begins solid

waste burlal. TRU

waste mixed with

beta-gamma. Exact

location not well

defined.

SRP

1953-SRP begins
solid waste
burlal. TRU and
beta-gamma conta-
minated waste.

1966-Li quid supernate is disposed

Oct. 1970-TRU stored.

of by shale frac-

waste in shale at

approx. $300 \mathrm{~m}$

below ground.

1965, SRP segre-

gates into

1974-Monollths dis

retrlevable and

continued. TRU

nonretr levable.

Waste greater than

- 1 cur le/package

gated and segre

minated waste.

put In concret

containers or

encapsul ated In

concrete monoliths

* 1970 AEC edict on storage of materials suspected to contain TRU radionuclides. 1973-AEC edict on $10 \mathrm{nCi} / \mathrm{g}$ I imit. 


\section{ADDITIONAL SOURCES OF TRU WASTE INFORMATION}

\subsection{Further Reports on Subjects Not Covered in This Document}

The information found in this section is not intended to represent an exhaustive compilation of TRU waste information. It represents a sampling of the most informative data that the author has seen while assembling this document. It focuses on information about the waste itself or the containers used to store the waste.

The DOE has a program in place to maintain a file of pertinent data on current and projected nuclear waste inventories. The 1981 report is "Spent Fuel and Radioactive Waste Inventories and Projections as of December 31, 1980," DOE/NE-0017, and is available from the ORNL. This is the official source for TRU waste data which is intended to be updated on an annual basis. In addition, the DOE also sponsors the Solid Waste Information Management Systems (SWIMS) at INEL. ${ }^{31}$ This system provides volumes and radionuclide data for all buried and stored solid radioactive wastes. The TWSO has published a Long Range Master Plan for the long-term disposal of TRU wastes. ${ }^{32}$ This document contains a comprehensive plan, covering R\&D, analysis, decision making, design, construction, operations, and decommissioning for all aspects of TRU waste management.

Anticipating the eventual retrieval and shipping of all its waste, EG\&G-Idaho has done a useful study on the nonradiological hazards of TRU waste. ${ }^{3}$ Another report done at INEL contains valuable data on retrieving buried wastes. The document was referred to earlier, and is titled "Initial Drum Retrieval, Final Report," by K. B. McKinley and J. D. MCKinney. ${ }^{25}$ Additional reports may be available from these authors. 
The TERA Advanced Services Corporation in Berkely, California, has published a very comprehensive document on waste container characteristics. It gives a detailed look at the containers which are presently approved by the Department of Transportation (DOT). It is titled, "Transuranic Contaminated Waste Container Characterization and Data Base," by B. G. Kniazewycz. ${ }^{34}$ Two summary reports that have been used extensively in the past are "Inventory and Sources of Transuranic Solid Waste," by the TERA Advanced Services Corporation, 27 and "Preliminary Evaluation of the Characteristics of Defense Transuranic Waste," by H. C. Shefelbine. ${ }^{15}$ This report was referred to earlier in this document. Parts of these documents are now somewhat outdated, but they do contain some information not included in this report. Finally, a document has been published by the Brookhaven National Laboratory that shows the relationship of TRU waste and high-level nuclear waste. The document gives inventories of both high-level and TRU waste. It is titled "Nuclear Waste Management Technical Support in the Development of Nuclear Waste Form Criteria for the NRC."135

\subsection{Information Available at Individual Sites}

All of the major storage sites, and many of the generating sites, have data in their data management (computer) centers. Sometimes a separate small computer is used, but often the system is part of a large site system.

Generally speaking, this report includes nearly all the data available on the specific contents of waste containers at storage sites. The one exception is the data system at INEL. They have much more information, and H. M. Batchelder should be contacted for specific needs. In the area of radionuclide data, almost all sites have additional specific data for certain years, packages, or locations. Also, most systems include data identifying the location of the waste at each of the sites. Concerning the origin of the waste, most readily available data were included in this report. A much more detailed picture could be 
Table 5.1 Description of Site Automated Data Systems

Site

Argonne National Laboratory-East

Hanford

INEL

\author{
Los Alamos \\ National \\ Laboratory \\ Mound Facility \\ Oak Ridge \\ National \\ Laboratory
}

Rocky. Flats

Plant

Savannah River Plant
Data System

Person to Contact

Most historial records available from H. M. Batchelder at INEL. Some recent data on computer at ANL-East.

Waste burial in the 200 Areas. Includes Battelle-PNL, UNC, and West inghouse-Hanford.

Printout included in Appendix.

Several systems which are currently being combined in one called NOMAD. Have data on all sites that ship to INEL and the SWIMS. Very extensive data.

New system that includes all LANL records. Data in appendix are typical of what is available.

Historical records are at INEL.

ORNL Solid Radioactive Waste Summary. Primary records on location, radionuclides, volumes, and location of generation.

B. M. Poremba

L. M. Cheevers

H. M. Batchelder

J. F. Warren

E. M. King

Extensive system on waste

forms and building of generation. Also have detailed data on individual packages.

System primarily records Volumes and location of burials, but has some data on stored radionuclide content, and location of generation. Called the COBRA system.

M. A. Aguilar

B. D. Helton 
obtained by conducting further specific research such as sampling studies. Table 5.1 shows the major sites, the name or description of their data system, and the person to contact to get more information on the kinds of data available.

Most of the sites have published, or are in the process of preparing, environmental impact statements or environmental assessments. These documents usually contain data on risk assessment which has not been covered in this document. They also include a large amount of information on the physical location and future impacts of the site on the environment. Waste management site plans contain similar information, and they are available at Hanford, INEL, RFP, LANL, SRP, and ORNL. A listing of each of these documents can be found in Appendix C. The Mound Facility program plan for D\&D is a similar document which explains site plans and impacts. ${ }^{36}$ 


\section{REFERENCES}

1. Department of Energy, Spent Fuel and Radioactive Waste Inventories and Projections as of December 31, 1980, D0E/NE-0017 (September 1981).

2. Department of Energy, Transuranic (TRU) Waste Management Program Strategy Document, DOE/AL/TRU-8002 (July 1980).

3. R. T. Jensen, Rockwell International, Rocky Flats Plant, Future Trends in Transuranic Waste Generation at Department of Energy Sites, RFP-3332, April 19, 1982.

4. L. M. Cheevers, Argonne National Laboratory-East, personal communication to R. T. Jensen, TRU Waste Systems Office (October 1981).

5. H. M. Batchelder, Idaho National Engineering Laboratory, 1981 and 10-Year Radioactive Waste Forecasts (January 1981).

6. C. W. Manry, Rockwell Hanford Operations, personal communication to J. G. Hilbig, Richland, Washington (April 16, 1981).

7. J. D. Anderson, Rockwell Hanford Operations, personal communication to R. T. Jensen, TRU Waste Systems Office (October 1981).

8. J. F. Albaugh, Rockwell Hanford Operations, personal communication to R. T. Jensen, Richland, Washington (February 23, 1982)

9. J. L. Warren, Los Al amos National laboratory, Stored TRU Waste Data, (See Appendix II), Los Al amos, New Mexico (December 1981).

10. J. L. Warren, Los Alamos National Laboratory, personal communication to R. T. Jensen, Los Alamos, New Mexico (March 12, 1982).

11. R. K. Blauvelt, Monsanto Research Corporation, personal communication to S. S. Martin, Miamisburg, Ohio, (June 1981). 
12. E. M. King, Oak Ridge National Laboratory, personal communication to S. S. Martin, Oak Ridge, Tennessee (June 24, 1981).

13. B. D. Helton, Savannah River Plant, Transuranic (TRU) Waste Characterization Study, (See Appendix II), Aiken, S.C. (August 25, 1981).

14. B. D. Helton, Savannah River Plant, personal communication to R. T. Jensen, Aiken, South Carolina (March 31, 1982).

15. H. C. Shefelbine, Sandia Laboratories, Preliminary Evaluation of the Characteristics of Defense Transuranic Waste, SAND78-1850, Albuquerque, NM (November 1978).

16. J. Case, INEL Naval Reactor Facility, personal communication to R. T. Jensen, Idaho Falls, Idaho (January 1982).

17. R. Mulkin, Los Alamos National Laboratory, Characterization of Transuranic Solid Wastes from a Plutonium Processing Facility, LA-5993-MS (June 1975).

18. W. D. Burch, Oak Ridge National Laboratory, personal communication to W. E. Clark, Oak Ridge, Tennessee, (October 1, 1970).

19. H. M. Batchelder, Idaho National Engineering Laboratory, unpublished data in WMIS System, (See Appendix II), Idaho Falls, Idaho (July 1981).

20. D. Gillas-Hiller, Reynolds Electric and Engineering Company, personal communication to R. T. Jensen, Las Vegas, Nevada (February 25, 1982).

21. J. D. Anderson and B. E. Poremba, Rockwell Hanford Operations, Summary of Radioactive Solid Waste Burials in the 200 Areas During 1980, RHO-LD-80-24 (April 27, 1981).

22. H. M. Batchelder, Idaho National Engineering Laboratory, personal communication to R. T. Jensen, Idaho Falls, Idaho (February 10, 1982). 
23. L. L. Luckett, Rockwell International-Rocky Flats Plant, personal communication to R. T. Jensen, Golden, Colorado (April 10, 1981).

24. J. L. Harness and J. D. McKinney, Containment of Transuranic Contamination at the Early Waste Retrieval Project, TREE-1061, EG\&G, Idaho, Inc., Idaho Falls, Idaho (January 1977).

25. K. B. McKinley and J. D. McKinney, Interim Drum Retrieval Final Report, TREE-1286, EG\&G, Idaho, Inc., Idaho Falls, Idaho (August 1978).

26. W. H. Lee to H. F. Soule, U.S. Atomic Energy Commission, personal communication, Rocky Flats Plant, Golden, Colorado (June 10, 1971).

27. TERA Advanced Services Corporation, Inventory and Sources of Transuranic Solid Waste, UCRL-13934, (August 1978).

28. Environmental Impact Statement for the Long-Term Management of Defense Transuranic Waste at the Idaho National Engineering Laboratory, U.S. Department of Energy, Idaho Operations Office, Idaho Falls, Idaho, (September 1981).

29. Alternative TRU Waste Management Strategies at Los Alamos National Laboratory, LA-8982-MS, Los Alamos National Laboratory, Los Alamos, New Mexico, (September 1981).

30. G. F. Molen, Savannah River Plant, Trends in the Generation of Plutonium Contaminated Solid Waste at the Savannah River Plant, DPST-80-448, Aiken, South Carolina (August 1980).

31. Solid Waste Information Management System (SWIMS) Data Summary Fiscal Year 1980, I00-10086(80), U.S. Department of Energy, Idaho Operations Office, Idahc Falls, Idaho (May 1981).

32. Long Range Master Plan for Defense Transuranic Waste Management, DOE-TRU-8201, U.S. Department of Energy, Albuquerque Operations Office, Albuquerque, New Mexico, (November 1982).

33. T. L. Clements, EG\&G-Idaho, personal communication to R. T. Jensen, Idaho Falls, Idaho (September 1981). 
34. B. G. Kniazewycz, Transuranic Contaminated Waste Container Characterization and Data Base, TERA Advanced Services Corporation, Berkeley, California (September 30, 1980).

35. Nuclear Waste Management Technical Support in the Development of Nuclear Waste Form Criteria for the NRC, Brookhaven National Laboratory, Upton, New York (February 1982).

36. Coordinated AWSPD (NEW) and OMA Decontamination and Decommissioning (CD\&D) Program Plan, Monsanto Research Corporation, Miamisburg, Ohio (April 30, 1981). 
APPENDIX A

\section{GLOSSARY OF ABBREVIATIONS}

AND ACRONYMS

\begin{tabular}{|c|c|c|}
\hline $\begin{array}{l}\text { AEC } \\
\text { ALO } \\
\text { APL } \\
\text { Am }\end{array}$ & $\begin{array}{l}- \\
\overline{-} \\
-\end{array}$ & $\begin{array}{l}\text { Atomic Energy Commission } \\
\text { Albuquerque Operations Office } \\
\text { (Bettis) Atomic Power Laboratory } \\
\text { Americium }\end{array}$ \\
\hline $\mathrm{CH}$ & - & Contact Handled \\
\hline $\begin{array}{l}\text { D\&D } \\
\text { DOE } \\
\text { DOT }\end{array}$ & - & $\begin{array}{l}\text { decontamination and decommissioning } \\
\text { Department of Energy } \\
\text { Department of Transportation }\end{array}$ \\
\hline $\begin{array}{l}\text { FRP } \\
\text { FFTF } \\
\text { FMEF }\end{array}$ & $\begin{array}{l}- \\
-\end{array}$ & $\begin{array}{l}\text { Fiberglass Reinforced Plastic } \\
\text { Fast Flux Test Facility } \\
\text { Fuels and Materials Examination Facility }\end{array}$ \\
\hline $\begin{array}{l}\text { HEPA } \\
\text { HEDL }\end{array}$ & - & $\begin{array}{l}\text { high efficiency particulate air } \\
\text { Hanford Engineering Development Laboratory }\end{array}$ \\
\hline $\begin{array}{l}\text { ICPP } \\
\text { INEL }\end{array}$ & - & $\begin{array}{l}\text { Idaho Chemical Processing Plant } \\
\text { Idaho National Engineering Laboratory }\end{array}$ \\
\hline $\begin{array}{l}\text { LANL } \\
\text { LLNL }\end{array}$ & - & $\begin{array}{l}\text { Los Alamos National Laboratory } \\
\text { Lawrence Livermore National Laboratory }\end{array}$ \\
\hline $\begin{array}{l}\text { N/A } \\
\text { N/R } \\
\text { NTS }\end{array}$ & - & $\begin{array}{l}\text { not available } \\
\text { not reported } \\
\text { Nevada Test Site }\end{array}$ \\
\hline $\begin{array}{l}\text { PNL } \\
\text { Pu }\end{array}$ & $\overline{-}$ & $\begin{array}{l}\text { Pacific Northwest Laboratory } \\
\text { Plutonium }\end{array}$ \\
\hline ORNL & - & Oak Ridge National Laboratory \\
\hline $\begin{array}{l}\text { R\&D } \\
\text { RFP } \\
\text { RH } \\
\text { RHO } \\
\text { RI-RFP }\end{array}$ & $\begin{array}{l}- \\
- \\
-\end{array}$ & $\begin{array}{l}\text { Research and Development } \\
\text { Rocky Flats Plant } \\
\text { Remote Handled } \\
\text { Rockwell Hanford Operations } \\
\text { Rockwell International - Rocky Flats Plant }\end{array}$ \\
\hline $\begin{array}{l}\text { SRL } \\
\text { SRP } \\
\text { SWIMS }\end{array}$ & - & $\begin{array}{l}\text { Savannah River Laboratory } \\
\text { Savannah River Plant } \\
\text { Solid Waste Information Management Systems }\end{array}$ \\
\hline $\begin{array}{l}\text { TRU } \\
\text { TWSO }\end{array}$ & - & $\begin{array}{l}\text { transuranic } \\
\text { Transuranic Waste Systems Office }\end{array}$ \\
\hline UNC & - & United Nuclear Company \\
\hline $\begin{array}{l}\text { WHC } \\
\text { WIPP }\end{array}$ & $\overline{-}$ & $\begin{array}{l}\text { West inghouse Hanford Company } \\
\text { Waste Isolation Pilot Plant }\end{array}$ \\
\hline
\end{tabular}




\section{APPENDIX B}

Tables Compiled from Computer Data at INEL, Los Alamos, Hanford, and Savannah River 
DATA COMPILED BY H. M. BATCHELDER

FROM EG\&G, IDAHO WMIS SYSTEM

TRANSURANIC STORAGE AREA (TSA) DATA(1)

9-71 thru 12-81

TABULATED BY CONTENT CODE

\begin{tabular}{|c|c|c|c|c|c|c|c|c|}
\hline \multirow[b]{2}{*}{ Generator * } & \multirow[b]{2}{*}{$\begin{array}{c}\text { Content } \\
\text { Code } \\
\end{array}$} & \multirow[b]{2}{*}{ Content Description } & \multicolumn{3}{|c|}{ Drums } & \multicolumn{3}{|c|}{ Boxes } \\
\hline & & & $\begin{array}{c}\text { Number } \\
\text { of } \\
\end{array}$ & $\begin{array}{c}\text { Volume } \\
\left(\mathrm{ft}^{3}\right) \\
\end{array}$ & $\begin{array}{l}\text { Weight } \\
\text { (1b) }\end{array}$ & $\begin{array}{c}\text { Number } \\
\text { of } \\
\end{array}$ & $\begin{array}{l}\text { Volume } \\
\left(\mathrm{ft}^{3}\right)\end{array}$ & $\begin{array}{l}\text { We ight } \\
\text { (1b) }\end{array}$ \\
\hline WMC & 0 & Not Recorded - Unknown & 18,972 & 146,033 & $4,066,585$ & & & \\
\hline RFO & 1 & First Stage Sludge & 7,750 & 58,353 & $3,804,908$ & & & \\
\hline RFO & 2 & Second Stage Sludge & 7,688 & 57,784 & $4,066,629$ & & & \\
\hline RFO & 3 & Organic Set Ups (oil solids) & 5,621 & 42,363 & $2,865,509$ & & & \\
\hline Rं́o & 4 & Special Set Ups (cement) & 1,237 & 9,650 & 724,090 & & & \\
\hline RFO & 5 & Evaporated Salts & 52 & 401 & 21,218 & 1 & 112 & 4,060 \\
\hline BET & 10 & Combustibles (rags, gloves, poly) & 912 & 6,968 & 173,533 & & & \\
\hline BET & 20 & Noncompressible, Noncombustible & 793 & 5,880 & 188,011 & & & \\
\hline BET & 30 & Solidified Grinding Sludge, etc. & 45 & 335 & 10,849 & & & \\
\hline BET & 40 & Solid Binary Scrap Powder, etc. & 172 & 1,728 & 83,706 & & & \\
\hline RFO & 90 & Dirt & 135 & 993 & 83,535 & & & \\
\hline RFO & 95 & Sludge & 23 & 169 & 8,880 & & & \\
\hline ALE & 102 & Absorbed Liquids & 26 & 205 & 6,154 & & & \\
\hline ALE & 104 & Alpha Hot Cell Waste & 190 & 745 & 18,333 & & & \\
\hline ALE & 105 & Empty Bottles \& Absorbent & 3 & 27 & 372 & & & \\
\hline
\end{tabular}

$\begin{array}{rr}\frac{(\mathrm{gm})}{24,958} & \frac{(\mathrm{gm})}{108} \\ 33,996 & 14,493 \\ 1,957 & 88 \\ 2,024 & 1 \\ 1,261 & 7 \\ 6 & 7 \\ 0 & 0 \\ 0 & 0 \\ 0 & 0 \\ 0 & 0 \\ 0 & 0 \\ 0 & 0 \\ 526 & 0 \\ 24 & 0 \\ 24 & 0\end{array}$


TRANSURANIC STORAGE AREA (TSA) DATA(1) (Cont'd)

Page 2 of 8 TABULATED BY CUNTENT CODE

\begin{tabular}{|c|c|c|c|c|c|c|c|c|c|c|}
\hline Generator* & $\begin{array}{c}\text { Content } \\
\text { Code } \\
\end{array}$ & Content Description & $\begin{array}{c}\text { Number } \\
\text { of } \\
\end{array}$ & $\begin{array}{c}\text { Drums } \\
\text { Volunie } \\
\left(f t^{3}\right)\end{array}$ & $\begin{array}{c}\text { Weight } \\
\text { (Ib) }\end{array}$ & $\begin{array}{c}\text { Number } \\
\text { of } \\
\end{array}$ & $\begin{array}{l}\text { Boxes } \\
\text { Volume } \\
\left(\mathrm{ft}^{3}\right) \\
\end{array}$ & $\begin{array}{l}\text { We ight } \\
\text { (1b) }\end{array}$ & $\begin{array}{r}\mathrm{Pu} \\
(\mathrm{gm})\end{array}$ & $\begin{array}{c}A m \\
(\mathrm{gm})\end{array}$ \\
\hline INEL & 150 & Laboratory Waste & 20 & 138 & 3,980 & & & & 444 & 0 \\
\hline INEL & 151 & Solidified Fuel Sludge & 2 & 8 & 700 & & & & 6 & 0 \\
\hline INEL & 152 & Pu Neutron Sources & 8 & 58 & 1,470 & & & & 221 & 0 \\
\hline INEL & 153 & Combustible Lab Waste & 28 & 116 & 1,970 & & & & 7 & 0 \\
\hline INEL & 154 & Sample Fuel & 2 & 14 & 800 & & & & 0 & 0 \\
\hline INEL & 155 & TRU Scrap & 5 & 36 & 545 & 1 & 112 & 150 & 2 & 0 \\
\hline INEL & 156 & Chem Cell Rip-out & & & & 7 & 896 & 18,550 & 9 & 0 \\
\hline $\mathrm{BCL}$ & 200 & Americium Sources & 1 & 7 & 50 & & & & 0 & 0 \\
\hline $\mathrm{BCL}$ & 201 & Noncombustible Solids & 54 & 397 & 17,576 & & & & 219 & 0 \\
\hline $\mathrm{BCL}$ & 202 & Combustible Solids & 8 & 59 & 1,374 & & & & 16 & 0 \\
\hline $\mathrm{BCL}$ & 203 & Paper, Metals, Glass & 26 & 191 & 5,429 & & & & 83 & 0 \\
\hline $\mathrm{BCL}$ & 204 & Solidified Solutions & 28 & 206 & 15,801 & & & & 10 & 0 \\
\hline RFO & 241 & Americium Process Residue & 120 & 897 & 43,997 & & & & 150 & 0 \\
\hline RFO & 290 & Sludge, Filter & 1 & 7 & 145 & & & & 14 & 0 \\
\hline RFO & 292 & Cemented Sludge & 275 & 2,022 & 73,136 & & & & 6,496 & 2 \\
\hline
\end{tabular}


TRANSURANIC STORAGE AREA (TSA) DATA(1) (Cont'd) TABULATED BY CONTENT CODE

\begin{tabular}{|c|c|c|}
\hline Generator & $\begin{array}{c}\text { Content } \\
\text { Code } \\
\end{array}$ & Content Description \\
\hline RFO & 300 & Graphite \\
\hline RFO & 301 & Graphite Cores \\
\hline RFO & $302 \star \star$ & Benelex and Plexiglass \\
\hline RFO & 310 & Graphite Scarfings \\
\hline RFU & 311 & Graphite Heels \\
\hline RFO & 320 & Tantalum \\
\hline RFO & 330 & Paper and Rags - Dry \\
\hline RFO & 335 & Filters, Absolute $\left(8^{\prime \prime} \times 8^{\prime \prime}\right)$ \\
\hline RFO & 336 & Paper and Rags - Moist \\
\hline RFO & 337 & Plastics, Teflon, Wash, PVC \\
\hline RFO & 338 & Insulation and CWS Filter Media \\
\hline RFO & 339 & Leaded Rubber Gloves and Aprons \\
\hline RFO & 360 & Insulation \\
\hline RFO & 361 & Insulation Heel \\
\hline
\end{tabular}

\begin{tabular}{rrr}
$\begin{array}{c}\text { Number } \\
\text { of }\end{array}$ & $\begin{array}{r}\text { Volume } \\
\left(\mathrm{ft}^{3}\right)\end{array}$ & $\begin{array}{r}\text { We ight } \\
(1 \mathrm{~b})\end{array}$ \\
\hline $\begin{array}{r}8,722 \\
33\end{array}$ & 243 & 300,437 \\
19 & 140 & 8,606 \\
16 & 118 & 3,922 \\
6 & 55 & 1,9987 \\
347 & 2,552 & 77,352 \\
5,074 & 37,783 & 930,070 \\
110 & 809 & 16,912 \\
7,393 & 54,473 & $1,457,785$ \\
2,142 & 15,904 & 365,354 \\
253 & 1,860 & 41,884 \\
656 & 4,824 & 222,973 \\
239 & 1,761 & 34,278 \\
1 & 11 & 411 \\
& &
\end{tabular}

\begin{tabular}{|c|c|c|c|c|}
\hline $\begin{array}{c}\text { Number } \\
\text { of } \\
\end{array}$ & $\begin{array}{l}\text { Boxes } \\
\text { Volume } \\
\left(\mathrm{ft}^{3}\right)\end{array}$ & $\begin{array}{l}\text { Weight } \\
\text { (Ib) }\end{array}$ & $\begin{array}{c}\mathrm{Pu} \\
\text { (gm) }\end{array}$ & $\begin{array}{c}\text { An } \\
(\mathrm{gm})\end{array}$ \\
\hline & & & 11,712 & 0 \\
\hline & & & 418 & 0 \\
\hline \multirow[t]{2}{*}{16} & 1,792 & 59,460 & 68 & 0 \\
\hline & & & 81 & 0 \\
\hline \multirow[t]{2}{*}{1} & 112 & 1,940 & 1,140 & 0 \\
\hline & & & 10,422 & 34 \\
\hline \multirow[t]{2}{*}{1,201} & 134,344 & $2,212,390$ & 17,758 & 1,008 \\
\hline & & & 215 & 7 \\
\hline 9 & 1,008 & 18,160 & 2,689 & 51 \\
\hline 9 & 1,008 & 18,080 & 1,892 & 82 \\
\hline \multirow[t]{2}{*}{78} & 8,736 & 153,890 & 6,501 & 0 \\
\hline & & & 16,122 & 16 \\
\hline \multirow[t]{2}{*}{1} & 112 & 1,860 & 217 & 0 \\
\hline & & & 199 & 0 \\
\hline
\end{tabular}


TRANSUR'ANIC STORAGE AREA (TSA) DATA(1) (Cont'd) TABULATEU BY CONTENT CODE

\begin{tabular}{|c|c|c|c|c|c|c|c|c|c|c|}
\hline \multirow[b]{2}{*}{ Gener a tor* } & \multirow[b]{2}{*}{$\begin{array}{l}\text { Content } \\
\text { Code }\end{array}$} & & \multicolumn{3}{|c|}{ Drums } & \multicolumn{3}{|c|}{ Boxes } & \multirow[b]{2}{*}{$\begin{array}{c}\rho_{u} \\
(\mathrm{gm}) \\
\end{array}$} & \multirow[b]{2}{*}{$\begin{array}{c}\text { Am } \\
(\mathrm{gm}) \\
\end{array}$} \\
\hline & & Content Description & $\begin{array}{c}\text { Number } \\
\text { of } \\
\end{array}$ & $\begin{array}{l}\text { Volume } \\
\left(\mathrm{ft}^{3}\right) \\
\end{array}$ & $\begin{array}{l}\text { Weight } \\
\text { (1b) }\end{array}$ & $\begin{array}{c}\text { Number } \\
\text { of } \\
\end{array}$ & $\begin{array}{l}\text { Volume } \\
\left(\mathrm{ft}^{3}\right) \\
\end{array}$ & $\begin{array}{l}\text { We ight } \\
\text { (1b) }\end{array}$ & & \\
\hline RFO & 370 & Crucible, Leco & 34 & 250 & 12,199 & 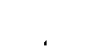 & & & 245 & 0 \\
\hline RFO & 371 & Brick, Fire & 958 & 7,048 & 346,619 & 24 & 2,688 & 54,760 & 3,703 & 0 \\
\hline RFO & 372 & Grit & 5 & 37 & 2,220 & & & & 21 & 0 \\
\hline RFO & 374 & Black top, Concrete, Dirt, and Sand & 1,097 & 8,067 & 428,348 & 46 & 5,152 & 148,420 & . 714 & 2 \\
\hline RFO & 375 & 0il Dri Residues from Incinerator & 14 & 103 & 4,953 & & & & 91 & 0 \\
\hline RFO & 376 & Cement Insul. and Filter Media & 1,049 & 7,714 & 210,802 & 7 & 784 & 16,733 & 23,650 & 128 \\
\hline RFO & 391 & Crucible and Sand & 19 & 140 & 6,510 & & & & 718 & 91 \\
\hline RFO & 392 & Sand, Slag, and Crucibles & 7 & 74 & 2,947 & & & & 1,233 & 0 \\
\hline RFO & 393 & Sand, Slag, and Crucible Heels & 20 & 147 & 4,494 & & & & 3,527 & 0 \\
\hline RFO & 410 & Molten Salts & 22 & 162 & 6,637 & & & & 233 & 21 \\
\hline RFO & 411 & Electrorefining Salt & 2 & 15 & 475 & & & & 24 & 0 \\
\hline RFO & 412 & Gibson Salts & 1 & 7 & 250 & & & & 21 & 0 \\
\hline RFO & 416 & Zinc Manganese Alloy Metal & 1 & 7 & 484 & & & & 170 & 31 \\
\hline RFO & 420 & Ash, Incinerator (virgin) & 9 & 66 & 3,421 & & & & 480 & 0 \\
\hline RFO & 421 & Heels, Ash ( 2 percent $G / G$ ) & 101 & 743 & 21,451 & & & & 17,765 & 0 \\
\hline RFO & 422 & Soot & 15 & 110 & 3,170 & & & & 807 & 0 \\
\hline
\end{tabular}


TRANSURANIC STORAGE AREA (TSA) DATA(1) (Cont'd) IABULATED BY CONTENT CODE

\begin{tabular}{|c|c|c|c|c|c|c|c|c|c|c|}
\hline Gener a tor* & $\begin{array}{l}\text { Content } \\
\text { Code }\end{array}$ & Content Description & $\begin{array}{c}\text { Number } \\
\text { of }\end{array}$ & 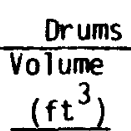 & $\begin{array}{l}\text { We ight } \\
\text { (1b) }\end{array}$ & $\begin{array}{c}\text { Number } \\
\text { of } \\
\end{array}$ & $\begin{array}{l}\text { Boxes } \\
\text { Volume } \\
\left(\mathrm{ft}^{3}\right)\end{array}$ & $\begin{array}{l}\text { Weight } \\
\text { (lb) }\end{array}$ & $\begin{array}{c}\mathrm{Pu} \\
(\mathrm{gm}) \\
\end{array}$ & $\begin{array}{c}\text { Am } \\
(\mathrm{gm}) \\
\end{array}$ \\
\hline RFO & 425 & Fluid Bed Ash & 8 & 59 & 3,134 & & & & 3 & 0 \\
\hline RFO & 430 & Resin, Ion Column Un leached & 29 & 266 & 11,528 & & & & 2,716 & 0 \\
\hline RFO & 431 & Resin, Leached & 6 & 59 & 2,387 & & & & 263 & 0 \\
\hline RFo & 432 & Resin, Leached and Cemented & 229 & 1,684 & 62,616 & & & & 7,158 & 82 \\
\hline RFO & 440 & Glass & 1,098 & 8,359 & 255,285 & 1 & 112 & 2,290 & 5,731 & 26 \\
\hline RFO & 441 & Raschig Rings, Unleached & 1,573 & 11,575 & 306,024 & & & & 12,453 & 9 \\
\hline RFO & 442 & Raschig Rings, Leached & 853 & 6,276 & 155,544 & & & & 1,819 & 14 \\
\hline RFO & 460 & washables, Rubber, Plastics & 6 & 67 & 2,813 & & & & 81 & 0 \\
\hline RFo & 463 & Gloves, Drybox & 53 & 510 & 19,533 & & & & 759 & 0 \\
\hline RFO & $464 \star \star$ & Plexiglass and Benelex & 48 & 364 & 12,971 & & & & 90 & 0 \\
\hline RFo & 480 & Metal Scrap (Non SS) & 2,117 & 16,013 & 536,508 & 3,116 & 347,214 & $9,016,490$ & 35,038 & 73 \\
\hline RF0 & 481 & Metal, Leached (Non SS) & 842 & 6,192 & 249,048 & 1 & 112 & 3,080 & 15,949 & 11 \\
\hline RFo & 490 & Filters, CWS & 58 & 460 & 11,626 & 717 & 80,304 & $1,357,240$ & 9,504 & 17 \\
\hline MND & 801 & Kags, Paper. Wood, etc. & 31 & 228 & 3,601 & & & & 28 & 0 \\
\hline MNO & 802 & Dry Box Gloves and O-Rings & 110 & 817 & 13,725 & & & & 394 & 0 \\
\hline
\end{tabular}


TRANSURANIC STORAGE AREA (TSA) DATA(1) (Cont'd)

Page 6 of 8 TABULATED BY CONTENT CODE

\begin{tabular}{|c|c|c|}
\hline Generator* & $\begin{array}{l}\text { Content } \\
\text { Code } \\
\end{array}$ & Content Description \\
\hline MND & 803 & Metal, Equip., Pipes, Valves, etc \\
\hline MND & 804 & $\begin{array}{l}\text { Plastic, Tygon, Manipulator } \\
\text { Boots, etc. }\end{array}$ \\
\hline MND & 805 & Asbes tos Filters \\
\hline MND & 810 & Giass, Flasks, Sample Vials, etc \\
\hline MND & 811 & Evaporator and Dissolver Sludge \\
\hline MND & 813 & Glass Filters and Fiberglass \\
\hline MND & 814 & Contaminated Mercury \\
\hline MND & 824 & Equipment Boxes \\
\hline MND & 825 & Equipment \\
\hline MND & 826 & Equipment Boxes-Combustible \\
\hline MND & 834 & High Level Acid \\
\hline MND & 835 & High Level Caustic \\
\hline MND & 836 & High Level Sludge/Cement \\
\hline MND & 838 & $<10 n C i / g m$ Noncombustible \\
\hline
\end{tabular}

\begin{tabular}{|c|c|c|c|c|c|}
\hline \multicolumn{3}{|c|}{ Drums } & \multicolumn{3}{|c|}{ Boxes } \\
\hline $\begin{array}{l}\text { Number } \\
\text { of }\end{array}$ & $\begin{array}{l}\text { Volume } \\
\left(\mathrm{ft}^{3}\right)\end{array}$ & $\begin{array}{l}\text { We ight } \\
\text { (1b) }\end{array}$ & $\begin{array}{c}\text { Number } \\
\text { of } \\
\end{array}$ & $\begin{array}{l}\text { Volume } \\
\left(\mathrm{ft}^{3}\right) \\
\end{array}$ & $\begin{array}{l}\text { Weight } \\
\text { (lb) }\end{array}$ \\
\hline 160 & 1,185 & 25,916 & & & \\
\hline 210 & 1,558 & 23,522 & & & \\
\hline 34 & 252 & 4,947 & & & \\
\hline 13 & 96 & 2,124 & & & \\
\hline 4 & 29 & 668 & & & \\
\hline 3 & 22 & 364 & & & \\
\hline 2 & 15 & 268 & & & \\
\hline & & & 201 & 34,888 & 431,453 \\
\hline 169 & 1,291 & 32,935 & 1 & 121 & 1,809 \\
\hline 2 & 16 & 772 & 7 & 743 & 13,068 \\
\hline 901 & 6,626 & 381,729 & & & \\
\hline 1,486 & 11,060 & 574,546 & & & \\
\hline 3,760 & 27,957 & $2,363,946$ & & & \\
\hline 1 & 7 & 335 & & & \\
\hline
\end{tabular}

\begin{tabular}{|c|c|}
\hline $\begin{array}{c}\mathrm{Pu} \\
(\mathrm{gm})\end{array}$ & $\begin{array}{c}\text { Am } \\
(\mathrm{gm})\end{array}$ \\
\hline 1,296 & 0 \\
\hline 265 & 0 \\
\hline 220 & 0 \\
\hline 96 & 0 \\
\hline 39 & 0 \\
\hline 29 & 0 \\
\hline 111 & 0 \\
\hline 781 & 0 \\
\hline 231 & 0 \\
\hline 1 & 0 \\
\hline 118 & 0 \\
\hline 114 & 0 \\
\hline 16 & 0 \\
\hline 0 & 0 \\
\hline
\end{tabular}




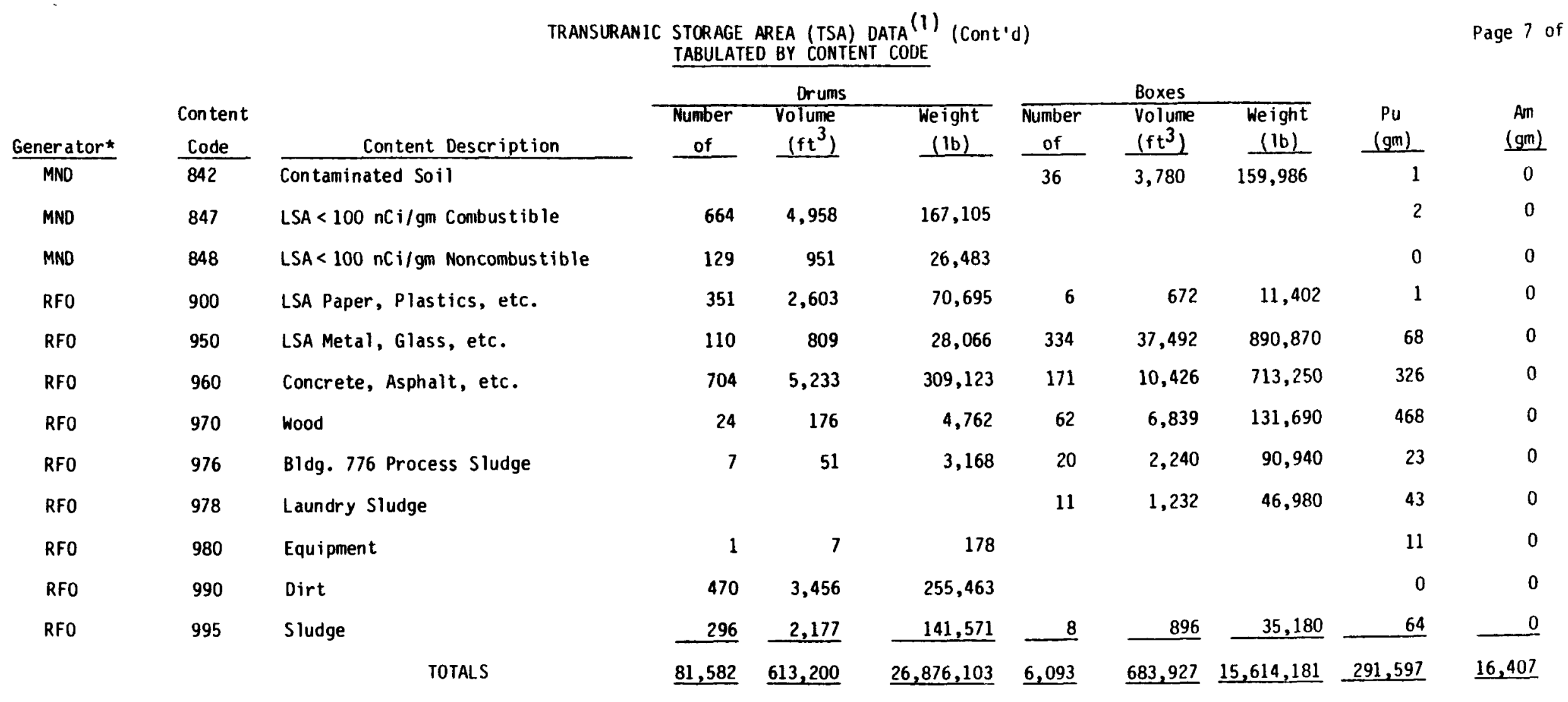


TRANSURANIC STORAGE AREA (TSA) DATA(1) (Cont'd)

Page 8 of 8

* Gener ator Acronyms

ALE Argonne National Laboratory-East

$\mathrm{BCL}$ Battelle Columbus Laboratories

BET Bettis Atomic Power Laboratory

INEL Idaho National Engineering Laboratory

MND Mound Laboratories

RFO Rocky Flats Operations (Plant)

WMC Waste Management Complex - Retrieved Drums

** SEE ALSO: Content Code $302 /$ Content Code 464

(1) Content code data is unavailable for the first year of TSA operation (11/70-9/71). 
DATA COMPILED BY J. F. WARREN FROM

LOS ALAMOS NATIONAL LABORATORY

WASTE MANAGEMENT SYSTEM

\begin{tabular}{|c|c|c|c|c|c|c|c|c|c|c|c|c|}
\hline \multirow{2}{*}{ WASTE CODE } & \multicolumn{4}{|c|}{ PACKAGES, VOLUME, AND WEIGHT } & \multicolumn{5}{|c|}{ RADIONUCL IDE CONTENT (GRAMS) } & \multicolumn{3}{|c|}{ PACKAGE RADIATION (mr/hr) } \\
\hline & Package & $\begin{array}{l}\text { Number of } \\
\text { Packages }\end{array}$ & Vol une $\left(\mathrm{m}^{3}\right)$ & Weight $(\mathrm{kg})$ & PU-239* & $\mathrm{PU}-238^{\star *}$ & AM-241 & U-233 & Other & High & Low & Average \\
\hline $\begin{array}{l}\text { Al0 } \\
\text { Graphite }\end{array}$ & $\begin{array}{l}30 \text { gal drum } \\
55 \text { gal drum } \\
\text { FRP box } \\
\text { Culvert a } \\
\text { Other }\end{array}$ & 1.0 & 0.2 & 70.3 & 8.7 & & & & & 1.0 & 1.0 & 1.0 \\
\hline Subtotal: & & 1.0 & 0.2 & 70.3 & 8.7 & & & & & & & \\
\hline $\begin{array}{l}\text { A15 } \\
\text { Mixed Cellulosics }\end{array}$ & $\begin{array}{l}30 \text { gal drum } \\
55 \text { gal drum } \\
\text { FRP box } \\
\text { Culvert a } \\
\text { 0ther }\end{array}$ & $\begin{array}{r}124.0 \\
222.0 \\
11.0\end{array}$ & $\begin{array}{l}12.5 \\
44.1 \\
11.1\end{array}$ & $\begin{array}{r}3862.0 \\
11730.0 \\
14140.0\end{array}$ & $\begin{array}{r}99.3 \\
705.0\end{array}$ & $\begin{array}{r}1.0 \\
17.0 \\
240.8\end{array}$ & & & & $\begin{array}{r}4.0 \\
18.0 \\
12.0\end{array}$ & $\begin{array}{l}1.0 \\
1.0 \\
1.0\end{array}$ & $\begin{array}{l}1.1 \\
1.3 \\
1.7\end{array}$ \\
\hline Subtota 1: & & 357.0 & 67.7 & 29732.0 & 804.3 & 258.8 & & & & & & \\
\hline $\begin{array}{l}\text { Al6 } \\
\text { Plastic Material }\end{array}$ & $\begin{array}{l}30 \text { gal drum } \\
55 \text { gal drum } \\
\text { FRP box } \\
\text { Culvert a } \\
\text { other }\end{array}$ & $\begin{array}{r}9.0 \\
19.0 \\
\\
2.5\end{array}$ & $\begin{array}{l}1.0 \\
3.8 \\
\\
2.4\end{array}$ & $\begin{array}{r}244.8 \\
1017.0 \\
3142.0\end{array}$ & $\begin{array}{l}21.6 \\
81.2\end{array}$ & 112.2 & & & & $\begin{array}{r}1.0 \\
150.0 \\
5.0\end{array}$ & $\begin{array}{l}0.0 \\
1.0 \\
0.0\end{array}$ & $\begin{array}{r}0.1 \\
11.1 \\
1.7\end{array}$ \\
\hline Subtotal: & & 30.5 & 7.2 & 4403.8 & 102.8 & 112.2 & & & & & & \\
\hline $\begin{array}{l}\text { A17 } \\
\text { Rubber Material }\end{array}$ & $\left\{\begin{array}{l}30 \text { gal drum } \\
55 \text { gal drum } \\
\text { FRP box } \\
\text { Culvert } \\
\text { other }\end{array}\right.$ & 15.5 & 17.1 & 19990.0 & & 122.1 & & & & 1.0 & 0.0 & 0.1 \\
\hline Subtotal: & & 15.5 & 17.1 & 19990.0 & & 122.1 & & & & & & \\
\hline
\end{tabular}




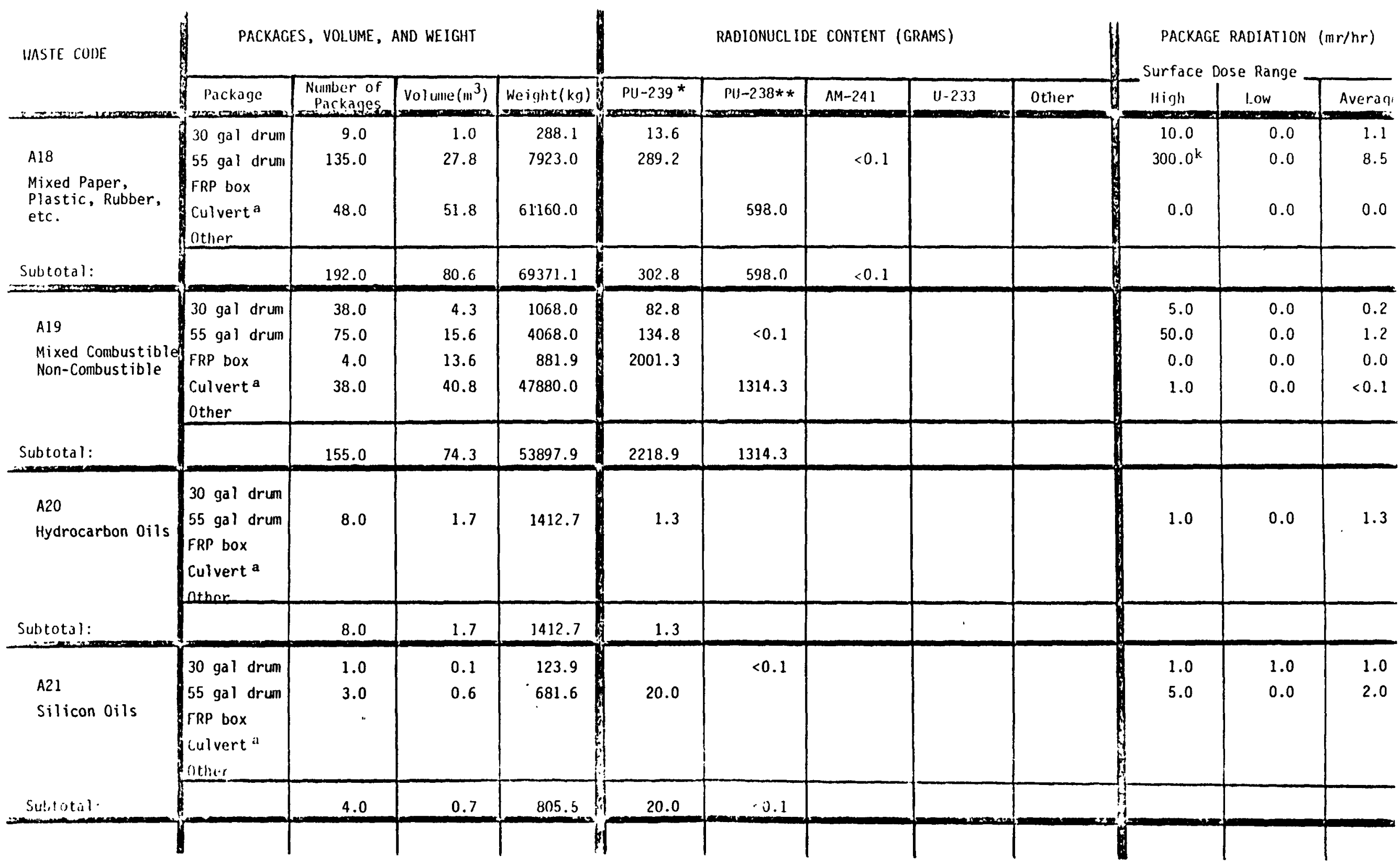




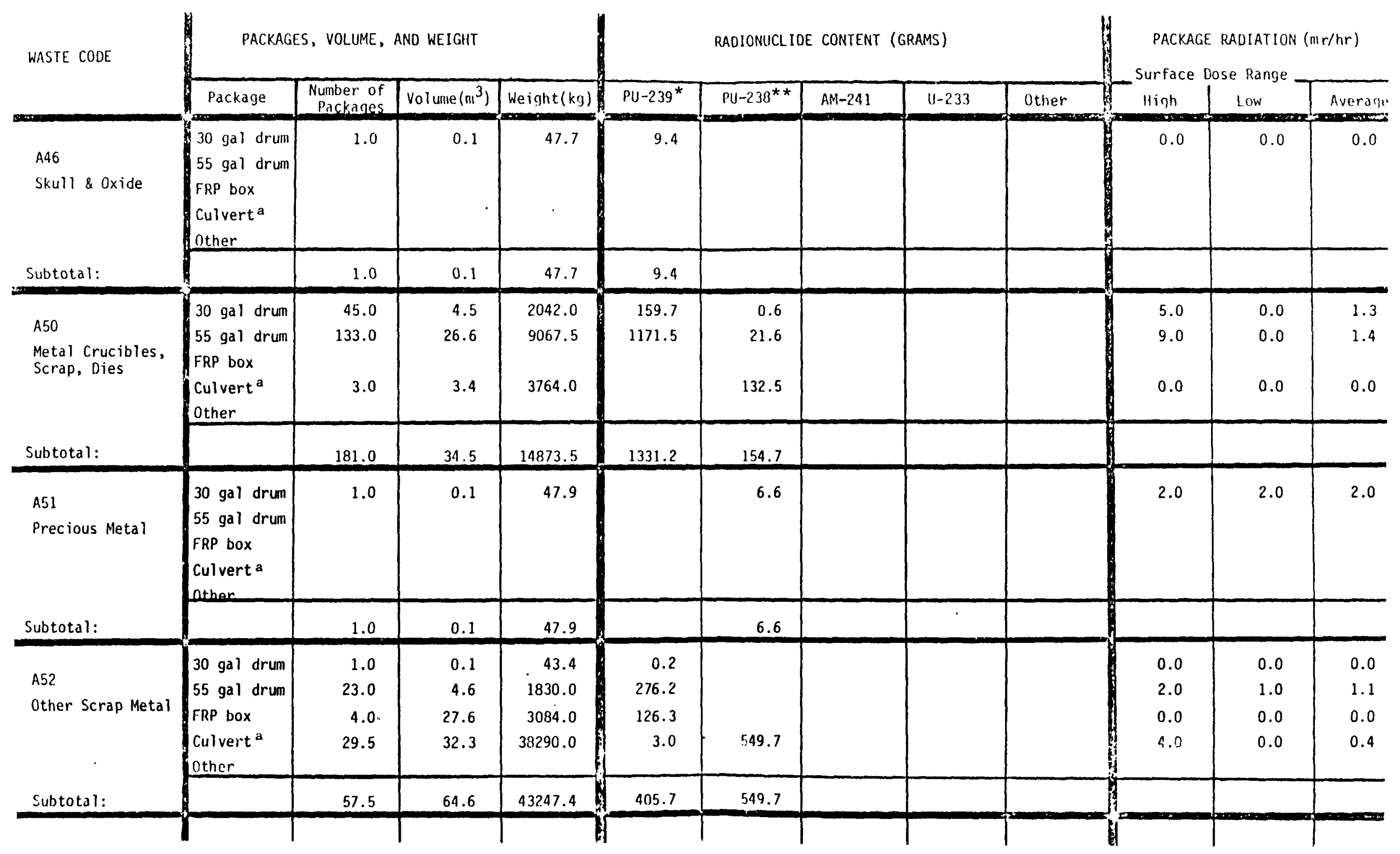




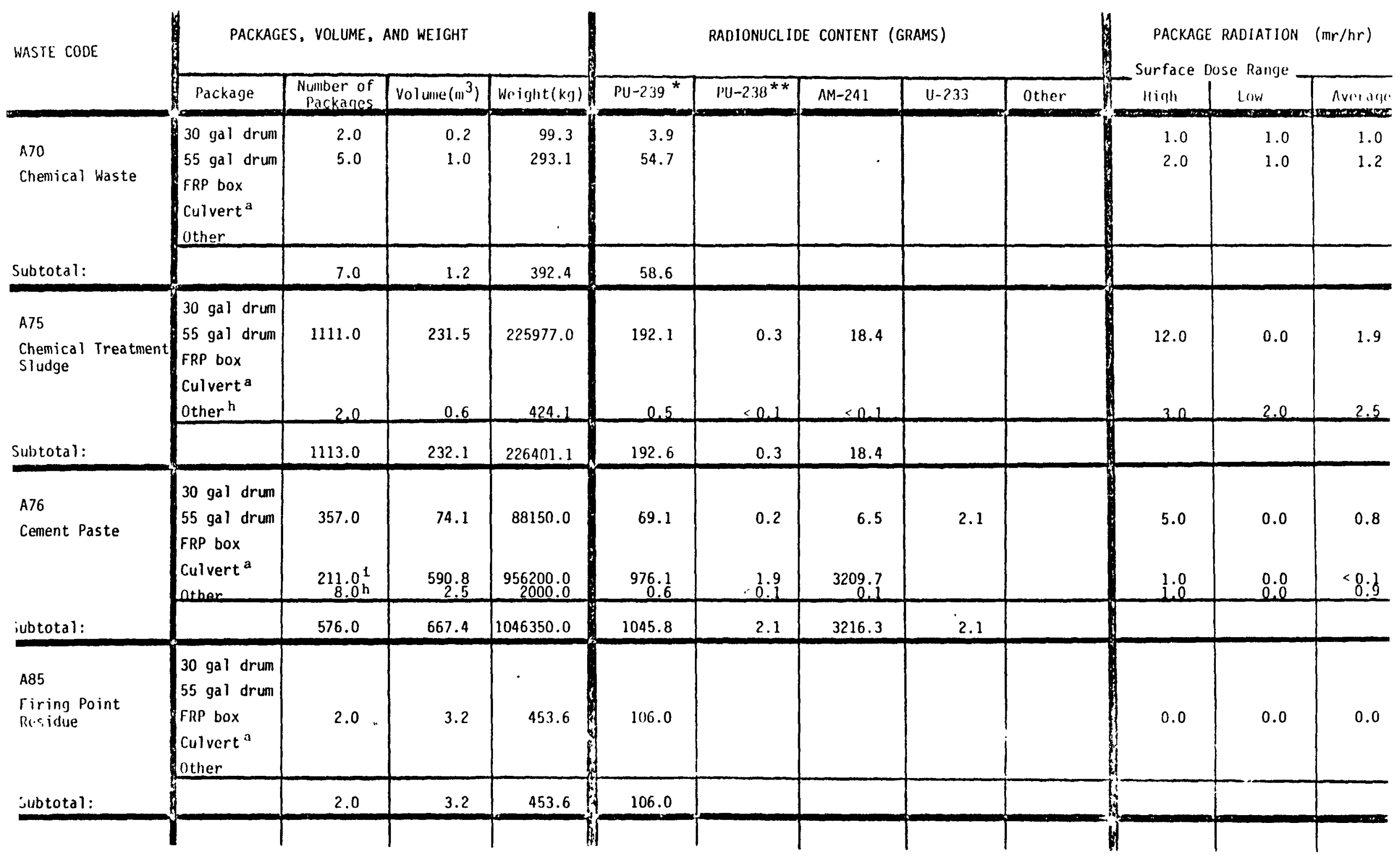




\begin{tabular}{|c|c|c|c|c|c|c|c|c|c|c|c|c|}
\hline \multirow{2}{*}{ LASTE CODE } & \multicolumn{4}{|c|}{ PACKAGES, VOLUAE, MD MEIGHT } & \multicolumn{5}{|c|}{ MADIONUCLIDE CONTENT (GRANS) } & \multicolumn{3}{|c|}{ PACKAGE RADIATION (mr/hr) } \\
\hline & Package & $\begin{array}{l}\text { Number of } \\
\text { Packanes }\end{array}$ & Volume $\left(m^{3}\right)$ & Weight $(\mathrm{kg})$ & PU-239 & $P U-238^{* \hbar}$ & $A M-241$ & U-233 & Other & High & Low & Averon \\
\hline $\begin{array}{l}\text { A90 } \\
\text { Contaminated } \\
\text { Soil }\end{array}$ & $\begin{array}{l}30 \text { gal drum } \\
55 \text { gal drum } \\
\text { FRP box } \\
\text { Culvert a } \\
\text { Other }\end{array}$ & $\begin{array}{l}46.0 \\
27.0\end{array}$ & $\begin{array}{r}9.6 \\
69.4\end{array}$ & $\begin{array}{r}8610.0 \\
42910.5\end{array}$ & $\begin{array}{r}0.6 \\
98.3\end{array}$ & & & & & $\begin{array}{l}0.0 \\
0.0\end{array}$ & $\begin{array}{l}0.0 \\
0.0\end{array}$ & $\begin{array}{l}0.0 \\
0.0\end{array}$ \\
\hline ubtotal: & & 73.0 & 79.0 & 51520.5 & 98.9 & & & & & & & \\
\hline $\begin{array}{l}\text { A95 } \\
\text { Glass }\end{array}$ & $\begin{array}{l}30 \text { gal drum } \\
55 \text { gal drum } \\
\text { FRP box } \\
\text { Culvert a } \\
\text { other }\end{array}$ & $\begin{array}{r}3.0 \\
11.0 \\
1.0\end{array}$ & $\begin{array}{l}0.3 \\
2.2 \\
1.1\end{array}$ & $\begin{array}{r}88.5 \\
669.5 \\
1342.0\end{array}$ & $\begin{array}{r}1.7 \\
52.0\end{array}$ & $\begin{array}{l}0.1 \\
6.9\end{array}$ & 0.2 & & & $\begin{array}{l}1.0 \\
2.0 \\
0.0\end{array}$ & $\begin{array}{l}0.0 \\
0.0 \\
0.0\end{array}$ & $\begin{array}{l}0.3 \\
1.0 \\
0.0\end{array}$ \\
\hline ubtotal: & & 15.0 & 3.6 & 2100.0 & 53.7 & 7.0 & 0.2 & & & & & \\
\hline $\begin{array}{l}\text { A99 } \\
\text { Unidentified } \mathrm{J} \\
\text { Material }\end{array}$ & $\begin{array}{l}30 \text { gal drum } \\
55 \text { gal drum } \\
\text { FRP box } \\
\text { Culvert a } \\
\text { Othor }\end{array}$ & $\begin{array}{l}2.0 \\
5.0 \\
1.0\end{array}$ & $\begin{array}{l}0.2 \\
1.0 \\
3.6\end{array}$ & $\begin{array}{r}77.2 \\
248.5 \\
332.3\end{array}$ & $\begin{array}{l}0.3 \\
0.2\end{array}$ & & & & 0.3 & $\begin{array}{l}1.0 \\
1.0 \\
0.0\end{array}$ & $\begin{array}{l}0.0 \\
0.0 \\
0.0\end{array}$ & $\begin{array}{l}0.5 \\
0.2 \\
0.0\end{array}$ \\
\hline ubtotal: & & 8.0 & 4.8 & 658.0 & 0.5 & & & & 0.3 & & & \\
\hline OTALS: & & 6570.5 & 4348.5 & 2206754.5 & 67762.4 & 6337.3 & 3528.7 & 4305.1 & 5.3 & & & \\
\hline
\end{tabular}

* PU - 239 : Total grams of material types 51-57. (NU - SWG).

** PU - 238 : Heat source grade material (i.e. $80 \%$ Pu-238) 
a. The culverts are concrete culvert-like casks placed vertically in the ground, each containing two 30 gallon black steel drums of waste. The volumes listed include the cask, and have been entered in the data base that way. The weights are for the 30 gallon drum, the waste inside the drum, and the culvert. The package radiation is the dose rate of the drums and not the cask, as it is impossible to measure cask dose rates since they already are partially buried when the drums are emplaced. The original intent in the use of these casks was that they became the retrievable container that would be sent to a repository. Based upon the current WIPP-WAC, it now may be desirable to devise a means of "retrieving" the stored 30 gallon drums from the culverts.

b. One package has surface dose rate at $1500 \mathrm{mr} / \mathrm{hr}$; the next highest dose rate for a package is $70 \mathrm{mr} / \mathrm{hr}$.

c. Three metal drum type containers measuring 4 feet in diameter by 6 feet high and two metal drum type containers measuring 3 feet in diameter by 6 feet high.

d. Same type drum as in "c", measuring 4 feet in diameter by 6 feet high.

e. The "other" radionuclide is NP-237.

f. The "other" radionuclide is $A C-227$.

9. The "other" radionuclides include 3.0 grams NP-237, 031 grams $\mathrm{CM}-242$, and trace amounts of BK-249, CF-249, and CM-244.

h. Containers are 83 gallon steel overpack drums measuring .95 meter in length and .7 meter in diameter.

i. Corrugated Metal Pipe containers measuring E:l reters in leng:n by .76 meter in diameter.

j. The material in the category was from very early disposals where the records were not complete.

k. One drum has a listed dose rate of $300 \mathrm{mr} / \mathrm{hr}$; the next highest is $120 \mathrm{mr} / \mathrm{hr}$.

1. Two drums have a listed dose rate of $250 \mathrm{mr} / \mathrm{hr}$; the next highest is $100 \mathrm{mr} / \mathrm{hr}$. 
SUMmARY

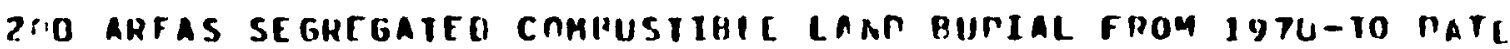
CPO GADIOACIJVF BUDIAL GPOUHOS, INCLUDING V7 IPENCH

WLPIAL CROIND

PI:O IAST AREA

OGY WASIE NO. 12B

IRANSLRAHIC

IUTAL 2OD-E

$\bullet 1$

$(v-233,5.6 \mathrm{gl} \cdot 01)$
SIIRFACE

AIRLA

volum

IsQUARI FTI ICUBIC FII

UPAN IUM

(GRAMS)

PLTONIUH (GRAMC)

$1.12 E+02$

$1.12 E+0.2$

$5.6 \mathrm{~nL}+01$

DTHER RAOTOACTIVITY (TIKAMS)

\section{$2.285+04 \quad 2.27 L \cdot 04$ \\ $2 \cdot 271 \div 04$ \\ $2 \cdot 28 E * 64$}

1 
SUMMAHY

2:II AHFAS SECRECATEL COABUSTIGIE LANR QUTIAL FROM 1970-TO MATE

CPU HALIOACTIVE BURIAL GROUNDS, INCLIDINC VT IRENCH

$$
\text { (CON IINUEO) }
$$

BUIIIAL LHUINN

SIIRFACI

200 WEST ARLA

AREA VOLUAF URANIUH

PLUTONIUH

IGRAHSI

OTHER RADIOACTIVITY

(SOUARL FT) ICUBIC FTI IGRAMSI

(GRAKS)

IIH. 7.77E+(12) IAH $-237.1 .00 E-015)$

(IH. 3.64[. I4)(U-233, $1.78 E+02)(A H-241,6.13 t-U 1)(P U-238.2 .99 E-01)$

$(\mathrm{TH}, 2.40 \mathrm{~T}+04)(\mathrm{LI}, 2 \cdot 00 \mathrm{D}+03)$

(TH, $0.86 E+f i 3)(N P-237.2 .06 E+01)(P U-238.8 .99[-01)(U-233.2 .14 E+01)$

(IH, 6.8GF+03)(U-233, 1.18E+U1)(CM-245, 2.UOE 00$)(A M-241,8.5 n E+00)$ 
LANU GURIAL OF SOLIO FAUIOACTIVL WASTES FHOH $1 / 1$ IO $12 / 31$ 1980

PIIRIAL LROURO T4C, AREA 200-4, WASTE IYPF - IRANSURANIC

TRINCII 1, PFFIOD 01/01/8ח TO 12/31/80 (N-40190 TO N-4T190), (N-77480 TO W-77583) TRENCH 4, PLRIOD 04/01/8R TO 12/31/8O

$(N-4007010 N-40470):(N-7764010 W-77721)$

\begin{tabular}{|c|c|c|c|c|c|c|c|c|c|}
\hline SHIPPER & TYPL & $\begin{array}{l}\text { YOLUML } \\
\text { CU.FT }\end{array}$ & $\begin{array}{l}\text { PU } \\
\text { GRAMS }\end{array}$ & $\begin{array}{l}\text { TOIAL } \\
\text { CURIFS }\end{array}$ & $\begin{array}{l}S R-90 \\
\text { CUR IES }\end{array}$ & $\begin{array}{l}\text { RU-IDE } \\
\text { CURIES }\end{array}$ & $\begin{array}{l}\text { CS-1 } \\
\text { CURIE }\end{array}$ & $\begin{array}{l}\text { URANIUM } \\
\text { GRAMS }\end{array}$ & $\begin{array}{l}\text { OTHEF } \\
\text { CURICS }\end{array}$ \\
\hline
\end{tabular}

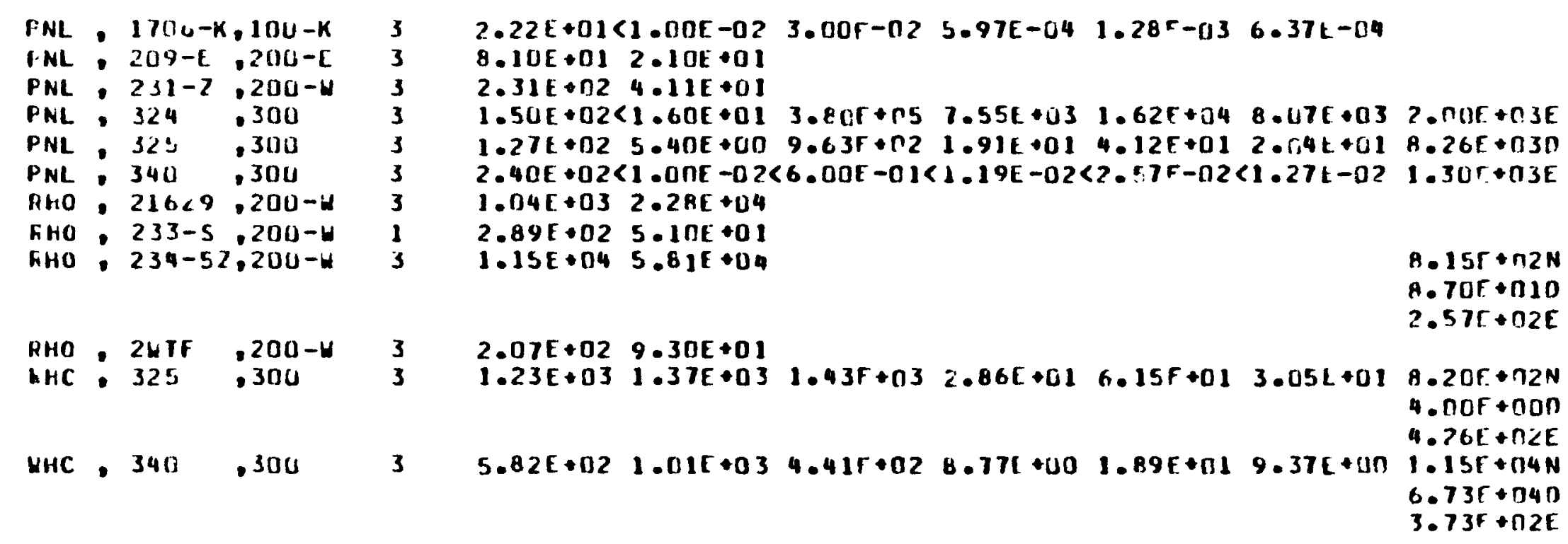

JOIAL( $1.22-001$ ACRES) $1.57 E+048.35 E+043.82 E+05 \quad 7.61(+431.64 F+048.13 L+030.32 F+04$

+1
$* 2$
+3
44

$(P U-238,3.50 E+0,1 T H, 3.00 E+00)$

$(P U-238,5.23 E+03)(T H, 2.00 E+00)$

(IH, B.EOE +01$)$

(IH, $1.30 E+C, 3)(U-233,2 \cdot 40 E-011$

* IYPE SEE APPENOIX-A 
AIPENUIX-A

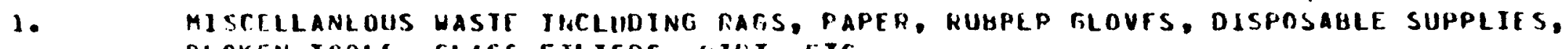
BKCKEH TOOLS, GLASS FILTERS, UIHT, ETC.

FAILEU COUIPMLNT ANU CBSOLFIE EGUIPAFNT OR MATFRIALS INCLUDING IANKS. PUMPS, AGITATORS, OVFNS, HEATERS, HONOS, JUAPERS, AND ACCLSORIES.

3. COMBINATIONS OF TYPE I AND TYPE?

4. HAOIOACTIVE ELEMENTS 
TABLE 4

Current Trends in the Generation of 239 Pu Contaminated Solid Waste Quantity ( $\mathrm{Ci})$, Volume ( $\mathrm{ft}^{3}$ ) and Concentration ( $\mathrm{Ci} / \mathrm{ft}^{3}$ )

as a Function of Time Period (All Facilities)

Data Based on COBRA* Records

\begin{tabular}{|c|c|c|c|c|c|c|}
\hline \multirow{2}{*}{$\begin{array}{l}\text { Time } \\
\text { Period }\end{array}$} & \multicolumn{3}{|c|}{$\begin{array}{l}\text { Report Code } 24 * \star \\
239 \text { pu in Culverts } \\
\end{array}$} & \multicolumn{3}{|c|}{$\begin{array}{l}\text { Report Code } 25 t \\
239_{\text {Pu in Combustibles }}\end{array}$} \\
\hline & $\mathrm{Ci}$ & $\mathrm{ft} \mathrm{t}^{3}$ & $\mathrm{Ci} / \mathrm{ft}^{3}$ & $\mathrm{Ci}$ & $\mathrm{ft}^{3}$ & $\mathrm{Ci} / \mathrm{ft}{ }^{3}$ \\
\hline 1975 & 146 & 834 & 1.17 & 121 & 23946 & 0.0050 \\
\hline 1976 & 173 & 819 & 0.21 & 179 & 21309 & 0.0084 \\
\hline 1977 & 262 & 865 & 0.30 & 260 & 26220 & 0.0099 \\
\hline 1978 & 278 & 1032 & 0.27 & 276 & 34495 & 0.0080 \\
\hline 1979 & 170 & 945 & 0.18 & 181 & 37194 & 0.0049 \\
\hline $1980+t$ & 452 & 1432 & 0.32 & 448 & 63216 & 0.0071 \\
\hline
\end{tabular}

* COBRA-Computerized Radioactive Waste Burial Records Analysis System. Values are as buried and are not decayed.

** COBRA Report Code 24, "Variety of Contamination vs. Burial Code." Includes combustibles and noncombustibles.

+ COBRA Report Code 25, "Variety of Contamination vs. Combustion Code." Includes combustibles in drums in culverts and drums on pads.

$\dagger+$ Estimated projections based on data from January-March 1980. 
TABLE 3

Current Trends in the Generation of ${ }^{236^{3}}$ Pu Contaminated Solid Haste Quantity ( $\mathrm{Ci})$, Volume $\left(\mathrm{ft}^{3}\right)$ and Concentration $\left(\mathrm{Ci} / \mathrm{ft}^{3}\right.$ )

as a Punction of Time Period (All Pacilities)

Data Based on COBRA* Records

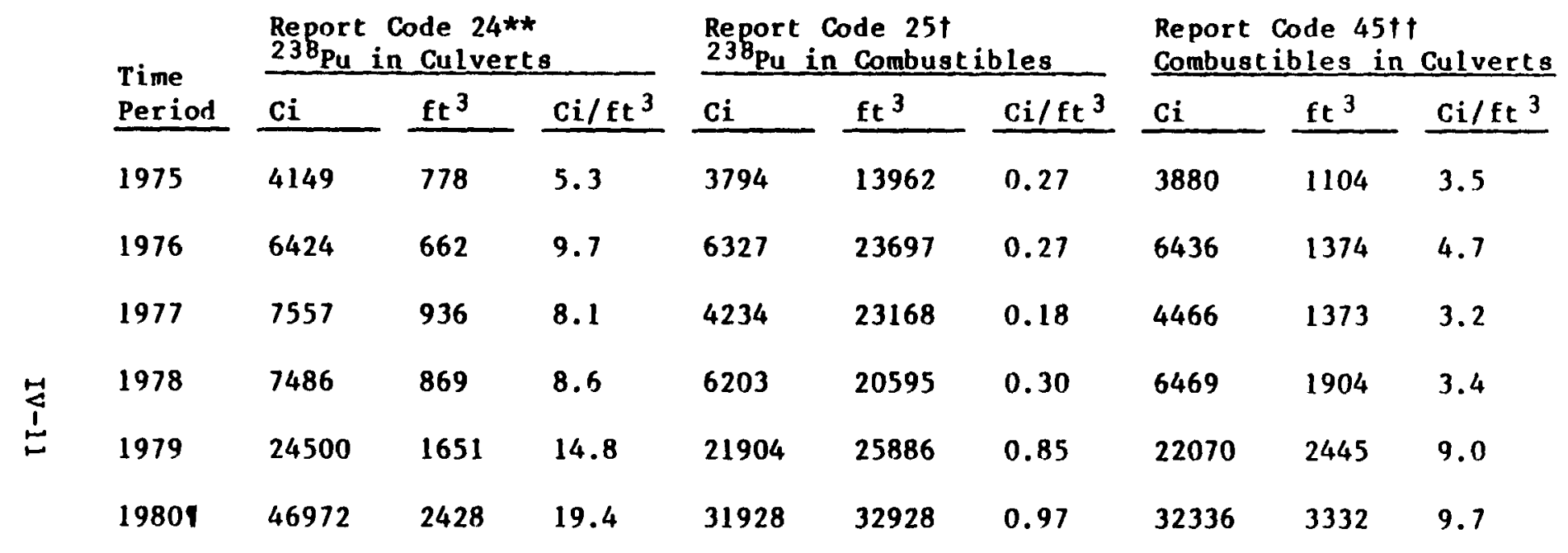

* COBRA-Computerized Radioactive Waste Burial Records Arialysis System. Values are as buried and are not decayed.

** COBRA Report Code 24, "Variety of Contamination vs. Burial Code." Includes combustibles and noncombustibles.

$\uparrow$ COBRA Report Code 25, "Variety of Contamination vs. Combustion Code." Includes combustibles in drums in culverts and drums on pads.

tt COBRA Report Code 45, "Burial Code v8. Combustion Code." Includes all TRU isotopes.

I Estimated projections based on data from January-March 1980 . 


\section{APPENDIX C \\ STORAGE SITE ENVIRONMENTAL IMPACT REVIEWS}

Environmental and Other Evaluations of Alternatives for Long-Term Management of Buried INEL Transuranic Waste, ID0-10085, U.S. Department of Energy, Idaho Operations Office, Idaho Falls, ID, December 1979.

Environmental and Other Evaluations of Alternatives for Long-Term Management of Stored INEL Transuranic Waste, D0E/ET-0081 (Revised), U.S. Department of Energy, Assistant Secretary for Energy Technology, Office of Nuclear Waste Management, Washington, D.C., December 1979.

Assessment of Alternatives for Management of ORNL Retrievable Transuranic Waste, ORNL/Sub-79/13837/5, Oak Ridge National Laboratory, Oak Ridge, TN, October 1980.

Final Environmental Impact Statement, Long-Term Management of Defense High-Level Radioactive Wastes (Research and Development Program for Immobilization, Savannah River Plant, Aiken, South Carolina, DOETEIS-0023, U.S. Department of Energy, Ass istant Secretary for Nuclear Energy, Washington, D.C., November 1979.

Final Environmental Impact Statement, Waste Management Operations, Idaho National Engineering Laboratory, Idaho, ERDA-1536, U.S. Energy Research \& Development Administration, Washington, D.C., September 1977.

Final Environmental Statement, Waste Management Operations, Hanford Reservation, Richland, Washington, ERDA-1538, U.S. Energy Research Q Development Administration, Washington, D.C., December 1975.

Alternatives for Long-Term Management of Defense Transuranic Waste at the Savannah River Plant, Aiken, South Carolina, DOETSR-WM-79-1, U.S. Department of Energy, July 1979.

Technical Status Report on Environmental Aspects of Long-Term Management of High-Level Defense Waste at the Hanford Site, RHO-LD-139, Rockwell International, Richland, WA, October 1980.

Technical Aspects of Long-Term Management Alternatives for High-Level Defense Waste at the Hanford Site, RHO-LD-141, Rockwell International, Richland, WA, October 1980.

Environmental Impact Statement of the Long-Term Management of Defense Transuranic Waste at the Idaho National Engineering Laboratory (Oraft), U.S. Department of Energy, Idaho Operations Office, Idaho Falls, ID, September 1981. 
Final Environmental Impact Statement, Waste Isolation Pilot Plant, DOE/EIS-0026, U.S. Department of Energy, Assistant Secretary of Defense Programs, Washington, D.C., October 1980.

Alternatives for Long-Term Management of Defense Transuranic/Low-Level Waste at the Hanford Site, Richland, Washington, (Draft Report), FBDU-273-01, Ford, Bacon \& Davís Utah, Inc., Salt Lake City, UT, January 1980.

Alternative TRU Waste Management Strategies at Los Alamos National Laboratory, LA-8982-MS, Los ATamos National Laboratory, Los A 1 amos, NM, September 1981.

Alternatives for Long-Term Management of Defense Transuranic Waste at the Idaho National Engineering Laboratory, ID0-10075, U.S. Department of Energy, Idaho Operations Office, Idaho Falls, ID, October 1978.

Supplemental Alternatives for Long-Term Management of Defense Transuranic Waste at the Savannah River Plant, Aiken, South Carolina, DOE/SR-WM-79-1 Supplement, U.S. Department of Energy, August 1980. 